\title{
Um problema de extensão relacionado a raiz quadrada do Laplaciano com condição de fronteira de Neumann
}

\author{
Michele de Oliveira Alves \\ TESE APRESENTADA \\ AO \\ Instituto de Matemática e Estatística \\ DA \\ Universidade DE SÃo PAUlo \\ PARA \\ OBTENÇÃO DO TÍTULO \\ $\mathrm{DE}$ \\ Doutor em Matemática Aplicada \\ Programa: Matemática Aplicada \\ Orientador: Prof. Dr. Sérgio Muniz Oliva Filho
}

Durante o desenvolvimento deste trabalho o autor recebeu auxílio financeiro da CAPES

São Paulo, janeiro de 2010 


\section{Um problema de extensão relacionado a raiz quadrada do Laplaciano com condição de fronteira de Neumann}

Esta versão definitiva da tese contém as correções e alterações sugeridas pela Comissão Julgadora durante a defesa realizada por Michele de Oliveira Alves em 15/12/2010.

Comissão Julgadora:

- Prof. Dr. Sérgio Muniz Oliva Filho (orientador) - IME-USP

- Prof. Dr. Orlando Francisco Lopes - IME-USP

- Prof. Dr. Plácido Zoega Táboas - ICMC-USP

- Prof. Dr. Sérgio Henrique Monari Soares - ICMC-USP

- Profa. Dra. Joana Isabel Afonso Mourão Terra - UFBA 
Dedico à minha família. 


\section{Agradecimentos}

Ao final deste trabalho quero primeiramente agradecer à Deus por acreditar em mim e por me dar a graça de concluir mais uma etapa de minha vida. Pelo apoio e sustento sempre concedido nos momentos mais difíceis.

À minha família por ser minha base, dando-me apoio, alegria, amor e por compreender minha ausência em muitos momentos. Ela com certeza é parte fundamental desta conquista.

Ao meu noivo Emerson pela paciência, amor e carinho, pelas inúmeras vezes que me incentivou a continuar e a acreditar mais em mim. Obrigada pela sua presença, ela foi meu combustível para prosseguir sempre.

Ao professor Sérgio por todos os ensinamentos, paciência, amizade, disponibilidade para me atender, esclarecer dúvidas, etc... com ele obtive um grande crescimento matemático.

Aos meus amigos de pós-graduação pela amizade. Em particular, Fernanda e Anderson (Super!) pela maravilhosa convivência não só no IME mas também em casa. Vocês com certeza acompanharam de perto toda esta minha trajetória. Obrigada pelas conversas e inúmeras risadas que tivemos ao longo deste período.

À minha amiga Gleiciane pelo convívio iniciado na graduação, pelos estudos durante o período de disciplinas, seminários e por várias vezes sanar minhas dúvidas.

Agradeço também aos amigos da Paróquia Nossa Senhora dos Pobres que me acolheram com tanto amor e carinho.

Aos professores do departamento e funcionários do IME. Também aos professores Orlando Francisco Lopes, Plácido Zoega Táboas, Sérgio Henrique Monari Soares e Joana Isabel Afonso Mourão Terra pelas correções e importantes sugestões.

À CAPES pelo auxílio financeiro. 


\section{Resumo}

Neste trabalho definimos o operador não local, raiz quadrada do Laplaciano com condição de fronteira de Neumann, através do método de extensão harmônica. O estudo foi feito com o auxílio das séries de Fourier em domínios limitados, como sendo o intervalo, o quadrado e a bola. Posteriormente, aplicamos nosso estudo, à problemas elípticos não lineares envolvendo o operador não local raiz quadrada do Laplaciano com condição de fronteira de Neumann.

Palavras-chave: raiz quadrada do Laplaciano, condições de fronteira de Neumann, operador não local, problemas não lineares, extensão harmônica, série de Fourier. 


\begin{abstract}
In this work we define the non-local operator, square root of the Laplacian with Neumann boundary condition, using the method of harmonic extension. The study was done with the aid of Fourier series in bounded domains, as the interval, the square and the ball. Subsequently, we apply our study, the nonlinear elliptic problems involving non-local operator square root of the Laplacian with Neumann boundary condition.
\end{abstract}

Keywords: square root of the Laplacian, Neumann boundary conditions, non-local operator, nonlinear problems, harmonic extension, Fourier series. 


\section{Sumário}

Lista de Símbolos $\quad$ xi

1 Introdução 1

2 Preliminares $\quad 9$

2.1 Espaços de Sobolev, Slobodeckij e Hölder . . . . . . . . . . . . . . . . . . . . 10

2.1 .1 Imersões Contínuas e Compactas . . . . . . . . . . . . . . . . . . . . 12

2.1.2 Teorema do Traço e Desigualdade de Poincaré . . . . . . . . . . . . . . . . 13

2.2 Espaços de Sobolev das funções periódicas . . . . . . . . . . . . . . . . . . . 15

2.2.1 Teorema do Traço para as funções periódicas . . . . . . . . . . . . . . . 19

3 Cálculo Funcional $\quad 21$

3.1 Estudo do Operador $-\Delta_{N} \ldots \ldots \ldots \ldots \ldots \ldots \ldots \ldots \ldots \ldots$

3.2 Raiz Quadrada do operador $-\Delta_{N} \ldots \ldots \ldots \ldots \ldots \ldots$

4 O Operador $\quad 35$

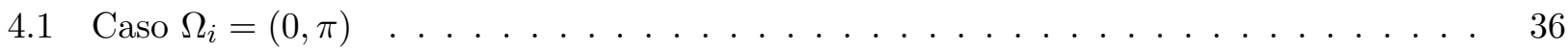

4.2 Caso $\Omega_{q}=(0, \pi) \times(0, \pi) \ldots \ldots \ldots \ldots \ldots \ldots \ldots \ldots$

4.3 Caso $\Omega_{b}=B(0, \pi) \ldots \ldots \ldots \ldots \ldots \ldots \ldots \ldots$

5 Aplicação $\quad 81$

5.0 .1 Solução fraca no intervalo . . . . . . . . . . . . . . . . . . 82

5.0 .2 Solução fraca no quadrado . . . . . . . . . . . . . . . . 84

5.0 .3 Existência de Solução Fraca . . . . . . . . . . . . . . . . . . . . 86

$\begin{array}{lr}\text { A Apêndice } & 99\end{array}$

A.1 Funções Especiais . . . . . . . . . . . . . . . . . . . . . . . . . . . . . . . . . . 99

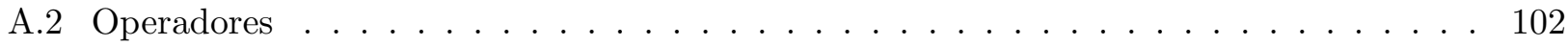

A.3 Teorema do Multiplicador de Lagrange . . . . . . . . . . . . . . . . . . . . 103

$\begin{array}{lr}\text { Referências Bibliográficas } & 105\end{array}$

$\begin{array}{lr}\text { Índice Remissivo } & 109\end{array}$ 


\section{Lista de Símbolos}

$$
\begin{aligned}
& \Delta_{N} \quad \text { Operador Laplaciano com condição de fronteira de Neumann } \\
& C^{t}\left(\mathbb{R}^{n}\right) \quad \text { Espaço de Hölder sobre } \mathbb{R}^{n} \\
& \bar{C}^{t}(U) \quad \text { Espaço de Hölder sobre } U \\
& C_{\text {per }}^{\infty}\left(\mathbb{R}^{n}\right) \quad \text { Conjunto das funções infinitamente diferenciáveis que são } 2 \pi \text {-periódicas } \\
& C_{\text {per }}^{\infty}\left(\overline{\mathbb{R}_{+}^{n+1}}\right) \quad \text { Conjunto das funções infinitamente diferenciáveis que são } 2 \pi \text {-periódicas em } x \in \mathbb{R}^{n} \\
& L^{2}(U) \quad \text { Espaço de Lebesgue em } U \\
& L^{2}(U ; r) \quad \text { Espaço de Lebesgue } L^{2}(U) \text { com peso } r \\
& H^{s}(U) \quad \text { Espaço de Sobolev se } s \in \mathbb{Z}_{+} \text {e Espaço de Slobodeckij se } s>0 \text { não inteiro } \\
& H_{p e r}^{s}\left(\mathbb{R}^{n}\right) \quad \text { Espaço de Sobolev periódico na variável } x \in \mathbb{R}^{n} \\
& H_{\text {per }}^{s}\left(\mathbb{R}^{n+1}\right) \quad \text { Espaço de Sobolev periódico na variável } x \in \mathbb{R}^{n} \text {, para todo }(x, z) \in \mathbb{R}_{+}^{n+1} \\
& J_{p} \quad \text { Função de Bessel de primeiro tipo de ordem } p \in \mathbb{R} \\
& Y_{p} \quad \text { Função de Bessel de segundo tipo de ordem } p \in \mathbb{R} \text { fracionário } \\
& \hookrightarrow \quad \text { Imersão Contínua } \\
& \stackrel{c}{\hookrightarrow} \quad \text { Imersão Compacta } \\
& \mathbb{R}_{+}^{n+1}=\left\{(x, z) \in \mathbb{R}^{n+1} \mid x \in \mathbb{R}^{n} \text { e } z>0\right\} \\
& \Omega_{i} \quad=(0, \pi) \\
& \Omega_{b} \quad=B(0, \pi) \\
& \Omega_{q}=(0, \pi) \times(0, \pi) \\
& \mathcal{I} \quad=\mathbb{N}^{*} \text { em } \Omega_{i} \\
& \mathcal{I}=(\mathbb{N} \times \mathbb{N}) \backslash\{(0,0)\} \text { em } \Omega_{q} \\
& \mathcal{I} \quad=\left(\mathbb{N}^{*} \times \mathbb{N}\right) \backslash\{(1,0)\} \text { em } \Omega_{b} \\
& X \quad=\left\{u \in L^{2}(\Omega) \mid \int_{\Omega} u(x) d x=0\right\}
\end{aligned}
$$




$$
\begin{aligned}
Q^{n} & =\left\{x \in \mathbb{R}^{n}|| x_{j} \mid \leq \pi, \forall j=1, \cdots, n\right\} \\
u_{\Omega} & =\int_{\Omega} u(x) d x \\
<u, v>\quad & \int_{\Omega} u(x) v(x) d x \\
(\tilde{v}(\cdot, \cdot, z))_{\Omega} & =\int_{\Omega} \tilde{v}(x, y, z) d x d y
\end{aligned}
$$




\section{Capítulo 1}

\section{Introdução}

As potências fracionárias do Laplaciano são geradores infinitesimais do processo de difusão estável chamado de processo de Lévy e aparecem nas dinâmicas populacionais, reações químicas em líquidos, entre outros assuntos do cotidiano.

Neste trabalho, estudamos a raiz quadrada do operador Laplaciano com condição de fronteira de Neumann, $\left(-\Delta_{N}\right)^{\frac{1}{2}}$, em três tipos de domínios, utilizando técnicas de extensão harmônica. Posteriormente, aplicamos à problemas elípticos não lineares.

As potências fracionárias do operador Laplaciano, podem ser interpretadas de algumas formas.

Considere uma função $u: \mathbb{R}^{n} \longrightarrow \mathbb{R}$ suficientemente regular. Utilizando a Transformada de Fourier, temos

$$
\widehat{(-\Delta)^{s}} u(\xi)=|\xi|^{2 s} \widehat{u}(\xi)
$$

e ainda, através da Fórmula da Derivada Fracionária de Riesz,

$$
(-\Delta)^{s} u(x)=C_{n, s} \int_{\mathbb{R}^{n}} \frac{u(x)-u(y)}{|x-y|^{n+2 s}} d y
$$

onde $C_{n, s}$ é alguma constante de normalização e $s \in(0,1)$.

As potências negativas são dadas por

$$
(-\Delta)^{\alpha}=\frac{1}{2 \pi i} \int_{\gamma}(-\lambda)^{\alpha}(\lambda I-\Delta)^{-1} d \lambda
$$

onde $\gamma$ é qualquer curva simples suave por partes satisfazendo algumas propriedades e $\operatorname{Re}(\alpha)<0$.

Para $s=\frac{1}{2}$, podemos ainda considerar $(-\Delta)^{\frac{1}{2}}$ como sendo um operador positivo tal que

$$
(-\Delta)^{\frac{1}{2}} \circ(-\Delta)^{\frac{1}{2}} u=-\Delta u
$$

para qualquer $u \in D(-\Delta u)$.

O método de definir o operador $(-\Delta)^{\frac{1}{2}}$ por meio de técnicas extensão harmônica, consiste em considerar o operador $T$ dado por

$$
u \longmapsto T(u)(x)=-v_{z}(x, 0),
$$


onde $u: \mathbb{R}^{n} \longrightarrow \mathbb{R}$ é uma função suave limitada e $v: \mathbb{R}_{+}^{n+1} \longrightarrow \mathbb{R}$ é a única solução do problema

$$
\left\{\begin{array}{ccccl}
\Delta v(x, z) & = & 0 & \text { em } & \mathbb{R}_{+}^{n+1}=\mathbb{R}^{n} \times(0, \infty) \\
v(x, 0) & = & u(x) & \text { em } & \mathbb{R}^{n}
\end{array} .\right.
$$

Observe que

1. Se colocarmos $-v_{z}(x, 0)$ como condição de Dirichlet no problema (1.1), verificamos que $-v_{z}(x, z)$ é solução deste novo problema, logo pela definição do operador $T$, temos que

$$
T(T(u))(x)=T\left(-v_{z}(x, 0)\right)=v_{z z}(x, 0)=-\Delta_{x} v(x, 0),
$$

Então,

$$
(T \circ T)(u))(x)=-\Delta_{x} u(x) .
$$

2. Usando o Método de Integração por Partes, vemos que

$$
\begin{aligned}
\int_{\mathbb{R}^{n}} u(x) T(u)(x) d x & =-\int_{\mathbb{R}^{n}} v(x, 0) v_{z}(x, 0) d x \\
& =-\int_{\mathbb{R}_{+}^{n+1}} v(x, z) \Delta v(x, z) d x d z+\int_{\mathbb{R}_{+}^{n+1}}|\nabla v(x, z)|^{2} d x d z \\
& =\int_{\mathbb{R}_{+}^{n+1}}|\nabla v(x, z)|^{2} d x d z \geq 0,
\end{aligned}
$$

logo, o operador $T$ é positivo.

Portanto, o operador $T$ que mapeia a condição de Dirichlet $u$ na condição de Neumann $-v_{z}(\cdot, 0)$ é de fato o operador $(-\Delta)^{\frac{1}{2}}$.

O artigo [8] generalizou este método com o objetivo de definir um problema de extensão semelhante para cada potência fracionária do Laplaciano. Para uma função $u: \mathbb{R}^{n} \longrightarrow \mathbb{R}$ suave limitada, considerou-se o seguinte problema

$$
\left\{\begin{array}{ccccc}
\Delta_{x} v(x, z)+\frac{a}{z} v_{z}(x, z)+v_{z z}(x, z) & = & 0 & \text { em } & \mathbb{R}_{+}^{n+1} \\
v(x, 0) & = & u(x) & \text { em } & \mathbb{R}^{n}
\end{array},\right.
$$

tal que $a=1-2 s$.

Com isso, mostrou-se que

$$
(-\Delta)^{s} u(x)=-v_{z}(x, 0)
$$

onde $s \in(0,1)$ e a potência fracionária do operador Laplaciano foi expressada pela Fórmula da Derivada Fracionária de Riesz.

Outro trabalho baseado nesta idéia de extensão harmônica é o artigo de [7], onde estudou-se a raiz quadrada do operador Laplaciano com condição de fronteira de Dirichlet $\left(-\Delta_{D}\right)^{\frac{1}{2}}$, em um domínio $\Omega \subset \mathbb{R}^{n}$ limitado, suave. Tal operador foi definido via método de extensão harmônica no cilindro $\mathcal{C}=\Omega \times(0, \infty)$. 
Por meio de algumas pesquisas, observamos que não se têm na literatura, um estudo das potências fracionárias do operador Laplaciano com condição de fronteira de Neumann, via método de extensão harmônica e ainda usando séries de Fourier.

Nosso trabalho foi definir a raiz quadrada do operador Laplaciano com condição de fronteira de Neumann $\left(-\Delta_{N}\right)^{\frac{1}{2}}$, usando o método de extensão harmônica e o auxílio das séries de Fourier em três tipos de domínios, o intervalo $\Omega_{i}=(0, \pi)$, o quadrado $\Omega_{q}=(0, \pi) \times(0, \pi)$ e bola $\Omega_{b}=B(0, \pi)$.

Para definir o operador $\left(-\Delta_{N}\right)^{\frac{1}{2}}$, consideramos os seguintes problemas de extensão

$$
\left\{\begin{array}{llll}
\Delta \tilde{v}(x, y, z) & =0 & \text { em } & \mathbb{R}_{+}^{3} \\
\tilde{v}(x, y, 0) & =\tilde{u}(x, y) & \text { em } \quad \mathbb{R}^{2} \\
\lim _{z \rightarrow \infty}\|\tilde{v}(\cdot, \cdot, z)\|_{L^{2}(\Omega)} & =0 & & \\
\lim _{z \rightarrow \infty}\left\|\tilde{v}_{z}(\cdot, \cdot, z)\right\|_{L^{2}(\Omega)} & =0 & & \\
(\tilde{v}(\cdot, \cdot, z))_{\Omega} & =0 & \forall z \geq 0
\end{array}\right.
$$

quando o domínio for $\Omega_{q}$ ou $\Omega_{b}$, onde

$$
(\tilde{v}(\cdot, \cdot, z))_{\Omega}=\int_{\Omega} \tilde{v}(x, y, z) d x d y
$$

para qualquer $z \geq 0 \mathrm{e}$

$$
\left\{\begin{array}{llll}
\Delta \tilde{v}(x, z) & =0 & \text { em } & \mathbb{R}_{+}^{2} \\
\tilde{v}(x, 0) & =\tilde{u}(x) & \text { em } \quad \mathbb{R} \\
\lim _{z \rightarrow \infty}\|\tilde{v}(\cdot, z)\|_{L^{2}\left(\Omega_{i}\right)} & =0 & \\
\lim _{z \rightarrow \infty}\left\|\tilde{v}_{z}(\cdot, z)\right\|_{L^{2}\left(\Omega_{i}\right)} & =0 & \\
(\tilde{v}(\cdot, z))_{\Omega_{i}} & =0 & \forall z \geq 0
\end{array}\right.
$$

quando o domínio for $\Omega_{i}$, onde

$$
(\tilde{v}(\cdot, z))_{\Omega_{i}}=\int_{\Omega_{i}} \tilde{v}(x, z) d x
$$

para qualquer $z \geq 0$.

O estudo foi feito com o auxílio das séries de Fourier, onde definimos o operador $\left(-\Delta_{N}\right)^{\frac{1}{2}}$ como sendo uma série das autofunções e autovalores de $-\Delta_{N}$, ou seja,

$$
\left(-\Delta_{N}\right)^{\frac{1}{2}} u=\sum_{i \in \mathcal{I}}^{\infty} \lambda_{i}^{\frac{1}{2}}<u, \varphi_{i}>\varphi_{i}
$$

onde $\varphi_{i}$ e $\lambda_{i}$ são as autofunções e os autovalores de $-\Delta_{N}$, respectivamente.

Verificamos que o operador $\left(-\Delta_{N}\right)^{\frac{1}{2}}$ é de fato a raiz quadrada do Laplaciano com condição de fronteira de Neumann, pois,

$$
\left(-\Delta_{N}\right)^{\frac{1}{2}} \circ\left(-\Delta_{N}\right)^{\frac{1}{2}} u=\sum_{i \in \mathcal{I}}^{\infty} \lambda_{i}<u, \varphi_{i}>\varphi_{i}=-\Delta_{N} u,
$$

para qualquer $u \in D\left(-\Delta_{N}\right)$.

O uso das séries de Fourier em nosso estudo foi muito importante pois tivemos a oportunidade de 
fazer todas as contas e verificar quais eram de fato as soluções dos problemas de extensão, bem como para definir o operador $\left(-\Delta_{N}\right)^{\frac{1}{2}}$.

Como uma aplicação, estudamos a existência de soluções fracas não nulas de problemas elípticos não lineares envolvendo o operador não local $\left(-\Delta_{N}\right)^{\frac{1}{2}}$ nos domínios $\Omega_{i}$ e $\Omega_{q}$. Ou seja, estudamos o seguinte problema elíptico não linear

$$
\left(-\Delta_{N}\right)^{\frac{1}{2}} u=u^{p}
$$

onde $p=2+\frac{1}{s}$, com $s>1$ ímpar, quando o domínio for $\Omega_{q}$, e $p=\bar{p}+\frac{1}{s}$, com $\bar{p}$ par e $s \geq 1$ ímpar, quando o domínio for $\Omega_{i}$.

Como sabemos, o operador $\left(-\Delta_{N}\right)^{\frac{1}{2}}$ é não local. Assim uma das vantagens de definirmos este operador por meio de técnicas de extensão harmônica é que resolver o problema elíptico não local (1.2), se resume em resolver o seguinte problema elíptico local

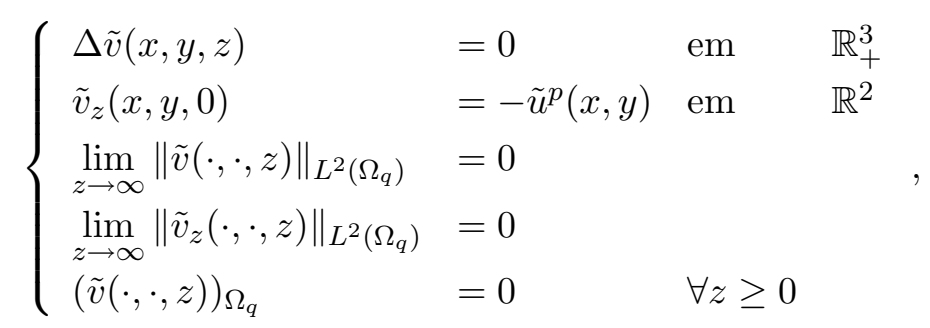

tal que $\tilde{v}$ é par, periódica nas variáveis $x$ e $y, \tilde{u}$ é a extensão par, periódica de $u$ à $\mathbb{R}^{2}$.

Quando o domínio for $\Omega_{i}$, o problema elíptico local correspondente é

$$
\left\{\begin{array}{llll}
\Delta \tilde{v}(x, z) & =0 & \text { em } & \mathbb{R}_{+}^{2} \\
\tilde{v}_{z}(x, 0) & =-\tilde{u}^{p}(x) & \text { em } \quad \mathbb{R} \\
\lim _{z \rightarrow \infty}\|\tilde{v}(\cdot, z)\|_{L^{2}\left(\Omega_{i}\right)} & =0 & & \\
\lim _{z \rightarrow \infty}\left\|\tilde{v}_{z}(\cdot, z)\right\|_{L^{2}\left(\Omega_{i}\right)} & =0 & & \\
(\tilde{v}(\cdot, z))_{\Omega_{i}} & =0 & \forall z \geq 0
\end{array},\right.
$$

onde $\tilde{v}$ é par, periódica na variável $x, \tilde{u}$ é uma extensão par, $2 \pi$-periódica de $u$ à $\mathbb{R}$.

O trabalho está organizado da seguinte maneira. No Capítulo 3, fizemos um estudo do operador local Laplaciano com condição de fronteira de Neumann,

$$
\begin{aligned}
-\Delta_{N}: D\left(-\Delta_{N}\right) \subset X & \longrightarrow X \\
u & \longmapsto-\Delta(u),
\end{aligned}
$$

onde

$$
D\left(-\Delta_{N}\right)=\left\{u \in H^{2}(\Omega) \quad\left|\frac{\partial u}{\partial n}\right|_{\partial \Omega}=0 \text { e }(u)_{\Omega}=0\right\},
$$

para verificarmos que faz sentido considerar a potência fracionária deste operador.

Com isso, usamos a teoria de Cálculo Funcional de [15] e [27], para escrevermos o operador

$$
\left(-\Delta_{N}\right)^{\frac{1}{2}}: D\left(\left(-\Delta_{N}\right)^{\frac{1}{2}}\right) \subset X \longrightarrow X
$$


como sendo uma série envolvendo as autofunções e os autovalores, do operador $-\Delta_{N}$, ou seja,

$$
\left(-\Delta_{N}\right)^{\frac{1}{2}} u=\sum_{i \in \mathcal{I}}^{\infty} \lambda_{i}^{\frac{1}{2}}<u, \varphi_{i}>\varphi_{i}
$$

onde $\varphi_{i}$ são as autofunções de $-\Delta_{N}$, nos domínios citados anteriormente, $\lambda_{i}$ são os correspondentes autovalores e

$$
D\left(\left(-\Delta_{N}\right)^{\frac{1}{2}}\right)=\left\{u \in L^{2}(\Omega) \mid(u)_{\Omega}=0 \text { e } \sum_{i \in \mathcal{I}}^{\infty} \lambda_{i}\left|<u, \varphi_{i}>\right|^{2}<\infty\right\} .
$$

No Capítulo 4, definimos o operador $A_{\frac{1}{2}}: D\left(A_{\frac{1}{2}}\right) \subset X \longrightarrow X$ tal que

$$
A_{\frac{1}{2}} u=-\left.\left(\tilde{v}_{z}(\cdot, 0)\right)\right|_{\Omega}
$$

onde

$$
D\left(A_{\frac{1}{2}}\right)=\left\{u \in H^{s}(\Omega)\left|\frac{\partial u}{\partial n}\right|_{\partial \Omega}=0 \text { e }(u)_{\Omega}=0\right\}
$$

com $s>2$ para $\Omega_{q}$ e $\Omega_{b}, s>\frac{3}{2}$ para $\Omega_{i}$, e $\tilde{v}$ é a única solução clássica de

$$
\left\{\begin{array}{llll}
\Delta \tilde{v}(x, y, z) & =0 & \text { em } & \mathbb{R}_{+}^{3} \\
\tilde{v}(x, y, 0) & =\tilde{u}(x, y) & \text { em } \quad \mathbb{R}^{2} \\
\lim _{z \rightarrow \infty}\|\tilde{v}(\cdot, \cdot, z)\|_{L^{2}(\Omega)} & =0 & & \\
\lim _{z \rightarrow \infty}\left\|\tilde{v}_{z}(\cdot, \cdot, z)\right\|_{L^{2}(\Omega)} & =0 & \\
(\tilde{v}(\cdot, \cdot, z))_{\Omega} & =0 & \forall z \geq 0
\end{array}\right.
$$

quando o domínio for $\Omega_{q}$ ou $\Omega_{b}$, ou solução clássica de

$$
\left\{\begin{array}{llll}
\Delta \tilde{v}(x, z) & =0 & \text { em } & \mathbb{R}_{+}^{2} \\
\tilde{v}(x, 0) & =\tilde{u}(x) & \text { em } & \mathbb{R} \\
\lim _{z \rightarrow \infty}\|\tilde{v}(\cdot, z)\|_{L^{2}\left(\Omega_{i}\right)} & =0 & \\
\lim _{z \rightarrow \infty}\left\|\tilde{v}_{z}(\cdot, z)\right\|_{L^{2}\left(\Omega_{i}\right)} & =0 & \\
(\tilde{v}(\cdot, z))_{\Omega_{i}} & =0 & \forall z \geq 0
\end{array},\right.
$$

quando o domínio for $\Omega_{i}$.

A partir desta definição, consideramos uma extensão de $A_{\frac{1}{2}}$ definida da seguinte maneira

$$
\begin{aligned}
B_{\frac{1}{2}}: Y \subset X & \longrightarrow X \\
u & \longmapsto \sum_{i \in \mathcal{I}}^{\infty} \lambda_{i}^{\frac{1}{2}}<u, \varphi_{i}>\varphi_{i}
\end{aligned}
$$

onde

$$
Y=\left\{u \in L^{2}(\Omega) \mid(u)_{\Omega}=0 \text { e } \sum_{i \in \mathcal{I}}^{\infty} \lambda_{i}\left|<u, \varphi_{i}>\right|^{2}<\infty\right\}
$$


Com isso, provamos o seguinte teorema.

Teorema 1. O operador $B_{\frac{1}{2}}$ coincide com o operador $\left(-\Delta_{N}\right)^{\frac{1}{2}}$, ou seja,

$$
<u, B_{\frac{1}{2}} u>\geq 0
$$

para qualquer $u \in D\left(B_{\frac{1}{2}}\right) e$

$$
B_{\frac{1}{2}} \circ B_{\frac{1}{2}} u=-\Delta_{N} u
$$

para qualquer $u \in D\left(-\Delta_{N}\right)$.

No Capítulo 5, verificamos a existência de solução fraca não nula do problema não linear (1.2), com o auxílio do Teorema do Multiplicador de Lagrange em espaços lineares topológicos para os domínios $\Omega_{i}$ e $\Omega_{q}$.

Para isto, consideramos as seguintes condições

1. Se o domínio for $\Omega_{i}$

(a) $\tilde{v}(-x, z)=\tilde{v}(x, z)$, quase sempre em $x \in \mathbb{R}$, para qualquer $z \geq 0$,

(b) $\tilde{v}(x+\pi, 0)=-\tilde{v}(x, 0)$, quase sempre em $x \in \mathbb{R}$,

(c) $(\tilde{v}(\cdot, z))_{\Omega_{i}}=0$, para qualquer $z \geq 0$.

2. Se o domínio for $\Omega_{q}$

(a) $\tilde{v}(-x, y, z)=\tilde{v}(x,-y, z)=\tilde{v}(x, y, z)$, quase sempre em $(x, y) \in \mathbb{R}^{2}$, para qualquer $z \geq 0$,

(b) $\tilde{v}(x+\pi, y, 0)=-\tilde{v}(x, y, 0)$, quase sempre em $(x, y) \in \mathbb{R}^{2}$,

(c) $(\tilde{v}(\cdot, \cdot, z))_{\Omega_{q}}=0$, para qualquer $z \geq 0$.

Sejam as aplicações

$$
\begin{aligned}
I_{i}: H_{i} & \longrightarrow \mathbb{R} \\
\tilde{v} & \longmapsto \frac{1}{2} \int_{0}^{\infty} \int_{\Omega_{i}}|\nabla \tilde{v}(x, z)|^{2} d x d z,
\end{aligned}
$$

onde

$$
H_{i}=\left\{\tilde{v} \in H_{p e r}^{1}\left(\mathbb{R}_{+}^{2}\right) \mid \tilde{v} \text { satisfaz 1. e } \lim _{T \rightarrow \infty}\|\tilde{v}(\cdot, T)\|_{L^{2}\left(\Omega_{i}\right)}=\lim _{T \rightarrow \infty}\left\|\tilde{v}_{z}(\cdot, T)\right\|_{L^{2}\left(\Omega_{i}\right)}=0\right\},
$$

e

$$
\begin{aligned}
I_{q}: H_{q} & \longrightarrow \mathbb{R} \\
\tilde{v} & \longmapsto \frac{1}{2} \int_{0}^{\infty} \int_{\Omega_{q}}|\nabla \tilde{v}(x, y, z)|^{2} d x d y d z,
\end{aligned}
$$

onde

$$
H_{q}=\left\{\tilde{v} \in H_{p e r}^{1}\left(\mathbb{R}_{+}^{3}\right) \mid \tilde{v} \text { satisfaz } 2 . \mathrm{e} \lim _{T \rightarrow \infty}\|\tilde{v}(\cdot, \cdot, T)\|_{L^{2}\left(\Omega_{q}\right)}=\lim _{T \rightarrow \infty}\left\|\tilde{v}_{z}(\cdot, \cdot, T)\right\|_{L^{2}\left(\Omega_{q}\right)}=0\right\} .
$$


Para algum $a>0$ fixo, verificamos a existência de mínimo dos funcionais $I_{i}$ e $I_{q}$, respectivamente, sobre os conjuntos

$$
\begin{aligned}
& H_{i, a}=\left\{\tilde{v} \in H_{i} \mid \int_{\Omega_{i}}(\tilde{v}(x, 0))^{p+1} d x=a\right\}, \\
& H_{q, a}=\left\{\tilde{v} \in H_{q} \mid \int_{\Omega_{q}}(\tilde{v}(x, y, 0))^{p+1} d x d y=a\right\} .
\end{aligned}
$$

Através deste mínimo, mostramos a existência de solução fraca não nula do problema não linear (1.2) nos domínios $\Omega_{i}$ e $\Omega_{q}$, utilizando o Teorema do Multiplicador de Lagrange em espaços lineares topológicos. 


\section{Capítulo 2}

\section{Preliminares}

Vejamos as notações e alguns resultados importantes sobre espaços de funções que utilizaremos durante nosso trabalho. O estudo sobre os espaços de funções foi baseado em [33] e para o caso de espaços de funções periódicas nos baseamos em [20].

Para $n \in Z_{+}^{*}$, consideremos

$$
\mathbb{R}_{+}^{n+1}=\left\{(x, z) \in \mathbb{R}^{n+1} \mid x \in \mathbb{R}^{n} \text { e } z>0\right\}
$$

Seja $U$ subconjunto aberto de $\mathbb{R}^{n}$. Denotaremos por $L^{2}(U)$ o espaço de Banach das funções $f: U \longrightarrow \mathbb{R}$, Lebesgue mensuráveis, satisfazendo

$$
\|f\|_{L^{2}(U)}=\left(\int_{U}|f|^{2} d x\right)^{\frac{1}{2}}<\infty .
$$

Consideremos também, o espaço de Hilbert

$$
X=\left\{u \in L^{2}(\Omega) \mid \int_{\Omega} u(x) d x=0\right\},
$$

onde o domínio $\Omega$ será o intervalo, o quadrado ou a bola, com as seguintes notações

$$
\begin{aligned}
\Omega_{i} & =(0, \pi) \\
\Omega_{q} & =(0, \pi) \times(0, \pi) \\
\Omega_{b} & =B(0, \pi) .
\end{aligned}
$$

O produto interno e a norma do espaço de funções $X$ são dados, respctivamente, por

$$
<u, v>=\int_{\Omega} u(x) v(x) d x \text { e }\|u\|=\sqrt{<u, u>}
$$

para qualquer $u, v \in X$.

Denotaremos por $K_{h}$, o cone de alura $h$, definido como

$$
K_{h}=\left\{\left(x^{\prime}, x_{n}\right) \in \mathbb{R}^{n} \quad\left|0<x_{n}<h, \quad\right| x^{\prime} \mid<a x_{n}\right\},
$$

$\operatorname{com} a>0, h>0$. 
Definição 1. ([33]) Um domínio $U \subset \mathbb{R}^{n}$ limitado é um domínio do tipo cone se existem domínios $U_{1}, \cdots, U_{M}$ e cones $C_{1}, \cdots, C_{M}$, que podem ser obtidos por rotações sobre o cone $K_{h}$, tais que

$$
\bigcup_{j=1}^{M} U_{j} \subset \partial U \quad e \quad\left(U_{j} \cap U\right)+C_{j} \subset U
$$

para qualquer $j=1, \cdots, M$.

Exemplo 1. $\Omega_{q}=(-\pi, \pi) \times(-\pi, \pi)$ é um domínio do tipo cone.

\subsection{Espaços de Sobolev, Slobodeckij e Hölder}

Seja $U \subset \mathbb{R}^{n}$ aberto. Durante nosso estudo usaremos frequentemente os espaços

$$
H^{s}(U)=H_{2}^{s}(U)
$$

com $s \in \mathbb{Z}_{+}$ou $s>0$ não inteiro. Por isso, nesta seção, definiremos estes espaços de funções e veremos algumas de suas propriedades.

Consideremos $S=S\left(\mathbb{R}^{n}\right)$ o conjunto das funções $f: \mathbb{R}^{n} \longrightarrow \mathbb{C}$ tais que $f \in C^{\infty}\left(\mathbb{R}^{n}\right)$ e satisfazem

$$
\sup _{x \in \mathbb{R}^{n}}\left|x^{\alpha} \partial^{\beta} f(x)\right|<\infty
$$

para quaisquer multi-índices $\alpha, \beta \in \mathbb{N}^{n}$. O conjunto $S$ é chamado Espaço de Schwartz. Denotaremos o dual de $S$ por $S^{\prime}=S^{\prime}\left(\mathbb{R}^{n}\right)$ que é o espaço das distribuições temperadas.

Definição 2. 1. Se $s \in \mathbb{Z}_{+}$, os espaços $H^{s}\left(\mathbb{R}^{n}\right)$ são os Espaços de Sobolev definidos como

$$
H^{s}\left(\mathbb{R}^{n}\right)=\left\{f \in S^{\prime}\left(\mathbb{R}^{n}\right) \mid\|f\|_{H^{s}\left(\mathbb{R}^{n}\right)}<\infty\right\},
$$

onde

$$
\|f\|_{H^{s}\left(\mathbb{R}^{n}\right)}=\left(\sum_{|\alpha| \leq s}\left\|D^{\alpha} f\right\|_{L^{2}\left(\mathbb{R}^{n}\right)}^{2}\right)^{\frac{1}{2}},
$$

para qualquer $f \in H^{s}\left(\mathbb{R}^{n}\right)$.

2. Se $s>0$ não inteiro, os espaços $H^{s}\left(\mathbb{R}^{n}\right)$ são os Espaços de Slobodeckij dados por

$$
H^{s}\left(\mathbb{R}^{n}\right)=\left\{f \in S^{\prime}\left(\mathbb{R}^{n}\right) \mid\|f\|_{H^{s}\left(\mathbb{R}^{n}\right)}<\infty\right\},
$$

onde

$$
\|f\|_{H^{s}\left(\mathbb{R}^{n}\right)}=\left\|\left(1+|x|^{2}\right)^{\frac{s}{2}} F f\right\|_{L^{2}\left(\mathbb{R}^{n}\right)},
$$

para qualquer $f \in H^{s}\left(\mathbb{R}^{n}\right)$, onde $F$ é a Transformada de Fourier em $\mathbb{R}^{n}$.

Tendo definido os espaços $H^{s}\left(\mathbb{R}^{n}\right)$ para $s>0$, podemos, então definir os espaços $H^{s}(U)$ como sendo a restrição dos espaços $H^{s}\left(\mathbb{R}^{n}\right)$.

Definição 3. Seja $U \subset \mathbb{R}^{n}$ aberto arbitrário. 
1. Se $s \in \mathbb{Z}_{+}$, os Espaços de Sobolev $H^{s}(U)$ são definidos como

$$
H^{s}(U)=\left\{f \in L^{2}(U) \mid \exists g \in H^{s}\left(\mathbb{R}^{n}\right) \quad \text { com } g(x)=f(x), \forall x \in U\right\},
$$

com a norma

$$
\|f\|_{H^{s}(U)}=\inf _{\substack{\left.g\right|_{U}=f \\ g \in H^{s}\left(\mathbb{R}^{n}\right)}}\|g\|_{H^{s}\left(\mathbb{R}^{n}\right)}
$$

para qualquer $f \in H^{s}(U)$.

2. Se $s>0$ não inteiro, os Espaços de Slobodeckij $H^{s}(U)$ são definidos como

$$
H^{s}(U)=\left\{f \in L^{2}(U) \mid \exists g \in H^{s}\left(\mathbb{R}^{n}\right) \quad \text { com } g(x)=f(x), \forall x \in U\right\},
$$

com a norma

$$
\|f\|_{H^{s}(U)}=\inf _{\substack{\left.g\right|_{U}=f \\ g \in H^{s}\left(\mathbb{R}^{n}\right)}}\|g\|_{H^{s}\left(\mathbb{R}^{n}\right)}
$$

para qualquer $f \in H^{s}(U)$.

Quando o domínio $U$ tem certas propriedades, os espaços $H^{s}(U)$ podem ser caracterizados com normas definidas em $U$. Para isso, consideraremos

$$
D(U)=\mathbb{C}_{0}^{\infty}(U)=\left\{f: U \longrightarrow \mathbb{R} \mid f \in C^{\infty}(U) \text { com suporte compacto }\right\},
$$

e $D^{\prime}(U)$ o dual de $D(U)$.

Lema 1. Seja $U \subset \mathbb{R}^{n}$ um domínio não limitado do tipo cone, limitado do tipo cone ou $C^{\infty}$-limitado.

1. Se $s \in \mathbb{Z}_{+}$, então

$$
H^{s}(U)=\left\{f \in D^{\prime}(U) \mid\|f\|_{H^{s}(U)}<\infty\right\}
$$

onde

$$
\|f\|_{H^{s}(U)}=\left(\|f\|_{L^{2}(U)}^{2}+\sum_{|\alpha|=s}\left\|D^{\alpha} f\right\|_{L^{2}(U)}^{2}\right)^{\frac{1}{2}},
$$

para qualquer $f \in H^{s}(U)$.

2. Se $s>0$ não inteiro, então

$$
H^{s}(U)=\left\{f \in D^{\prime}(U) \mid\|f\|_{H^{s}(U)}<\infty\right\}
$$

onde

$$
\|f\|_{H^{s}(U)}=\left(\|f\|_{L^{2}(U)}^{2}+\sum_{|\alpha|=[s]} \int_{U \times U} \frac{\left|D^{\alpha} f(x)-D^{\alpha} f(y)\right|^{2}}{|x-y|^{n+2}\{s\}} d x d y\right)^{\frac{1}{2}}
$$

para qualquer $f \in H^{s}(U)$, onde $s=[s]+\{s\}$ com $[s]$ inteiro e $\{s\} \in[0,1)$.

Vejamos agora a definição dos Espaços de Hölder. 
Definição 4. Seja $t \geq 0$, os Espaços de Hölder, denotados por $C^{t}\left(\mathbb{R}^{n}\right)$, são definidos da seguinte maneira

1. Se $t \in \mathbb{Z}_{+}$, então $C^{t}\left(\mathbb{R}^{n}\right)$ denota o completamento de $S\left(\mathbb{R}^{n}\right)$ na norma

$$
\|f\|_{C^{t}\left(\mathbb{R}^{n}\right)}=\sum_{|\alpha| \leq t} \sup _{x \in \mathbb{R}^{n}}\left|D^{\alpha} f(x)\right|
$$

2. Se $0<t=[t]+\{t\}$, então

$$
C^{t}\left(\mathbb{R}^{n}\right)=\left\{f \in C^{[t]}\left(\mathbb{R}^{n}\right) \mid\|f\|_{C^{t}\left(\mathbb{R}^{n}\right)}<\infty\right\}
$$

onde

$$
\|f\|_{C^{t}\left(\mathbb{R}^{n}\right)}=\|f\|_{C^{[t]}\left(\mathbb{R}^{n}\right)}+\sum_{|\alpha|=[t]} \sup _{x \neq y} \frac{\left|D^{\alpha} f(x)-D^{\alpha} f(y)\right|}{|x-y|^{\{t\}}} .
$$

Analogamente aos espaços de Sobolev e Slobodeckij, podemos definir os espaços de Hölder sobre $U$ através da restrição dos espaços $C^{t}\left(\mathbb{R}^{n}\right)$.

Definição 5. Seja $U \subset \mathbb{R}^{n}$ um domínio $C^{\infty}$-limitado. Então, o Espaço de Hölder sobre $U$, denotado por $\bar{C}^{t}(U)$, é dado por

$$
\bar{C}^{t}(U)=\left\{f \quad \mid \exists g \in C^{t}\left(\mathbb{R}^{n}\right) \quad \operatorname{com} g(x)=f(x), \forall x \in U\right\}
$$

com a norma

$$
\|f\|_{\bar{C}^{t}(U)}=\inf _{\substack{\left.g\right|_{U}=f \\ g \in C^{t}\left(\mathbb{R}^{n}\right)}}\|g\|_{C^{t}\left(\mathbb{R}^{n}\right)}
$$

\subsubsection{Imersões Contínuas e Compactas}

Durante nosso trabalho as imersões contínuas e compactas são de muita utilidade. Por isso, enunciaremos tais imersões para os espaços de Sobolev, Slobodeckij e Hölder.

As notações utilizadas para imersão contínua e compacta, são dadas, respectivamente, por $\hookrightarrow$ e $\stackrel{c}{\hookrightarrow}$.

Proposição 1. (Imersões Contínuas) Seja $U \subset \mathbb{R}^{n}$ um domínio arbitrário.

1. $S e-\infty<t \leq s<\infty$, então

$$
H^{s}(U) \hookrightarrow H^{t}(U)
$$

2. Se $t \geq 0, s>t+\frac{n}{2}$, então

$$
H^{s}(U) \hookrightarrow \bar{C}^{t}(U)
$$

3. Se $2 \leq q<\infty, s>0 e$

$$
s \geq n\left(\frac{1}{2}-\frac{1}{q}\right)
$$

então

$$
H^{s}(U) \hookrightarrow L^{q}(U)
$$


Proposição 2. (Imersões Compactas em domínios do tipo cone) Seja $U \subset \mathbb{R}^{n}$ um domínio limitado do tipo cone.

1. Se $0<s<\frac{1}{2}$ e $s<t<\infty$ então

$$
H^{t}(U) \stackrel{c}{\hookrightarrow} H^{s}(U)
$$

2. No caso em que $s=0$, temos

$$
H^{t}(U) \stackrel{c}{\hookrightarrow} L^{2}(U)
$$

3. Se $2 \leq q<\infty, s>0$ e

$$
s>n\left(\frac{1}{2}-\frac{1}{q}\right)
$$

então

$$
H^{s}(U) \stackrel{c}{\hookrightarrow} L^{q}(U)
$$

Proposição 3. (Imersões Compactas em domínios limitados suaves) Seja $U \subset \mathbb{R}^{n}$ um domínio $C^{\infty}$ limitado. Se $-\infty<s<t<\infty$, então

$$
H^{t}(U) \stackrel{c}{\hookrightarrow} H^{s}(U)
$$

Também, se $s>0$ e

$$
s>n\left(\frac{1}{2}-\frac{1}{q}\right)
$$

então

$$
H^{s}(U) \stackrel{c}{\hookrightarrow} L^{q}(U)
$$

\subsubsection{Teorema do Traço e Desigualdade de Poincaré}

Enunciaremos nesta subseção, o teorema do traço em $\mathbb{R}^{n}$ e em $U \subset \mathbb{R}^{n}$ domínio $C^{\infty}$-limitado. Estes teoremas podem ser encontrados em [33]. Nosso objetivo, é provar que faz sentido considerarmos o traço de uma função de $H^{s}(\Omega \times(0, \infty))$, onde $\Omega \subset \mathbb{R}^{n}$ é um aberto arbitrário e $s=1,2 . \cdots$. Também enunciaremos a Desigualdade de Poincaré para um domínio $U$ limitado em uma direção.

Teorema 2. (Teorema do Traço em $\mathbb{R}^{n}$ ) Sejam $s=1,2, \cdots$ e $x=\left(x_{1}, \cdots, x_{n-1}\right) \in \mathbb{R}^{n-1}$. Então, existe uma aplicação linear, contínua,

$$
\begin{aligned}
\gamma: H^{s}\left(\mathbb{R}^{n}\right) & \longrightarrow \prod_{j=0}^{s-1} H^{s-j-\frac{1}{2}}\left(\mathbb{R}^{n-1}\right) \\
f & \longmapsto \gamma(f)=\left\{f(x, 0), \frac{\partial f}{\partial x_{n}}(x, 0), \cdots, \frac{\partial^{s-1} f}{\partial x_{n}^{s-1}}(x, 0)\right\}
\end{aligned}
$$

Observação 1. O teorema anterior é válido para $s>0$ não necessariamente inteiro, desde que $s-j-\frac{1}{2}$ não seja inteiro. 
Teorema 3. (Teorema do Traço em U) Sejam $U \subset \mathbb{R}^{n}$ um domínio $C^{\infty}$-limitado e $\frac{1}{2}<s<\infty$. Então, existe uma aplicação linear, contínua,

$$
\begin{aligned}
\gamma: H^{s}(U) & \longrightarrow \prod_{j=0}^{\left[s-\frac{1}{2}\right]^{-}} H^{s-j-\frac{1}{2}}(\partial U) \\
f & \longmapsto \gamma(f)=\left\{\left.f\right|_{\partial U},\left.\frac{\partial f}{\partial \nu}\right|_{\partial U}, \cdots,\left.\frac{\partial^{\left[s-\frac{1}{2}\right]^{-}} f}{\partial \nu^{\left[s-\frac{1}{2}\right]^{-}}}\right|_{\partial U}\right\}
\end{aligned}
$$

onde $\nu$ é o vetor normal exterior em relação ao $\partial U$ e $\left[s-\frac{1}{2}\right]^{-}$é inteiro.

Com isso, temos o próximo lema sobre o traço das funções em $H^{s}(\Omega \times(0, \infty))$, para $s=1,2 . \cdots$.

Lema 2. Seja $u \in H^{s}(\Omega \times(0, \infty))$, onde $\Omega \subset \mathbb{R}^{n}$ aberto e $s=1,2 . \cdots$. Então,

$$
\gamma(u)=u(x, 0) \in H^{s-\frac{1}{2}}(\Omega \times\{0\})
$$

e existe $C>0$ tal que

$$
\|\gamma(u)\|_{H^{s-\frac{1}{2}(\Omega \times\{0\})}} \leq C\|u\|_{H^{s}(\Omega \times(0, \infty))} .
$$

Demonstração. Seja $u \in H^{s}(\Omega \times(0, \infty))$, então $u \in L^{2}(\Omega \times(0, \infty))$ e existe $g \in H^{s}\left(\mathbb{R}^{n+1}\right)$ tal que

$$
g(x, z)=u(x, z)
$$

para qualquer $(x, z) \in \Omega \times(0, \infty)$.

Pelo Teorema 2., $\gamma(g):=g(x, 0) \in H^{s-\frac{1}{2}}\left(\mathbb{R}^{n}\right)$ e existe $C>0$ tal que

$$
\|\gamma(g)\|_{H^{s-\frac{1}{2}\left(\mathbb{R}^{n}\right)}} \leq C\|g\|_{H^{s}\left(\mathbb{R}^{n+1}\right)}
$$

Temos, ainda que

$$
H^{s-\frac{1}{2}}\left(\mathbb{R}^{n}\right) \hookrightarrow L^{2}\left(\mathbb{R}^{n}\right),
$$

logo, $\gamma(g) \in L^{2}\left(\mathbb{R}^{n}\right)$, em particular $\left.\gamma(g)\right|_{\Omega \times\{0\}} \in L^{2}(\Omega \times\{0\})$.

Como $\Omega \times\{0\} \subset \mathbb{R}^{n}$, temos que

$$
\gamma(g)=g(x, 0)=u(x, 0):=\gamma(u)
$$

para qualquer $(x, 0) \in \Omega \times\{0\}$.

Então,

$$
\gamma(u):=\left.\gamma(g)\right|_{\Omega \times\{0\}} \in H^{s-\frac{1}{2}}(\Omega \times\{0\}) .
$$

Ainda,

$$
\begin{aligned}
& \|\gamma(u)\|_{H^{s-\frac{1}{2}}(\Omega \times\{0\})}=\inf _{\substack{\left.F \in H^{s-\frac{1}{2}}\left(\mathbb{R}^{n}\right) \\
F\right|_{\Omega \times\{0\}}=\gamma(u)}}\|F\|_{H^{s-\frac{1}{2}}\left(\mathbb{R}^{n}\right)} \\
& \leq\|\gamma(g)\|_{H^{s-\frac{1}{2}}\left(\mathbb{R}^{n}\right)} \\
& \leq C\|g\|_{H^{s}\left(\mathbb{R}^{n+1}\right)} \text {. }
\end{aligned}
$$


Observe que todas as contas que fizemos anteriormente para a função $g$, continuam valendo para uma função $h$ que tenha as mesmas propriedades da função $g$, ou seja, $h \in H^{s}\left(\mathbb{R}^{n+1}\right)$ tal que

$$
h(x, z)=u(x, z)
$$

para qualquer $(x, z) \in \Omega \times(0, \infty)$.

Logo, em (2.1), segue que

$$
\begin{aligned}
\|\gamma(u)\|_{H^{s-\frac{1}{2}}(\Omega \times\{0\})} & \leq C \inf _{\substack{\left.h \in H^{s}\left(\mathbb{R}^{n+1}\right) \\
h\right|_{\Omega \times(0, \infty)}=u}}\|h\|_{H^{s}\left(\mathbb{R}^{n+1}\right)} \\
& =C\|u\|_{H^{s}(\Omega \times(0, \infty))} .
\end{aligned}
$$

Portanto, existe $C>0$ tal que

$$
\|\gamma(u)\|_{H^{s-\frac{1}{2}}(\Omega \times\{0\})} \leq C\|u\|_{H^{s}(\Omega \times(0, \infty))},
$$

para qualquer $u \in H^{s}(\Omega \times(0, \infty))$.

A próxima proposição nos fornece a Desigualdade de Poincaré, a qual será muito útil na verificação de existência do mínimo de um funcional, que é uma das hipóteses do Teorema do Multiplicador de Lagrange.

Proposição 4. (Desigualdade de Poincaré) Seja $U \subset \mathbb{R}^{n}$ limitado em uma direção. Então, existe $C>0$ tal que

$$
\int_{U}|\tilde{v}(x)|^{2} d x \leq C\left(\int_{U} \tilde{v}(x) d x\right)^{2}+C \int_{U}|\nabla \tilde{v}(x)|^{2} d x,
$$

para qualquer $\tilde{v} \in H^{1}(U)$.

\subsection{Espaços de Sobolev das funções periódicas}

Denotaremos por $C_{p e r}^{\infty}\left(\mathbb{R}^{n}\right)$, o conjunto de todas as funções infinitamente diferenciáveis que são $2 \pi$-periódicas, isto é,

$$
\tilde{v}(x+2 \pi \cdot t)=\tilde{v}(x),
$$

para qualquer $x \in \mathbb{R}^{n}$ e $t=\left(t_{1}, \cdots, t_{n}\right) \in \mathbb{Z}^{n}$.

As funções do conjunto $C_{p e r}^{\infty}\left(\mathbb{R}^{n}\right)$, ficam unicamente determinadas no cubo

$$
Q^{n}=\left\{x \in \mathbb{R}^{n} \quad|\quad| x_{j} \mid \leq \pi, \forall j=1, \cdots, n\right\}
$$

Definição 6. Seja $s \in \mathbb{R}$. Definimos $H_{\text {per }}^{s}\left(\mathbb{R}^{n}\right)$ como sendo o fecho de $C_{\text {per }}^{\infty}\left(\mathbb{R}^{n}\right)$, com respeito a norma

$$
\|u\|_{H_{p e r}^{s}\left(\mathbb{R}^{n}\right)}=\left(\sum_{k \in \mathbb{Z}^{n}}\left(1+|k|^{2}\right)^{s}|\widehat{u}(k)|^{2}\right)^{\frac{1}{2}},
$$


onde $\widehat{u}(k)$ são os coeficientes de Fourier de u dados por

$$
\widehat{u}(k)=(2 \pi)^{-\frac{n}{2}} \int_{Q^{n}} e^{-i k \cdot x} u(x) d x,
$$

$e k \cdot x=k_{i} x_{1}+\cdots+k_{n} x_{n}$.

Em particular, $L_{\text {per }}^{2}\left(\mathbb{R}^{n}\right)=H_{\text {per }}^{0}\left(\mathbb{R}^{n}\right)$ é um espaço de Hilbert com o produto escalar

$$
<u, v>_{Q^{n}}=\int_{Q^{n}} u(x) \overline{v(x)} d x=\sum_{k \in \mathbb{Z}^{n}} \widehat{u}(k) \widehat{v}(k) .
$$

Estes espaços de Sobolev das funções periódicas, tem uma relação com os espaços de Sobolev e Slobodeckij em $Q^{n}$.

Para a demonstração do próximo lema, usaremos um resultado de [17], que nos diz que dados $a, b \in[0, \infty)$ e $s \geq 0$, existem constantes positivas $m_{s}$ e $M_{s}$ dependendo somente de $s$, tais que

$$
m_{s}\left(a^{s}+b^{s}\right) \leq(a+b)^{s} \leq M_{s}\left(a^{s}+b^{s}\right) .
$$

Lema 3. Seja $Q^{n} \subset \mathbb{R}^{n}$. Temos

1. Se $s \in \mathbb{Z}_{+}$, então $H_{\text {per }}^{s}\left(\mathbb{R}^{n}\right) \subset H^{s}\left(Q^{n}\right)$ e suas normas são equivalentes.

2. Se $0<s<1$, então $H_{\text {per }}^{s}\left(\mathbb{R}^{n}\right) \subset H^{s}\left(Q^{n}\right)$ e suas normas são equivalentes.

Demonstração. $\quad$ 1. Seja $u \in H_{p e r}^{s}\left(\mathbb{R}^{n}\right)$, temos que $u \in \overline{C_{p e r}^{\infty}\left(\mathbb{R}^{n}\right)}$. Por outro lado, temos que

$$
C^{\infty}\left(Q^{n}\right) \subset H^{s}\left(Q^{n}\right)
$$

então

$$
\overline{C^{\infty}\left(Q^{n}\right)} \subset H^{s}\left(Q^{n}\right),
$$

com a norma de $H^{s}\left(Q^{n}\right)$. Como $\overline{C_{p e r}^{\infty}\left(\mathbb{R}^{n}\right)} \subset \overline{C^{\infty}\left(Q^{n}\right)}$, segue que $u \in H^{s}\left(Q^{n}\right)$.

Temos ainda, usando (2.2), que

$$
\begin{aligned}
\sum_{k \in \mathbb{Z}^{n}}\left(1+|k|^{2}\right)^{s}|\widehat{u(k)}|^{2} & =\sum_{k \in \mathbb{Z}^{n}}\left(1+|i| k||^{2}\right)^{s}|\widehat{u(k)}|^{2} \\
& \leq M_{s} \sum_{k \in \mathbb{Z}^{n}}|\widehat{u(k)}|^{2}+M_{s} \sum_{k \in \mathbb{Z}^{n}}\left|(i|k|)^{s} \widehat{u(k)}\right|^{2} \\
& =M_{s} \sum_{k \in \mathbb{Z}^{n}}|\widehat{u(k)}|^{2}+M_{s} \sum_{k \in \mathbb{Z}^{n}}\left|\widehat{D^{s}(k)}\right|^{2} \\
& =M_{s}\left[\int_{Q^{n}}|u(x)|^{2} d x+\int_{Q^{n}}\left|D^{s} u(x)\right|^{2} d x\right] .
\end{aligned}
$$

Como $s \in \mathbb{Z}_{+}$, sabemos que

$$
\left|D^{s} u(x)\right|^{2}=\sum_{|\alpha|=s}\left|D^{\alpha} u(x)\right|^{2}
$$


Logo,

$$
\begin{aligned}
\sum_{k \in \mathbb{Z}^{n}}\left(1+|k|^{2}\right)^{s}|\widehat{u(k)}|^{2} & \leq M_{s}\left(\|u\|_{L^{2}\left(Q^{n}\right)}^{2}+\sum_{|\alpha|=s}\left\|D^{\alpha} u\right\|_{L^{2}\left(Q^{n}\right)}^{2}\right) \\
& =M_{s}\|u\|_{H^{s}\left(Q^{n}\right)}^{2}
\end{aligned}
$$

ou seja,

$$
\|u\|_{H_{p e r}^{s}\left(\mathbb{R}^{n}\right)} \leq \sqrt{M_{s}}\|u\|_{H^{s}\left(Q^{n}\right)} .
$$

Por outro lado, usando (2.2), também temos que

$$
\begin{aligned}
\sum_{k \in \mathbb{Z}^{n}}\left(1+|k|^{2}\right)^{s}|\widehat{u(k)}|^{2} & =\sum_{k \in \mathbb{Z}^{n}}\left(1+|i| k \|^{2}\right)^{s}|\widehat{u(k)}|^{2} \\
& \geq m_{s} \sum_{k \in \mathbb{Z}^{n}}|\widehat{u(k)}|^{2}+m_{s} \sum_{k \in \mathbb{Z}^{n}}\left|(i|k|)^{s} \widehat{u(k)}\right|^{2} \\
& =m_{s}\left(\|u\|_{L^{2}\left(Q^{n}\right)}^{2}+\sum_{|\alpha|=s}\left\|D^{\alpha} u\right\|_{L^{2}\left(Q^{n}\right)}^{2}\right)
\end{aligned}
$$

ou seja,

$$
\|u\|_{H_{p e r}^{s}\left(\mathbb{R}^{n}\right)} \geq \sqrt{m_{s}}\|u\|_{H^{s}\left(Q^{n}\right)}
$$

Portanto, $H_{\text {per }}^{s}\left(\mathbb{R}^{n}\right) \subset H^{s}\left(Q^{n}\right)$ e suas normas são equivalentes.

2. O fato de $H_{\text {per }}^{s}\left(\mathbb{R}^{n}\right) \subset H^{s}\left(Q^{n}\right)$, para $0<s<1$, segue pelo mesmo raciocínio usado no item 1 . Seja $u \in H_{\text {per }}^{s}\left(\mathbb{R}^{n}\right)$, usando propriedades de convolução e módulo, temos que

$$
\begin{aligned}
\int_{Q^{n}} \int_{Q^{n}} \frac{|u(x)-u(y)|^{2}}{|x-y|^{n+2 s}} d x d y & =\int_{Q^{n}} \int_{Q^{n}} \frac{|u(y-x)-u(y)|^{2}}{|x|^{n+2 s}} d x d y \\
& =\int_{Q^{n}} \frac{1}{|x|^{n+2 s}}\left[\int_{Q^{n}}|u(y-x)-u(y)|^{2} d y\right] d x
\end{aligned}
$$

Considere, para cada $x \in Q^{n}$, a função

$$
g_{x}(y)=u(y-x)-u(y)
$$

e observe que

$$
\begin{aligned}
\widehat{g_{x}}(k) & =(2 \pi)^{-\frac{n}{2}} \int_{Q^{n}} e^{-i k \cdot z} g_{x}(z) d z \\
& =(2 \pi)^{-\frac{n}{2}} \int_{Q^{n}} e^{-i k \cdot z} u(z-x) d z-(2 \pi)^{-\frac{n}{2}} \int_{Q^{n}} e^{-i k \cdot z} u(z) d z \\
& =(2 \pi)^{-\frac{n}{2}} \int_{Q^{n}} e^{-i k \cdot(z-x)} u(z) d z-\widehat{u}(k) \\
& =\left(e^{i k \cdot x}-1\right) \widehat{u}(k) .
\end{aligned}
$$


Assim, por (2.4) e pela Identidade de Parseval, segue em (2.3) que

$$
\begin{aligned}
\int_{Q^{n}} \int_{Q^{n}} \frac{|u(x)-u(y)|^{2}}{|x-y|^{n+2 s}} d x d y & =\int_{Q^{n}} \frac{1}{|x|^{n+2 s}}\left[\int_{Q^{n}} g_{x}(y) \overline{g_{x}(y)} d y\right] d x \\
& =\int_{Q^{n}} \frac{1}{|x|^{n+2 s}}\left[\sum_{k \in \mathbb{Z}^{n}}\left|\widehat{g_{x}}(k)\right|^{2}\right] d x \\
& =\sum_{k \in \mathbb{Z}^{n}}|\widehat{u}(k)|^{2}\left[\int_{Q^{n}} \frac{\left|e^{i k \cdot x}-1\right|^{2}}{|x|^{n+2 s}}\right] d x .
\end{aligned}
$$

Observe que se $|k|=0$, então

$$
\int_{Q^{n}} \int_{Q^{n}} \frac{|u(x)-u(y)|^{2}}{|x-y|^{n+2 s}} d x d y=0
$$

e assim

$$
\|u\|_{H^{s}\left(Q^{n}\right)}=\|u\|_{L^{2}\left(Q^{n}\right)}=\|u\|_{H_{p e r}^{s}\left(\mathbb{R}^{n}\right)} .
$$

Caso contrário, fazemos a mudança de variável $x=\frac{t}{|k|}$, logo

$$
\int_{Q^{n}} \frac{\left|e^{i k \cdot x}-1\right|^{2}}{|x|^{n+2 s}} d x=|k|^{2 s} \int_{Q^{n}} \frac{\left|e^{i k \cdot \frac{t}{|k|}}-1\right|^{2}}{|t|^{n+2 s}} d t=A_{s}|k|^{2 s} .
$$

Sendo assim,

$$
\int_{Q^{n}} \int_{Q^{n}} \frac{|u(x)-u(y)|^{2}}{|x-y|^{n+2 s}} d x d y=A_{s} \sum_{k \in \mathbb{Z}^{n}}|k|^{2 s}|\widehat{u}(k)|^{2} .
$$

Então, usando (2.2), segue que

$$
\begin{aligned}
\|u\|_{H_{p e r}^{s}\left(\mathbb{R}^{n}\right)}^{2} & \geq m_{s}\|u\|_{L_{p e r}^{2}\left(\mathbb{R}^{n}\right)}^{2}+m_{s} \sum_{k \in \mathbb{Z}^{n}}|k|^{2 s}|\widehat{u}(k)|^{2} \\
& =m_{s}\|u\|_{L^{2}\left(Q^{n}\right)}^{2}+\frac{m_{s}}{A_{s}} \int_{Q^{n}} \int_{Q^{n}} \frac{|u(x)-u(y)|^{2}}{|x-y|^{n+2 s}} d x d y \\
& \geq c_{s}\|u\|_{H^{s}\left(Q^{n}\right)}^{2} .
\end{aligned}
$$

Analogamente, existe $C_{s}>0$, tal que

$$
\|u\|_{H_{p e r}^{s}\left(\mathbb{R}^{n}\right)}^{2} \leq C_{s}\|u\|_{H^{s}\left(Q^{n}\right)}^{2}
$$

Portanto, $H_{\text {per }}^{s}\left(\mathbb{R}^{n}\right) \subset H^{s}\left(Q^{n}\right)$ e suas normas são equivalentes.

O lema anterior é importante, pois as imersões válidas para os espaços $H^{s}\left(Q^{n}\right)$, também são válidas para os espaços de Sobolev das funções periódicas $H_{\text {per }}^{s}\left(\mathbb{R}^{n}\right)$, para qualquer $s \in \mathbb{Z}_{+}$ou $0<s<1$. 
Consideremos também, o conjunto de todas as funções infinitamente diferenciáveis que são $2 \pi$ periódicas com respeito a variável $x$, isto é,

$$
\tilde{v}(x+2 \pi \cdot t, z)=\tilde{v}(x, z)
$$

para qualquer $(x, z) \in \mathbb{R}_{+}^{n+1}$ e $t=\left(t_{1}, \cdots, t_{n}\right) \in \mathbb{Z}^{n}$. Este conjunto será denotado por $C_{\text {per }}^{\infty}\left(\overline{\mathbb{R}_{+}^{n+1}}\right)$

Definição 7. Definimos $H_{\text {per }}^{s}\left(\mathbb{R}_{+}^{n+1}\right)$ como sendo o fecho de $C_{\text {per }}^{\infty}\left(\overline{\mathbb{R}_{+}^{n+1}}\right)$ com respeito a norma

$$
\|\tilde{u}\|_{H_{p e r}^{s}\left(\mathbb{R}_{+}^{n+1}\right)}=\left(\int_{0}^{\infty} \sum_{j=0}^{s}\left\|D_{z}^{j} \tilde{u}(\cdot, z)\right\|_{H_{\text {per }}^{s-j}\left(\mathbb{R}^{n}\right)}^{2} d z\right)^{\frac{1}{2}}
$$

para qualquer $\tilde{u} \in H_{\text {per }}^{s}\left(\mathbb{R}_{+}^{n+1}\right)$.

Em particular, $L_{\text {per }}^{2}\left(\mathbb{R}_{+}^{n+1}\right)=H_{\text {per }}^{0}\left(\mathbb{R}_{+}^{n+1}\right)$ é um espaço de Hilbert, com o produto interno dado por

$$
<\tilde{v}, \tilde{w}>_{Q^{n} \times(0, \infty)}=\int_{0}^{\infty} \int_{Q^{n}} \tilde{u}(x, z) \overline{\tilde{w}(x, z)} d x d z
$$

para todo $\tilde{u}, \tilde{w} \in L_{\text {per }}^{2}\left(\mathbb{R}_{+}^{n+1}\right)$.

Analogamente, aos espaços $H_{\text {per }}^{s}\left(\mathbb{R}^{n}\right)$, temos que $H_{\text {per }}^{s}\left(\mathbb{R}_{+}^{n+1}\right) \subset H^{s}\left(Q^{n} \times(0, \infty)\right)$, para qualquer $s \geq 0$.

Em particular, para $s=1$, temos que

$$
\begin{aligned}
\|u\|_{H_{p e r}^{1}\left(\mathbb{R}_{+}^{n+1}\right)}^{2} & =\int_{0}^{\infty} \sum_{j=0}^{1}\left\|D_{z}^{j} u(\cdot, z)\right\|_{H_{p e r}^{1-j}\left(\mathbb{R}^{n}\right)}^{2} d z \\
& =\int_{0}^{\infty} \int_{Q^{n}}|u(x, z)|^{2} d x d z+\int_{0}^{\infty} \int_{Q^{n}} \sum_{j=1}^{n}\left|\frac{\partial u}{\partial x_{j}}(x, z)\right|^{2} d x d z+\int_{0}^{\infty} \int_{Q^{n}}\left|u_{z}(x, z)\right|^{2} d x d z \\
& =\int_{0}^{\infty} \int_{Q^{n}}|u(x, z)|^{2} d x d z+\int_{0}^{\infty} \int_{Q^{n}}|\nabla u(x, z)|^{2} d x d z \\
& =\|u\|_{H^{1}\left(Q^{n} \times(0, \infty)\right)}^{2} .
\end{aligned}
$$

\subsubsection{Teorema do Traço para as funções periódicas}

Analogamente aos espaços de Sobolev e Slobodeckij, temos para os espaços de Sobolev das funções periódicas, o traço bem definido, como enunciaremos a seguir.

Lema 4. ([20])Considere o operador traço $\gamma_{\mu}$ sobre $C_{p e r}^{\infty}\left(\mathbb{R}_{+}^{n+1}\right)$ dado por

$$
\tilde{u} \longmapsto\left(\tilde{u}(x, 0),\left(D_{z} \tilde{u}\right)(x, 0), \cdots,\left(D_{z}^{\mu-1} \tilde{u}\right)(x, 0)\right),
$$

Então, se $s \geq \mu$, o operador $\gamma_{\mu}$ pode ser estendido continuamente a uma função limitada tal que

$$
\gamma_{\mu}: H_{p e r}^{s}\left(\mathbb{R}_{+}^{n+1}\right) \longrightarrow \prod_{j=1}^{\mu} H_{p e r}^{s-j+\frac{1}{2}}\left(\mathbb{R}^{n}\right) .
$$




\section{Capítulo 3}

\section{Cálculo Funcional}

Neste capítulo, estudaremos o operador Laplaciano com condição de fronteira de Neumann, denotado por $-\Delta_{N}$, nos domínios $\Omega_{i}, \Omega_{q}$ e $\Omega_{b}$.

Para facilitar a notação, quando o estudo independer do domínio, denotaremos o domínio somente por $\Omega$, quando necessário faremos a distinção.

Veremos algumas propriedades deste operador, com o objetivo de concluir que $-\Delta_{N}$ é um operador positivo e por isso faz sentido calcularmos sua raiz quadrada. Também veremos que o operador $\left(-\Delta_{N}\right)^{\frac{1}{2}}$ é dado como uma Série de Fourier, isto é,

$$
\begin{aligned}
\left(-\Delta_{N}\right)^{\frac{1}{2}}: D\left(\left(-\Delta_{N}\right)^{\frac{1}{2}}\right) \subset X & \longrightarrow X \\
u & \longmapsto\left(-\Delta_{N}\right)^{\frac{1}{2}} u=\sum_{i \in \mathcal{I}}^{\infty} \lambda_{i}^{\frac{1}{2}}<u, \varphi_{i}>\varphi_{i}
\end{aligned}
$$

onde $\mathcal{I}=\mathbb{N}^{*}$ em $\Omega_{i}, \mathcal{I}=(\mathbb{N} \times \mathbb{N}) \backslash\{(0,0)\}$ em $\Omega_{q}$ e $\mathcal{I}=\left(\mathbb{N}^{*} \times \mathbb{N}\right) \backslash\{(1,0)\}$ em $\Omega_{b},\left\{\varphi_{i}\right\}_{i \in \mathcal{I}}$ forma um sistema ortogonal completo de autofunções do operador $-\Delta_{N}$ em cada domínio $\Omega,\left\{\lambda_{i}\right\}_{i \in \mathcal{I}}$ são os correspondentes autovalores e

$$
D\left(\left(-\Delta_{N}\right)^{\frac{1}{2}}\right)=\left\{u \in X \mid \sum_{i \in \mathcal{I}}^{\infty} \lambda_{i}<u, \varphi_{i}>^{2}<\infty\right\} .
$$

Este capítulo será dividido em duas seções. Na primeira seção, estudaremos algumas propriedades do operador $-\Delta_{N}$ e concluiremos que este operador é um operador positivo. Na segunda seção, definiremos o operador $\left(-\Delta_{N}\right)^{\frac{1}{2}}$ como uma Série de Fourier, envolvendo as autofunções e os autovalores do operador $-\Delta_{N}$ nos domínios $\Omega$ e também daremos uma caracterização do $D\left(\left(-\Delta_{N}\right)^{\frac{1}{2}}\right)$.

\subsection{Estudo do Operador $-\Delta_{N}$}

Considere o operador

$$
\begin{array}{ccc}
-\Delta_{N}: D\left(-\Delta_{N}\right) \subset X & \longrightarrow & X \\
u & \longmapsto & -\Delta u
\end{array},
$$

onde, para $n$ sendo o vetor normal, unitário, exterior ao $\partial \Omega$, temos que

$$
D\left(-\Delta_{N}\right)=\left\{u \in H^{2}(\Omega) \quad\left|\frac{\partial u}{\partial n}\right|_{\partial \Omega}=0 \text { e } \int_{\Omega} u(x) d x=0\right\}
$$


Durante o texto, usaremos frequentemente a notação $u_{\Omega}$, tal que

$$
u_{\Omega}=\int_{\Omega} u(x) d x
$$

para qualquer domínio $\Omega$.

Lema 5. O operador $-\Delta_{N}$ é monótono, simétrico e satisfaz $R\left(I-\Delta_{N}\right)=X$.

Demonstração. Primeiramente, observemos que o operador $-\Delta_{N}$ é densamente definido.

Seja $u \in X \in L^{2}(\Omega)$, logo como $\overline{C_{0}^{\infty}(\Omega)}=L^{2}(\Omega)$, segue que existe $\left\{u_{n}\right\} \in C_{0}^{\infty}(\Omega)$ tal que

$$
\left\|u_{n}-u\right\|_{L^{2}(\Omega)} \stackrel{n \rightarrow \infty}{\longrightarrow 0}
$$

onde $C_{0}^{\infty}(\Omega)$ é o conjunto das funções infinitamente diferenciáveis com suporte compacto em $\Omega$.

Considere a função

$$
v_{n}(x)=u_{n}(x)-\frac{\left(u_{n}\right)_{\Omega}}{m(\Omega)}
$$

e observe que

1. $v_{n} \in C^{\infty}(\Omega) \subset H^{2}(\Omega)$, para qualquer $n \in \mathbb{N}$.

2. $\left(v_{n}\right)_{\Omega}=0$, para qualquer $n \in \mathbb{N}$.

3.

$$
\left.\frac{\partial v_{n}}{\partial n}\right|_{\partial \Omega}=\left.\frac{\partial u_{n}}{\partial n}\right|_{\partial \Omega}=0,
$$

pois $u_{n}$ tem suporte compacto em $\Omega$, para qualquer $n \in \mathbb{N}$.

Então, de 1., 2. e 3. segue que $\left\{v_{n}\right\} \subset D\left(-\Delta_{N}\right)$ e ainda

$$
\begin{aligned}
\left\|u-v_{n}\right\| & \leq\left\|u-u_{n}\right\|_{L^{2}(\Omega)}+\left\|\frac{u_{\Omega}}{m(\Omega)}-\frac{\left(u_{n}\right)_{\Omega}}{m(\Omega)}\right\|_{L^{2}(\Omega)} \\
& \leq 2\left\|u-u_{n}\right\|_{L^{2}(\Omega)} \stackrel{n \rightarrow \infty}{\longrightarrow 0,}
\end{aligned}
$$

logo, temos que $X=\overline{D\left(-\Delta_{N}\right)}$.

Por outro lado, sejam $u, v \in D\left(-\Delta_{N}\right)$. Temos usando Integração por Partes para $\Omega_{i}$ e $\Omega_{q}$, e as Fórmulas de Green para $\Omega_{b}$, que

$$
\begin{aligned}
<-\Delta_{N} u, u> & =\int_{\Omega}|\nabla u(x)|^{2} d x \geq 0 \\
<-\Delta_{N} u, v> & =\int_{\Omega} \nabla u(x) \nabla v(x) d x \\
& =<u,-\Delta_{N} v>
\end{aligned}
$$

logo, o operador $-\Delta_{N}$ é um operador monótono e simétrico, respectivamente. 
Seja $g \in X$, queremos encontrar $u \in D\left(-\Delta_{N}\right)$ tal que $\left(I-\Delta_{N}\right) u=g$, isto é, queremos encontrar $u \in H^{2}(\Omega)$ tal que

$$
\left\{\begin{array}{l}
-\Delta u+u=g \quad \text { em } \quad \Omega \\
\left.\frac{\partial u}{\partial n}\right|_{\partial \Omega}=0 \\
u_{\Omega}=0
\end{array} .\right.
$$

Considere $a: \Xi \times \Xi \longrightarrow \mathbb{R}$ tal que

$$
a(u, v)=\int_{\Omega} \nabla u(x) \nabla v(x) d x+\int_{\Omega} u(x) v(x) d x
$$

onde $\Xi=\left\{u \in H^{1}(\Omega) \mid u_{\Omega}=0\right\}$ é um Espaço de Hilbert.

A função $a$ é bilinear e usando a Desigualdade de Hölder, para qualquer $u, v \in \Xi$ vale que

1.

$$
\begin{aligned}
|a(u, v)| & \leq \int_{\Omega}|\nabla u(x) \nabla v(x)| d x+\int_{\Omega}|u(x) v(x)| d x \\
& \leq 2\|u\|_{H^{1}(\Omega)}\|v\|_{H^{1}(\Omega)}
\end{aligned}
$$

2.

$$
\begin{aligned}
|a(u, u)| & =\int_{\Omega}|\nabla u(x)|^{2} d x+\int_{\Omega}|u(x)|^{2} d x \\
& =\|u\|_{H^{1}(\Omega)}^{2} .
\end{aligned}
$$

Então, tomando $F: \Xi \longrightarrow \mathbb{R}$ tal que

$$
F(v)=\int_{\Omega} g(x) v(x) d x
$$

para qualquer $v \in \Xi$, temos que $F \in \Xi^{\prime}$ e pelo Teorema de Lax-Milgran, segue que existe uma única $u \in \Xi$ tal que

$$
\int_{\Omega} \nabla u(x) \nabla v(x) d x+\int_{\Omega} u(x) v(x) d x=\int_{\Omega} g(x) v(x) d x
$$

para qualquer $v \in \Xi$ e em particular para qualquer $v \in H^{1}(\Omega)$.

Então, por [6], temos que $u \in H^{2}(\Omega)$ e ainda usando o Método de Integração por Partes para $\Omega_{i}$ e $\Omega_{q}$, e as Fórmulas de Green para $\Omega_{b}$, em (3.2) segue que

$$
\int_{\Omega}[\Delta u(x)-u(x)+g(x)] v(x) d x=\int_{\partial \Omega} \frac{\partial u}{\partial n}(x) v(x) d \sigma,
$$

para qualquer $v \in H^{1}(\Omega)$. 
Em particular, para $v \in C_{0}^{\infty}(\Omega) \subset H^{1}(\Omega)$, segue em (3.3) que

$$
\int_{\Omega}[\Delta u(x)-u(x)+g(x)] v(x) d x=0 \Longrightarrow-\Delta u+u=g \text { quase sempre. }
$$

Voltando à (3.3), temos que

$$
\int_{\partial \Omega} \frac{\partial u}{\partial n}(x) v(x) d x=0
$$

para qualquer $v \in H^{1}(\Omega)$. Em particular, para

$$
v=\frac{\partial u}{\partial n} \in H^{1}(\Omega),
$$

segue que

$$
\int_{\partial \Omega}\left|\frac{\partial u}{\partial n}(x)\right|^{2} d x=\left.0 \Longrightarrow \frac{\partial u}{\partial n}\right|_{\partial \Omega}=0 \text { quase sempre. }
$$

Portanto, $u$ satisfaz

$$
\left\{\begin{array}{l}
-\Delta u+u=g \quad \text { em } \Omega \\
\left.\frac{\partial u}{\partial n}\right|_{\partial \Omega}=0 \\
u_{\Omega}=0
\end{array},\right.
$$

ou seja, $R\left(I-\Delta_{N}\right)=X$.

Pelo lema anterior, temos a seguinte proposição.

Proposição 5. O operador $-\Delta_{N}$ é um operador auto-adjunto.

Demonstração. A demonstração segue do Lema 5. e de [26], página 20.

Proposição 6. O operador $-\Delta_{N}$ é limitado inferiormente, isto é, existe $m \in \mathbb{R}$ tal que para qualquer $u \in D\left(-\Delta_{N}\right)$, vale

$$
<-\Delta_{N} u, u>\geq m\|u\|^{2}
$$

Demonstração. Seja $u \in D\left(-\Delta_{N}\right)$, usando o Método de Integração por Partes para $\Omega_{i}$ e $\Omega_{q}$, e as Fórmulas de Green para $\Omega_{b}$, temos que

$$
\begin{aligned}
<-\Delta_{N} u, u> & =-\int_{\Omega} \Delta u(x) u(x) d x \\
& =\int_{\Omega}|\nabla u(x)|^{2} d x .
\end{aligned}
$$

Para concluirmos a demonstração, usaremos a Desigualdade de Poincaré Generalizada em cada caso de $\Omega$.

1. Se $\Omega=\Omega_{i}$, considerando $\tilde{u}$ como sendo a extensão par, $2 \pi$-periódica de $u$, temos que $\tilde{u}_{(0,2 \pi)}=0$ e $\tilde{u} \in H^{1}(0,2 \pi)$, então pela Desigualdade de Wirtinger, segue que

$$
\int_{0}^{2 \pi}|\tilde{u}(x)|^{2} d x \leq \int_{0}^{2 \pi}\left|\tilde{u}_{x}(x)\right|^{2} d x,
$$


para qualquer $\tilde{u} \in H^{1}(0,2 \pi)$, logo, tomando $m=1$ e usando a periodicidade de $\tilde{u}$, temos que

$$
m \int_{\Omega_{i}}|u(x)|^{2} \leq \int_{\Omega_{i}}|\nabla u(x)|^{2} d x .
$$

2. Se $\Omega=\Omega_{b}$, temos por [11], que existe $M>0$, tal que

$$
\left\|u-\frac{1}{m\left(\Omega_{b}\right)} u_{\Omega_{b}}\right\|_{L^{2}\left(\Omega_{b}\right)} \leq M\|\nabla u\|_{L^{2}\left(\Omega_{b}\right)} \Longrightarrow\|u\|_{L^{2}\left(\Omega_{b}\right)} \leq \frac{1}{m\left(\Omega_{b}\right)}\left\|u_{\Omega_{b}}\right\|_{L^{2}\left(\Omega_{b}\right)}+M\|\nabla u\|_{L^{2}\left(\Omega_{b}\right)}
$$

Como $u_{\Omega_{b}}=0$, tomando $m=\min \left\{1, \frac{1}{M^{2}}\right\}$, segue que

$$
m \int_{\Omega_{b}}|u(x, y)|^{2} d x d y \leq \int_{\Omega_{b}}|\nabla u(x, y)|^{2} d x d y
$$

3. Se $\Omega=\Omega_{q}$, temos por [22], que existe $N>0$, tal que

$$
\|u\|_{H^{1}\left(\Omega_{q}\right)}^{2} \leq N^{2}\left(\|\nabla u\|_{L^{2}\left(\Omega_{q}\right)}^{2}+\left|\int_{\Omega_{q}} u(x, y) d x d y\right|^{2}\right) \Longrightarrow\|u\|_{H^{1}\left(\Omega_{q}\right)}^{2} \leq N^{2}\|\nabla u\|_{L^{2}\left(\Omega_{q}\right)}^{2} .
$$

Como, $\|u\|_{L^{2}\left(\Omega_{q}\right)}^{2} \leq\|u\|_{H^{1}\left(\Omega_{q}\right)}^{2}$, segue para $m=\min \left\{1, \frac{1}{N^{2}}\right\}$, que

$$
m \int_{\Omega_{q}}|u(x, y)|^{2} d x d y \leq \int_{\Omega_{q}}|\nabla u(x, y)|^{2} d x d y
$$

Portanto, em cada caso de $\Omega$, existe $m>0$ tal que em (3.4) vale

$$
<-\Delta_{N} u, u>\geq m\|u\|_{L^{2}(\Omega)}^{2}
$$

Com isso, temos o seguinte teorema.

Teorema 4. O operador $-\Delta_{N}$ é um operador positivo.

Demonstração. Pelas Proposições 5., 6., e o Teorema 14., temos que $-\Delta_{N}$ é um operador setorial tal que

$$
\operatorname{Re}\left(\sigma\left(-\Delta_{N}\right)\right) \geq m
$$

Temos também, por [9], que $\sigma\left(-\Delta_{N}\right) \subset[m,+\infty)$, logo,

$$
\sigma\left(\Delta_{N}\right) \subset(-\infty,-m] \Longrightarrow[0,+\infty) \subset \rho\left(\Delta_{N}\right)
$$

Por definição de Operador Setorial, temos que o operador $-\Delta_{N}$ é fechado, densamente definido e para algum $0<\phi<\frac{\pi}{2}, M \geq 1$, vale que

$$
S_{m, \phi}=\{\lambda \in \mathbb{C}|\phi \leq| \arg (\lambda-m) \mid \leq \pi, \lambda \neq m\} \subset \rho\left(-\Delta_{N}\right)
$$


e

$$
\left\|\left(\lambda I+\Delta_{N}\right)^{-1}\right\| \leq \frac{M}{|\lambda-m|}
$$

para qualquer $\lambda \in S_{m, \phi}$.

Assim, dado $s \in \mathbb{R}_{+}$temos, pelo fato de $m>0$, que $\lambda=-s \in \mathbb{R}_{-} \subset S_{m, \phi}$, logo,

$$
\left\|\left(s I-\Delta_{N}\right)^{-1}\right\| \leq \frac{M}{|s+m|}
$$

Então,

$$
\begin{aligned}
(1+s)\left\|\left(s I-\Delta_{N}\right)^{-1}\right\| & \leq M\left[\frac{1}{|s+m|}+\frac{s}{|s+m|}\right] \\
& \leq M\left[\frac{1}{m}+1\right]
\end{aligned}
$$

para qualquer $s \in \mathbb{R}_{+}$, ou seja, existe $\widetilde{M} \geq 1$, onde

$$
\widetilde{M}=\frac{(1+m)}{m} M
$$

tal que

$$
(1+s)\left\|\left(s I-\Delta_{N}\right)^{-1}\right\| \leq \widetilde{M}
$$

para qualquer $s \in \mathbb{R}_{+}$.

Portanto, $-\Delta_{N}$ é um operador positivo.

\subsection{Raiz Quadrada do operador $-\Delta_{N}$}

Como vimos na seção anterior, o operador $-\Delta_{N}$ é um operador positivo, logo, concluímos que faz sentido considerarmos a raiz quadrada de $-\Delta_{N}$, ou seja, o operador

$$
\left(-\Delta_{N}\right)^{\frac{1}{2}}: D\left(\left(-\Delta_{N}\right)^{\frac{1}{2}}\right) \subset X \longrightarrow X
$$

está bem definido.

Para escrevermos $\left(-\Delta_{N}\right)^{\frac{1}{2}}$ como uma Série de Fourier e caracterizarmos o $D\left(\left(-\Delta_{N}\right)^{\frac{1}{2}}\right)$, usaremos a teoria de Cálculo Funcional de [15] e [27].

Sabemos que o operador $-\Delta_{N}$ é setorial e $\operatorname{Re}\left(\sigma\left(-\Delta_{N}\right)\right)>0$, então por [15] temos que

$$
\left(-\Delta_{N}\right)^{-\frac{1}{2}}: X \longrightarrow X
$$

é um operador linear, limitado tal que

$$
\left(-\Delta_{N}\right)^{-\frac{1}{2}}=\frac{1}{\pi} \int_{0}^{\infty} \lambda^{-\frac{1}{2}}\left(\lambda I-\Delta_{N}\right)^{-1} d \lambda
$$

Como nosso objetivo é usar a teoria espectral, vamos inicialmente, exibir um sistema ortogonal completo para $X$, formado pelas autofunções do operador $-\Delta_{N}$ e os respectivos autovalores, em cada caso do domínio $\Omega$. 
Proposição 7. Considere o operador $-\Delta_{N}$ em $\Omega$.

1. Se $\Omega=\Omega_{i}$, então suas autofunções ortonormais $\varphi_{j}$ e seus respectivos autovalores $\lambda_{j}$ são dados por

$$
\varphi_{j}(x)=\sqrt{\frac{2}{\pi}} \cos (j x) \quad e \quad \lambda_{j}=j^{2},
$$

para qualquer $j \geq 1$.

2. Se $\Omega=\Omega_{q}$, então suas autofunções ortonormais $\varphi_{j k}$ e seus respectivos autovalores $\lambda_{j k}$ são dados por

$$
\varphi_{j k}(x, y)=\beta_{j k} \cos (j x) \cos (k y) \quad e \quad \lambda_{j k}=j^{2}+k^{2},
$$

onde

$$
\beta_{j k}=\left\{\begin{array}{l}
\frac{\sqrt{2}}{\pi} \quad \text { se } \quad k=0 \text { ou } j=0 \\
\frac{2}{\pi} \quad \text { se } \quad k, j \geq 1
\end{array},\right.
$$

para qualquer $(j, k) \in(\mathbb{N} \times \mathbb{N}) \backslash\{(0,0)\}$.

3. Se $\Omega=\Omega_{b}$, então suas autofunções ortonormais $\varphi_{j k}$ são dadas por

$$
\varphi_{j 0}(x, y)=w_{j 0}(r, \theta)=\bar{A}_{j 0} J_{0}\left(\frac{\mu_{j 0} r}{\pi}\right)
$$

onde

$$
\bar{A}_{j 0}=\frac{1}{\pi^{\frac{3}{2}}\left|J_{0}\left(\mu_{j 0}\right)\right|},
$$

para qualquer $j \geq 2$,

$$
\varphi_{j k}(x, y)=w_{j k}(r, \theta)=\left\{\begin{array}{l}
\bar{A}_{j k} J_{k}\left(\frac{\mu_{j k} r}{\pi}\right) \cos (k \theta) \\
\bar{B}_{j k} J_{k}\left(\frac{\mu_{j k} r}{\pi}\right) \sin (k \theta)
\end{array},\right.
$$

para qualquer $j, k \geq 1$, onde

$$
\bar{A}_{j k}=\bar{B}_{j k}=\frac{\sqrt{2} \mu_{j k}}{\pi^{\frac{3}{2}}\left(\mu_{j k}^{2}-k^{2}\right)^{\frac{1}{2}}\left|J_{k}\left(\mu_{j k}\right)\right|}
$$

e seus respectivos autovalores $\lambda_{j k}$ são dados por

$$
\lambda_{j k}=\left(\frac{\mu_{j k}}{\pi}\right)^{2}
$$

para qualquer $(j, k) \in\left(\mathbb{N}^{*} \times \mathbb{N}\right) \backslash\{(1,0)\}$, onde $\mu_{j k}$ são os zeros estritamente positivos de $J_{k}^{\prime}$. $E$ ainda, estas autofunções formam um sistema ortogonal completo para $X$. 
Demonstração. O caso em que o domínio é $\Omega_{i}$ é facilmente verificado, pois sabemos que as funções senos, cossenos e constantes formam um sistema ortogonal completo para $L^{2}(-\pi, \pi)$. Sendo assim, como qualquer função de $X$ pode ser estendida par em $(-\pi, \pi)$, e usando o fato desta função ter média nula, verificamos que os coeficientes de Fourier desta função, que envolvem as funções senos e constantes se anulam, logo $\left\{\varphi_{j}\right\}_{j \geq 1}$ forma um sistema ortogonal completo para $X$ no caso $\Omega_{i}$.

Por outro lado, temos em [14] que as autofunções e os respectivos autovalores do operador $-\Delta_{N}$ são dados desta forma em $\Omega_{q}$ e $\Omega_{b}$, considerando que estamos estudando as autofunções com média nula.

Vamos, então verificar que estas autofunções formam um sistema ortogonal completo para $X$, ou seja, mostrar que elas formam um conjunto completo em $X$, já que a ortonormalidade é fácil de se verificar. A demonstração será feita para cada caso de $\Omega$.

1. $\Omega=\Omega_{q}$.

Temos por [32], que vale a Identidade de Parseval em $L^{2}\left(Q^{2}\right)$, isto é,

$$
\frac{1}{\pi^{2}}\|f\|_{L^{2}\left(Q^{2}\right)}^{2}=\sum_{j, k \geq 0}^{\infty} \delta_{j k}\left(a_{j k}^{2}+b_{j k}^{2}+c_{j k}^{2}+d_{j k}^{2}\right),
$$

para qualquer $f \in L^{2}\left(Q^{2}\right)$, onde

$$
\begin{aligned}
a_{j k} & =\frac{1}{\pi^{2}} \int_{Q^{2}} f(x, y) \cos (j x) \cos (k y) d x d y, \\
b_{j k} & =\frac{1}{\pi^{2}} \int_{Q^{2}} f(x, y) \sin (j x) \cos (k y) d x d y, \\
c_{j k} & =\frac{1}{\pi^{2}} \int_{Q^{2}} f(x, y) \cos (j x) \sin (k y) d x d y, \\
d_{j k} & =\frac{1}{\pi^{2}} \int_{Q^{2}} f(x, y) \sin (j x) \sin (k y) d x d y
\end{aligned}
$$

para todo $j, k \geq 0 \mathrm{e}$

$$
\delta_{j k}=\left\{\begin{array}{l}
\frac{1}{4} \quad \text { se } \quad j=k=0 \\
\frac{1}{2} \quad \text { se } j>0, k=0 \text { ou } j=0, k>0 \\
1 \quad \text { se } \quad j>0, k>0
\end{array} .\right.
$$

Seja $g \in X$, tome sua extensão par nas variáveis $x$ e $y$, isto é,

$$
f(x, y)=\left\{\begin{array}{llllll}
g(x, y) & \text { se } & (x, y) \in & {[0, \pi]} & \times & {[0, \pi]} \\
g(-x, y) & \text { se } & (x, y) \in & {[-\pi, 0]} & \times & {[0, \pi]} \\
g(x,-y) & \text { se } & (x, y) \in & {[0, \pi]} & \times & {[-\pi, 0]} \\
g(-x,-y) & \text { se } & (x, y) \in & {[-\pi, 0]} & \times & {[-\pi, 0]}
\end{array} .\right.
$$


Como $f$ é par em $x$ e $y$, temos que $b_{j k}=c_{j k}=d_{j k}=0$, para todo $j, k \geq 0$, e pelo fato de $g$ ter média nula, temos também que $a_{00}=0$. Então,

$$
\frac{4}{\pi^{2}}\|g\|_{L^{2}\left(\Omega_{q}\right)}^{2}=\sum_{(j, k) \in \mathcal{I}}^{\infty} \delta_{j k} a_{j k}^{2},
$$

onde

$$
a_{j k}=\frac{4}{\pi^{2}} \int_{\Omega_{q}} g(x, y) \cos (j x) \cos (k y) d x d y \text {. }
$$

Portanto,

$$
\|g\|_{L^{2}\left(\Omega_{q}\right)}^{2}=\sum_{(j, k) \in \mathcal{I}}^{\infty}\left|<g, \varphi_{j k}>\right|^{2},
$$

isto é, vale a Identidade de Parseval em $X$, logo, $\left\{\varphi_{j k}\right\}_{(j, k) \in \mathcal{I}}$ forma um sistema ortogonal completo para $X$ em $\Omega_{q}$.

2. $\Omega=\Omega_{b}$.

Seja $u \in X$ tal que $<u, \varphi_{j k}>=0$, para qualquer $(j, k) \in \mathcal{I}$. Logo,

$$
\int_{0}^{2 \pi} \int_{0}^{\pi} r U(r, \theta) w_{j k}(r, \theta) d r d \theta=0
$$

para qualquer $(j, k) \in \mathcal{I}$.

Sabemos, para $p \in \mathbb{R}$, que

$$
\left\{J_{p}\left(\frac{\mu_{j p}}{\pi} \cdot\right)\right\}_{j=1}^{\infty}
$$

forma um sistema ortogonal completo para $L^{2}((0, \pi) ; r)$, onde $L^{2}((0, \pi) ; r)$ é o espaço $L^{2}((0, \pi))$ com peso $r$.

Assim,

(a) se $k=0$ e $j \geq 2$, então

$$
\int_{0}^{\pi} r h(r) J_{0}\left(\frac{\mu_{j 0}}{\pi} r\right) d r=0
$$

para qualquer $j \geq 2$, onde

$$
h(r)=\int_{0}^{2 \pi} U(r, \theta) d \theta .
$$

Como $\mu_{10}=0$, temos que $J_{0}\left(\frac{\mu_{10}}{\pi} r\right)=1 \mathrm{e}$

$$
\int_{0}^{\pi} r h(r) J_{0}\left(\frac{\mu_{10}}{\pi} r\right) d r=\int_{0}^{2 \pi} \int_{0}^{\pi} r U(r, \theta) d r d \theta=0
$$

pois $u_{\Omega_{b}}=0$.

Então,

$$
\left\langle h, J_{0}\left(\frac{\mu_{j 0}}{\pi} \cdot\right)\right\rangle_{L^{2}((0, \pi) ; r)}=0
$$


para qualquer $j \geq 1, \operatorname{logo}$

$$
\int_{0}^{2 \pi} U(r, \theta) d \theta=0
$$

para qualquer $r \in(0, \pi)$.

(b) se

$$
w_{j k}=J_{k}\left(\frac{\mu_{j k}}{\pi} r\right) \cos (k \theta),
$$

então usando a mesma idéia do caso anterior, segue que

$$
\int_{0}^{2 \pi} U(r, \theta) \cos (k \theta) d \theta=0
$$

para qualquer $r \in(0, \pi), k \geq 1$.

(c) se

$$
w_{j k}=J_{k}\left(\frac{\mu_{j k}}{\pi} r\right) \sin (k \theta),
$$

temos que

$$
\int_{0}^{2 \pi} U(r, \theta) \sin (k \theta) d \theta=0
$$

para qualquer $r \in(0, \pi), k \geq 1$.

Usando o fato de que

$$
\left\{\frac{1}{\sqrt{2 \pi}}, \frac{\cos \theta}{\sqrt{\pi}}, \frac{\sin \theta}{\sqrt{\pi}}, \frac{\cos (2 \theta)}{\sqrt{\pi}}, \frac{\sin (2 \theta)}{\sqrt{\pi}}, \cdots\right\}
$$

forma um sistema ortogonal completo para $L^{2}([-\pi, \pi])$, temos que $U(r, \theta)=0$ para qualquer $(r, \theta) \in(0, \pi) \times(0,2 \pi)$. Portanto, as autofunções do operador $-\Delta_{N}$ formam um sistema ortogonal completo para $X$ em $\Omega_{b}$.

A próxima proposição descreverá o operador $\left(-\Delta_{N}\right)^{-\frac{1}{2}}$, como uma Série de Fourier formada pelas autofunções e respectivos autovalores do operador $-\Delta_{N}$.

Proposição 8. Para qualquer $u \in X$, temos que

$$
\left(-\Delta_{N}\right)^{-\frac{1}{2}} u=\sum_{i \in \mathcal{I}}^{\infty} \lambda_{i}^{-\frac{1}{2}}<u, \varphi_{i}>\varphi_{i}
$$

onde $\left\{\varphi_{i}\right\}_{i \in \mathcal{I}}$ é um sistema ortogonal completo de $X$, formado por autofunções do operador $-\Delta_{N} e$ $\left\{\lambda_{i}\right\}_{i \in \mathcal{I}}$ são os respectivos autovalores.

Demonstração. Seja $\varphi_{i}$ autofunção do operador $-\Delta_{N}$, para qualquer $i \in \mathcal{I}$. Como $\varphi_{i} \in X$, temos que

$$
\left(-\Delta_{N}\right)^{-\frac{1}{2}} \varphi_{i}=\frac{1}{\pi} \int_{0}^{\infty} \lambda^{-\frac{1}{2}}\left(\lambda I-\Delta_{N}\right)^{-1} \varphi_{i} d \lambda
$$

Mas,

$$
-\Delta_{N} \varphi_{i}=\lambda_{i} \varphi_{i}
$$


para qualquer $i \in \mathcal{I}$ e como $\lambda \in[0, \infty) \subset \rho\left(\Delta_{N}\right)$, temos que

$$
\left(\lambda I-\Delta_{N}\right)^{-1} \varphi_{i}=\frac{1}{\lambda+\lambda_{i}} \varphi_{i}
$$

para qualquer $i \in \mathcal{I}$.

Então,

$$
\left(-\Delta_{N}\right)^{-\frac{1}{2}} \varphi_{i}=\frac{\varphi_{i}}{\pi} \int_{0}^{\infty} \frac{\lambda^{-\frac{1}{2}}}{\lambda+\lambda_{i}} d \lambda=\lambda_{i}^{-\frac{1}{2}} \varphi_{i}
$$

para qualquer $i \in \mathcal{I}$.

Seja $u \in X, \log o$

$$
u=\sum_{i \in \mathcal{I}}^{\infty}<u, \varphi_{i}>\varphi_{i}
$$

Assim, considerando a soma parcial

$$
s_{m}=\sum_{i \in \mathcal{I}}^{m}<u, \varphi_{i}>\varphi_{i}
$$

temos que

$$
\left\|\left(-\Delta_{N}\right)^{-\frac{1}{2}} u-\sum_{i \in \mathcal{I}}^{m} \lambda_{i}^{-\frac{1}{2}}<u, \varphi_{i}>\varphi_{i}\right\|=\left\|\left(-\Delta_{N}\right)^{-\frac{1}{2}} u-\left(-\Delta_{N}\right)^{-\frac{1}{2}} s_{m}\right\| \leq K\left\|u-s_{m}\right\| \stackrel{m \rightarrow \infty}{\longrightarrow} 0 .
$$

Portanto,

$$
\left(-\Delta_{N}\right)^{-\frac{1}{2}} u=\sum_{i \in \mathcal{I}}^{\infty} \lambda_{i}^{-\frac{1}{2}}<u, \varphi_{i}>\varphi_{i}
$$

Observação 2. Para qualquer $u \in D\left(\left(-\Delta_{N}\right)^{\frac{1}{2}}\right)$, temos que $\left(-\Delta_{N}\right)^{-\frac{1}{2}} u \in D\left(\left(-\Delta_{N}\right)^{\frac{1}{2}}\right)$.

De fato, por definição temos que

$$
D\left(\left(-\Delta_{N}\right)^{\frac{1}{2}}\right)=\left\{u \in X \quad \mid \quad\left(-\Delta_{N}\right)^{\frac{1}{2}} u \in X\right\} .
$$

Obviamente, $\left(-\Delta_{N}\right)^{-\frac{1}{2}} u \in X$, para qualquer $u \in X$, e por definição

$$
\left(-\Delta_{N}\right)^{-\frac{1}{2}}=\left(\left(-\Delta_{N}\right)^{\frac{1}{2}}\right)^{-1}
$$

no $D\left(\left(-\Delta_{N}\right)^{\frac{1}{2}}\right), \operatorname{logo}$

$$
\left(-\Delta_{N}\right)^{\frac{1}{2}}\left(\left(-\Delta_{N}\right)^{-\frac{1}{2}} u\right)=u \in X
$$

para qualquer $u \in D\left(\left(-\Delta_{N}\right)^{\frac{1}{2}}\right)$. Portanto, $\left(-\Delta_{N}\right)^{-\frac{1}{2}} u \in D\left(\left(-\Delta_{N}\right)^{\frac{1}{2}}\right)$, para qualquer $u \in D\left(\left(-\Delta_{N}\right)^{\frac{1}{2}}\right)$.

Sendo assim, como $\lambda_{i} \neq 0$, para qualquer $i \in \mathcal{I}$, temos por [27], que

$$
\left(-\Delta_{N}\right)^{\frac{1}{2}} u=\sum_{i \in \mathcal{I}}^{\infty} \lambda_{i}^{\frac{1}{2}}<u, \varphi_{i}>\varphi_{i}
$$


para qualquer $u \in D\left(\left(-\Delta_{N}\right)^{\frac{1}{2}}\right)$.

Tendo definido o operador $\left(-\Delta_{N}\right)^{\frac{1}{2}}$, vamos agora descrever seu domínio.

Proposição 9. Considere o conjunto

$$
Y=\left\{u \in X\left|\sum_{i \in \mathcal{I}}^{\infty} \lambda_{i}\right|<u, \varphi_{i}>\left.\right|^{2}<\infty\right\} .
$$

Então, temos que $D\left(\left(-\Delta_{N}\right)^{\frac{1}{2}}\right)=Y$.

Demonstração. Seja $u \in D\left(\left(-\Delta_{N}\right)^{\frac{1}{2}}\right), \operatorname{logo} u \in X$ e $\left(-\Delta_{N}\right)^{\frac{1}{2}} u \in X$, ou seja, a série

$$
\sum_{i \in \mathcal{I}}^{\infty} \lambda_{i}^{\frac{1}{2}}<u, \varphi_{i}>\varphi_{i}
$$

converge em $L^{2}(\Omega)$. Então, a série numérica

$$
\sum_{i \in \mathcal{I}}^{\infty} \lambda_{i}\left|<u, \varphi_{i}>\right|^{2}
$$

converge. Portanto, $D\left(\left(-\Delta_{N}\right)^{\frac{1}{2}}\right) \subset Y$.

Considere $v \in Y \subset X$, então a série numérica

$$
\sum_{i \in \mathcal{I}}^{\infty} \lambda_{i}\left|<v, \varphi_{i}>\right|^{2}
$$

é convergente, ou seja, a série

$$
\sum_{i \in \mathcal{I}}^{\infty} \lambda_{i}^{\frac{1}{2}}<v, \varphi_{i}>\varphi_{i}
$$

converge em $L^{2}(\Omega)$.

Sabemos que $\left(\varphi_{i}\right)_{\Omega}=0$, para qualquer $i \in \mathcal{I}$, logo, tomando

$$
s_{m}=\sum_{i \in \mathcal{I}}^{m} \lambda_{i}^{\frac{1}{2}}<v, \varphi_{i}>\varphi_{i}
$$

temos que

$$
\begin{aligned}
\left|\int_{\Omega}\left(-\Delta_{N}\right)^{\frac{1}{2}} v(x) d x\right| & =\left|\int_{\Omega}\left(-\Delta_{N}\right)^{\frac{1}{2}} v(x)-s_{m}(x) d x\right| \\
& \leq \int_{\Omega}\left|\left(-\Delta_{N}\right)^{\frac{1}{2}} v(x)-s_{m}(x)\right| d x \\
& \leq[\operatorname{med}(\Omega)]^{\frac{1}{2}}\left\|\left(-\Delta_{N}\right)^{\frac{1}{2}} v-s_{m}\right\|_{L^{2}(\Omega)} \stackrel{m \rightarrow \infty}{\longrightarrow 0 .} .
\end{aligned}
$$

Então,

$$
\int_{\Omega}\left(-\Delta_{N}\right)^{\frac{1}{2}} v(x) d x=0
$$


e assim $v \in D\left(\left(-\Delta_{N}\right)^{\frac{1}{2}}\right)$. Portanto, $Y=D\left(\left(-\Delta_{N}\right)^{\frac{1}{2}}\right)$.

Sendo assim, concluímos o seguinte teorema.

Teorema 5. Sejam $\left\{\varphi_{i}\right\}_{i \in \mathcal{I}}$ a base ortonormal de $X$ formada pelas autofunções do operador $-\Delta_{N} e$ $\left\{\lambda_{i}\right\}_{i \in \mathcal{I}}$ os respectivos autovalores, em $\Omega_{i}, \Omega_{q}$ ou $\Omega_{b}$. Então,

1.

$$
D\left(\left(-\Delta_{N}\right)^{\frac{1}{2}}\right)=\left\{u \in X\left|\sum_{i \in \mathcal{I}}^{\infty} \lambda_{i}\right|<u, \varphi_{i}>\left.\right|^{2}<\infty\right\} .
$$

2. O operador $\left(-\Delta_{N}\right)^{\frac{1}{2}}: D\left(\left(-\Delta_{N}\right)^{\frac{1}{2}}\right) \subset X \longrightarrow X$ é dado por

$$
\left(-\Delta_{N}\right)^{\frac{1}{2}} u=\sum_{i \in \mathcal{I}}^{\infty} \lambda_{i}^{\frac{1}{2}}<u, \varphi_{i}>\varphi_{i} .
$$




\section{Capítulo 4}

\section{O Operador}

Neste capítulo, temos por objetivo definir o operador $B_{\frac{1}{2}}$ e verificar que este operador coincide com a raiz quadrada do operador Laplaciano com condição de fronteira de Neumann $\left(-\Delta_{N}\right)^{\frac{1}{2}}$. Este operador $B_{\frac{1}{2}}$, será definido como uma extensão do operador $A_{\frac{1}{2}}$.

A definição do operador $A_{\frac{1}{2}}$ esta intimamente ligada com a existência e unicidade de solução clássica do seguinte problema de extensão harmônica

$$
\left\{\begin{array}{llll}
\Delta \tilde{v}(x, y, z) & =0 & \text { em } & \mathbb{R}_{+}^{3} \\
\tilde{v}(x, y, 0) & =\tilde{u}(x, y) & \text { em } & \mathbb{R}^{2} \\
\lim _{z \rightarrow \infty}\|\tilde{v}(\cdot, \cdot, z)\|_{L^{2}(\Omega)} & =0 & & \\
\lim _{z \rightarrow \infty}\left\|\tilde{v}_{z}(\cdot, \cdot, z)\right\|_{L^{2}(\Omega)} & =0 & \\
(\tilde{v}(\cdot, \cdot, z))_{\Omega} & =0 & \forall z \geq 0
\end{array}\right.
$$

onde $\tilde{u}$ é uma extensão que definiremos da função $u \in D\left(A_{\frac{1}{2}}\right)$ em $\mathbb{R}^{2}$,

$$
(\tilde{v}(\cdot, \cdot, z))_{\Omega}=\int_{\Omega} \tilde{v}(x, y, z) d x d y
$$

para qualquer $z \geq 0$ e o domínio $\Omega$ é $\Omega_{q}$ ou $\Omega_{b}$.

Quando o domínio for $\Omega_{i}$, o problema de extensão harmônica associado é

$$
\left\{\begin{array}{llll}
\Delta \tilde{v}(x, z) & =0 & \text { em } & \mathbb{R}_{+}^{2} \\
\tilde{v}(x, 0) & =\tilde{u}(x) & \text { em } \quad & \mathbb{R} \\
\lim _{z \rightarrow \infty}\|\tilde{v}(\cdot, z)\|_{L^{2}\left(\Omega_{i}\right)} & =0 & & \\
\lim _{z \rightarrow \infty}\left\|\tilde{v}_{z}(\cdot, z)\right\|_{L^{2}\left(\Omega_{i}\right)} & =0 & \\
(\tilde{v}(\cdot, z))_{\Omega_{i}} & =0 & \forall z \geq 0
\end{array}\right.
$$

onde $\tilde{u}$ é uma extensão que definiremos da função $u \in D\left(A_{\frac{1}{2}}\right)$ em $\mathbb{R}$,

$$
(\tilde{v}(\cdot, z))_{\Omega_{i}}=\int_{\Omega_{i}} \tilde{v}(x, z) d x
$$

para qualquer $z \geq 0$.

O capítulo será dividido em três seções, onde em cada seção o estudo será feito para cada caso do domínio $\Omega$. 


\subsection{Caso $\Omega_{i}=(0, \pi)$}

Seja $s>\frac{3}{2}$ e considere

$$
D\left(A_{\frac{1}{2}}\right)=\left\{u \in H^{s}\left(\Omega_{i}\right) \mid u_{x}(0)=u_{x}(\pi)=0 \text { e } \int_{\Omega_{i}} u(x) d x=0\right\} .
$$

Observe que

$$
u \in H^{s}\left(\Omega_{i}\right) \Rightarrow u_{x} \in H^{s-1}\left(\Omega_{i}\right) \hookrightarrow C\left(\Omega_{i}\right),
$$

pois $s-1>\frac{1}{2}$. Logo, a derivada de $u$ tem traço bem definido e com isso temos que $D\left(A_{\frac{1}{2}}\right)$ está bem definido.

Seja $u \in D\left(A_{\frac{1}{2}}\right)$ e considere a extensão par, $2 \pi$-periódica de $u$ em $\mathbb{R}$, isto é,

$$
\tilde{u}(x)=\left\{\begin{array}{ccccc}
u(x-2 k \pi) & \text { se } & 2 k \pi & \leq x \leq & (2 k+1) \pi \\
u(-x+2 k \pi) & \text { se } & (2 k-1) \pi<x & <x
\end{array},\right.
$$

com $k \in \mathbb{Z}$. Pela Proposição 14., temos que $\tilde{u} \in C(\mathbb{R})$.

Tendo estendido a função $u \in D\left(A_{\frac{1}{2}}\right)$ à $\mathbb{R}$, vejamos a definição de solução clássica de (4.2)

Definição 8. Dizemos que uma função $\tilde{v}: \mathbb{R}_{+}^{2} \longrightarrow \mathbb{R}$ é solução clássica de (4.2) se satisfaz

1. $\tilde{v} \in C_{\text {per }}^{2}\left(\mathbb{R}_{+}^{2}\right)$, isto é, $\tilde{v} \in C^{2}\left(\mathbb{R}_{+}^{2}\right)$ e ṽ é $2 \pi$-periódica na variável $x \in \mathbb{R}$.

2. $\tilde{v}$ é par na variável $x$.

3. $\tilde{v}$ satisfaz (4.2).

Usando o Método de Separação de Variáveis, temos que a função

$$
\tilde{v}(x, z)=\sum_{j=1}^{\infty} a_{j} e^{-j z} \cos (j x)
$$

onde

$$
a_{j}=\frac{2}{\pi} \int_{0}^{\pi} u(x) \cos (j x) d x
$$

para qualquer $j \geq 1$, é candidata à solução de (4.2). O próximo teorema, verifica que de fato esta função é a única solução de (4.2).

Teorema 6. Seja $u \in D\left(A_{\frac{1}{2}}\right)$ e ú sua extensão par, $2 \pi$-periódica em $\mathbb{R}$. Então, a função $\tilde{v}: \mathbb{R}_{+}^{2} \longrightarrow \mathbb{R}$ dada por (4.3) e (4.4) é a única solução clássica de (4.2).

Demonstração. Primeiramente, temos que $\tilde{v}$ é uma função par, $2 \pi$-periódica na variável $x$, devido a paridade e periodicidade da função cosseno.

1. $\tilde{v} \in C_{\text {per }}\left(\mathbb{R}_{+}^{2}\right)$.

De fato, observe que

$$
\left|a_{j} e^{-j z} \cos (j x)\right| \leq \frac{2}{\pi^{2}}\left|<u, \varphi_{j}>\right|^{2}+\frac{1}{2} e^{-2 j z},
$$


onde $\varphi_{j}(x)=\sqrt{\frac{2}{\pi}} \cos (j x)$.

Sabemos que a desigualdade

$$
j^{n} e^{-2 j z} \leq \frac{C}{j^{2}}
$$

é válida para qualquer $n \in \mathbb{Z}_{+}$e para cada $z>0$, onde $C$ é uma constante que depende de $z$. Logo,

$$
\left|a_{j} e^{-j z} \cos (j x)\right| \leq \frac{2}{\pi^{2}}\left|<u, \varphi_{j}>\right|^{2}+\frac{C}{j^{2}},
$$

para cada $(x, z) \in \mathbb{R}_{+}^{2}$ e para qualquer $j \geq 1$.

Então, usando a Identidade de Parseval, temos que as séries numéricas

$$
\sum_{j=1}^{\infty}\left|<u, \varphi_{j}>\right|^{2} \text { e } \sum_{j=1}^{\infty} \frac{C}{j^{2}}
$$

convergem, com isso pelo Critério de Weierstrass temos que $\tilde{v}$ é contínua em $[0, \pi] \times(0, \infty)$.

Portanto, pela periodicidade e paridade de $\tilde{v}$ na variável $x$, segue que $\tilde{v} \in C_{p e r}\left(\mathbb{R}_{+}^{2}\right)$.

2. $\tilde{v} \in C_{\text {per }}^{2}\left(\mathbb{R}_{+}^{2}\right)$.

De fato, segue pelos mesmos argumentos do item anterior, usando o fato que

$$
\tilde{v}_{x}(0, z)=\tilde{v}_{x}(\pi, z)=0
$$

para qualquer $z>0$.

3. $\tilde{v}$ satisfaz (4.2).

De fato, pela convergência pontual da série que define $\tilde{v}$ e a convergência em compactos da série das derivadas, fazendo alguns cálculos, vemos que

$$
\Delta \tilde{v}(x, z)=0,
$$

para qualquer $(x, z) \in \mathbb{R}_{+}^{2}$.

Seja $u \in D\left(A_{\frac{1}{2}}\right) \subset X$, temos que

$$
u=\sum_{j \geq 1}^{\infty}<u, \varphi_{j}>\varphi_{j}
$$

em $L^{2}\left(\Omega_{i}\right)$, onde $\varphi_{j}$ são as autofunções de $-\Delta_{N}$ em $\Omega_{i}$. Logo,

$$
\tilde{u}=\sum_{j \geq 1}^{\infty}<u, \varphi_{j}>\varphi_{j}
$$

em $L_{\text {per }}^{2}(\mathbb{R})$ 
Considerando a sequência $\left\{\psi_{m}\right\} \subset C_{\text {per }}^{\infty}\left(\overline{\mathbb{R}_{+}^{2}}\right)$ tal que

$$
\psi_{m}(x, z)=\sum_{j \geq 1}^{m} a_{j} e^{-j z} \cos (j x)
$$

temos que

$$
\lim _{m \rightarrow \infty}\left\|\tilde{v}-\psi_{m}\right\|_{H_{p e r}^{1}\left(\mathbb{R}_{+}^{2}\right)}=0 .
$$

Então, concluímos que $\tilde{v} \in H_{\text {per }}^{1}\left(\mathbb{R}_{+}^{2}\right)$. Pelo Teorema do Traço, segue que

$$
\tilde{v}(\cdot, 0)=\sum_{j \geq 1}^{\infty}<u, \varphi_{j}>\varphi_{j}
$$

em $L_{\text {per }}^{2}(\mathbb{R})$. Portanto, $\tilde{v}(\cdot, 0)=\tilde{u}(\cdot)$ quase sempre em $\mathbb{R}$.

Por outro lado,

$$
\|\tilde{v}(\cdot, z)\|_{L^{2}\left(\Omega_{i}\right)}^{2} \leq \pi \sum_{j=1}^{\infty}\left|a_{j}\right|^{2} e^{-2 j z} \stackrel{z \rightarrow \infty}{\longrightarrow} 0
$$

analogamente, temos que

$$
\left\|\tilde{v}_{z}(\cdot, z)\right\|_{L^{2}\left(\Omega_{i}\right)} \stackrel{z \rightarrow \infty}{\longrightarrow} 0 .
$$

Usando também as propriedades de convergência uniforme de série de funções, segue que

$$
\int_{0}^{\pi} \tilde{v}(x, z) d x=\sum_{j=1}^{\infty} a_{j} e^{-j z}\left(\int_{0}^{\pi} \cos (j x) d x\right)=0,
$$

para qualquer $z>0$. O mesmo é válido para $z=0$, já que $\tilde{u}$ tem média zero e coincide quase sempre $\operatorname{com} \tilde{v}(\cdot, 0)$ em $\mathbb{R}$.

Portanto, $\tilde{v}$ é solução clássica de (4.2). Para provarmos a unicidade de solução clássica suponha que $\tilde{v}_{1}, \tilde{v}_{2}$ sejam soluções clássicas (4.2), então $\tilde{w}=\tilde{v}_{1}-\tilde{v}_{2}$ é solução clássica de

$$
\left\{\begin{array}{lll}
\Delta \tilde{w}(x, z)=0 & \text { em } & \mathbb{R}_{+}^{2} \\
\tilde{w}(x, 0)=0 & \text { em } & \mathbb{R} \\
\lim _{z \rightarrow \infty}\|\tilde{w}(\cdot, z)\|_{L^{2}\left(\Omega_{i}\right)}=0 & & \\
\lim _{z \rightarrow \infty}\left\|\tilde{w}_{z}(\cdot, z)\right\|_{L^{2}\left(\Omega_{i}\right)}=0 & & \\
(\tilde{w}(\cdot, z))_{\Omega_{i}}=0 & \forall z \geq 0 &
\end{array} .\right.
$$

Sabemos também que $\tilde{w}$ é par na variável $x$ e $\tilde{w} \in H_{\text {per }}^{1}\left(\mathbb{R}_{+}^{2}\right)$, já que $\tilde{v_{1}}, \tilde{v_{2}} \in H_{\text {per }}^{1}\left(\mathbb{R}_{+}^{2}\right)$.

Considere o espaço de Hilbert $\mathcal{H}$ como sendo o conjunto das funções $u \in H_{\text {per }}^{1}\left(\mathbb{R}_{+}^{2}\right)$ satisfazendo

1. $u$ é par quase sempre na variável $x$.

2. $(u(\cdot, z))_{\Omega_{i}}=0$, para qualquer $z \geq 0$.

3. $u(\cdot, 0)=0$ quase sempre em $\mathbb{R}$. 
4. $\lim _{z \rightarrow \infty}\|u(\cdot, z)\|_{L^{2}\left(\Omega_{i}\right)}=\lim _{z \rightarrow \infty}\left\|u_{z}(\cdot, z)\right\|_{L^{2}\left(\Omega_{i}\right)}=0$.

com norma dada por

$$
\|u\|_{\mathcal{H}}=\left(\int_{0}^{\infty} \int_{0}^{\pi}|\nabla u(x, z)|^{2} d x d z\right)^{\frac{1}{2}} .
$$

Usando o Método de Integração por Partes, temos que $\tilde{w}$ satisfaz

$$
\int_{0}^{\infty} \int_{0}^{\pi} \nabla \tilde{w}(x, z) \nabla \tilde{\varphi}(x, z) d x d z=0
$$

para qualquer $\tilde{\varphi} \in \mathcal{H}$.

Por outro lado, considere o funcional $h: \mathcal{H} \times \mathcal{H} \longrightarrow \mathbb{R}$ tal que

$$
h(\tilde{\psi}, \tilde{\varphi})=\int_{0}^{\infty} \int_{0}^{\pi} \nabla \tilde{\psi}(x, z) \nabla \tilde{\varphi}(x, z) d x d z
$$

para qualquer $\tilde{\psi}, \tilde{\varphi} \in \mathcal{H}$.

Verificamos facilmente, que este funcional $h$ satisfaz as hipóteses do Teorema de Lax-Milgran, então existe uma única função $\tilde{\psi} \in \mathcal{H}$, tal que

$$
h(\tilde{\psi}, \tilde{\varphi})=0
$$

para qualquer $\tilde{\varphi} \in \mathcal{H}$.

Como as funções nula e $\tilde{w}$ satisfazem (4.5), segue que $\tilde{w}=0$ e portanto $\tilde{v}_{1}=\tilde{v}_{2}$.

Através da existência e unicidade de solução clássica do problema de extensão harmônica (4.2), temos a seguinte definição.

Definição 9. Seja

$$
D\left(A_{\frac{1}{2}}\right)=\left\{u \in H^{s}\left(\Omega_{i}\right) \mid s>\frac{3}{2}, \quad u_{x}(0)=u_{x}(\pi)=0 e \int_{\Omega_{i}} u(x) d x=0\right\} .
$$

Definimos o seguinte operador

$$
\begin{aligned}
A_{\frac{1}{2}}: D\left(A_{\frac{1}{2}}\right) \subset X & \longrightarrow X \\
u & \longmapsto-\left.\left(\tilde{v}_{z}(\cdot, 0)\right)\right|_{\Omega_{i}},
\end{aligned}
$$

onde ṽ é a única solução clássica (4.2).

Observação 3. Vejamos que o operador $A_{\frac{1}{2}}$ está bem definido. Isto ocorre, devido a unicidade de solução do problema (4.2) e também pelo fato de que $A_{\frac{1}{2}} \in X$, pois

$$
\tilde{v}_{z}(\cdot, 0)=-\sum_{j=1}^{\infty} \lambda_{j}^{\frac{1}{2}}<u, \varphi_{j}>\varphi_{j},
$$

em $L^{2}\left(\Omega_{i}\right)$ 
Pela observação anterior temos que o operador $A_{\frac{1}{2}}$ pode ser visto como uma série envolvendo as autofunções e os respectivos autovalores do operador $-\Delta_{N}$ em $\Omega_{i}$. Isso decorre do fato dos autovalores do operador $-\Delta_{N}$ em $\Omega_{i}$ serem dados por $\lambda_{j}=j^{2}$, para qualquer $j \geq 1$, e

$$
a_{j}=\frac{2}{\pi} \int_{\Omega_{i}} u(x) \cos (j x) d x=\sqrt{\frac{2}{\pi}} \int_{\Omega_{i}} u(x) \varphi_{j}(x) d x
$$

onde $\varphi_{j}(x)=\sqrt{\frac{2}{\pi}} \cos (j x)$, para qualquer $j \geq 1$.

Logo,

$$
A_{\frac{1}{2}} u=\sum_{j=1}^{\infty} \lambda_{j}^{\frac{1}{2}}<u, \varphi_{j}>\varphi_{j},
$$

em $L^{2}\left(\Omega_{i}\right)$.

Nosso objetivo agora, após ter definido o operador $A_{\frac{1}{2}}$, é definir o operador $B_{\frac{1}{2}}$.

Definição 10. Seja

$$
Y=\left\{u \in X\left|\sum_{j=1}^{\infty} \lambda_{j}\right|<u, \varphi_{j}>\left.\right|^{2}<\infty\right\} .
$$

Definimos o operador

$$
\begin{aligned}
B_{\frac{1}{2}}: Y \subset X & \longrightarrow X \\
u & \longmapsto \sum_{j=1}^{\infty} \lambda_{j}^{\frac{1}{2}}<u, \varphi_{j}>\varphi_{j},
\end{aligned}
$$

onde $\lambda_{j}=j^{2}$ e $\varphi_{j}(x)=\sqrt{\frac{2}{\pi}} \cos (j x)$, para qualquer $j \geq 1$.

O operador $B_{\frac{1}{2}}$ é definido por meio da convergência na norma de $X$, isto é,

$$
\lim _{m \rightarrow \infty}\left\|B_{\frac{1}{2}} u-\sum_{j=1}^{m} \lambda_{j}^{\frac{1}{2}}<u, \varphi_{j}>\varphi_{j}\right\|=0 .
$$

Lema 6. O operador $B_{\frac{1}{2}}$ está bem definido e é uma extensão do operador $A_{\frac{1}{2}}$ a $Y$.

Demonstração. Seja $u \in Y$, logo a série

$$
\sum_{j=1}^{\infty} \lambda_{j}^{\frac{1}{2}}<u, \varphi_{j}>\varphi_{j}
$$

converge em $L^{2}\left(\Omega_{i}\right)$, ou seja, $B_{\frac{1}{2}} u \in L^{2}\left(\Omega_{i}\right)$.

Sabemos que

$$
\int_{\Omega_{i}} \varphi_{j}(x) d x=0
$$

para qualquer $j \geq 1$. 
Logo, considerando as somas parciais, dadas por

$$
s_{m}=\sum_{j=1}^{m} \lambda_{j}^{\frac{1}{2}}<u, \varphi_{j}>\varphi_{j},
$$

temos que

$$
\begin{aligned}
\left|\int_{\Omega_{i}} B_{\frac{1}{2}} u(x) d x\right| & \leq \int_{\Omega_{i}}\left|B_{\frac{1}{2}} u(x)-s_{m}(x)\right| d x \\
& \leq \pi^{\frac{1}{2}}\left\|B_{\frac{1}{2}} u-s_{m}\right\| \stackrel{m \rightarrow \infty}{\longrightarrow} 0
\end{aligned}
$$

ou seja,

$$
\int_{\Omega_{i}} B_{\frac{1}{2}} u(x) d x=0
$$

e assim $B_{\frac{1}{2}} u \in X$. Então, devido a unicidade da Série de Fourier, o operador está bem definido.

Seja $u \in D\left(A_{\frac{1}{2}}\right)$, logo $A_{\frac{1}{2}} u \in X$ e assim temos que

$$
\sum_{j=1}^{\infty}\left|<A_{\frac{1}{2}} u, \varphi_{j}>\right|^{2}=\sum_{j=1}^{\infty} \lambda_{j}\left|<u, \varphi_{j}>\right|^{2}<\infty .
$$

Então, segue que $D\left(A_{\frac{1}{2}}\right) \subset Y$ e ainda

$$
\begin{aligned}
\left\|A_{\frac{1}{2}} u-B_{\frac{1}{2}} u\right\|_{L^{1}\left(\Omega_{i}\right)} & \leq \int_{\Omega_{i}}\left|A_{\frac{1}{2}} u(x)-\sum_{j=1}^{m} \lambda_{j}^{\frac{1}{2}}<u, \varphi_{j}>\varphi_{j}(x)\right| d x \\
& +\int_{\Omega_{i}}\left|\sum_{j=1}^{m} \lambda_{j}^{\frac{1}{2}}<u, \varphi_{j}>\varphi_{j}(x)-B_{\frac{1}{2}} u(x)\right| d x \\
& \leq \pi^{\frac{1}{2}}\left\|A_{\frac{1}{2}} u-\sum_{j=1}^{m} \lambda_{j}^{\frac{1}{2}}<u, \varphi_{j}>\varphi_{j}\right\| \\
& +\pi^{\frac{1}{2}}\left\|B_{\frac{1}{2}} u-\sum_{j=1}^{m} \lambda_{j}^{\frac{1}{2}}<u, \varphi_{j}>\varphi_{j}\right\| .
\end{aligned}
$$

Sabemos, por definição do operador $B_{\frac{1}{2}}$, que

$$
\left\|B_{\frac{1}{2}} u-\sum_{j=1}^{m} \lambda_{j}^{\frac{1}{2}}<u, \varphi_{j}>\varphi_{j}\right\| . \stackrel{m \rightarrow \infty}{\longrightarrow} 0 .
$$

Por outro lado, considerando a ortonormalidade das autofunções e a Identidade de Parseval, temos que

$$
\begin{aligned}
\sum_{j=1}^{m} \lambda_{j}^{\frac{1}{2}}<u, \varphi_{j}>\varphi_{j} & =\sum_{j=1}^{m}<A_{\frac{1}{2}} u, \varphi_{j}>\varphi_{j}, \\
\left\|A_{\frac{1}{2}} u\right\|^{2} & =\sum_{j=1}^{\infty}\left|<A_{\frac{1}{2}} u, \varphi_{j}>\right|^{2} .
\end{aligned}
$$


Então, pela Identidade de Pitágoras segue que

$$
\left\|A_{\frac{1}{2}} u-\sum_{j=1}^{m} \lambda_{j}^{\frac{1}{2}}<u, \varphi_{j}>\varphi_{j}\right\|^{2}=\left\|A_{\frac{1}{2}} u\right\|^{2}-\sum_{j=1}^{m}\left|<A_{\frac{1}{2}} u, \varphi_{j}>\right|^{2},
$$

para qualquer $m \in \mathbb{N}^{*}$, logo

$$
\left\|A_{\frac{1}{2}} u-\sum_{j=1}^{m} \lambda_{j}^{\frac{1}{2}}<u, \varphi_{j}>\varphi_{j}\right\| \stackrel{m \rightarrow \infty}{\longrightarrow} 0 .
$$

Portanto,

$$
A_{\frac{1}{2}} u=B_{\frac{1}{2}} u
$$

quase sempre em $\Omega_{i}$, para qualquer $u \in D\left(A_{\frac{1}{2}}\right)$, e assim $B_{\frac{1}{2}}$ é uma extensão de $A_{\frac{1}{2}}$ a $Y$.

Finalmente, concluiremos esta seção, provando que o operador $B_{\frac{1}{2}}$ é de fato o operador $\left(-\Delta_{N}\right)^{\frac{1}{2}}$. Para isso, consideraremos o operador Laplaciano com condição de fronteira de Neumann, como uma série formada pelas suas autofunções e autovalores, ou seja,

$$
-\Delta_{N} u=\sum_{j=1}^{\infty} \lambda_{j}<u, \varphi_{j}>\varphi_{j}
$$

para qualquer $u \in D\left(-\Delta_{N}\right)$. Observe que, para $u \in D\left(-\Delta_{N}\right)$, a série numérica

$$
\sum_{j=0}^{\infty} \lambda_{j}^{2}\left|<u, \varphi_{j}>\right|^{2}
$$

é convergente.

Com isso provaremos o seguinte teorema.

Teorema 7. O operador $B_{\frac{1}{2}}$ coincide com o operador $\left(-\Delta_{N}\right)^{\frac{1}{2}}$ em $\Omega_{i}$, ou seja,

$$
<u, B_{\frac{1}{2}} u>\geq 0
$$

para qualquer $u \in D\left(B_{\frac{1}{2}}\right) e$

$$
B_{\frac{1}{2}} \circ B_{\frac{1}{2}} u=-\Delta_{N} u
$$

para qualquer $u \in D\left(-\Delta_{N}\right)$.

Demonstração. Seja $u \in D\left(-\Delta_{N}\right) \subset X$. Como $\lambda_{j}=j^{2} \geq 1$, para qualquer $j \geq 1$, segue que

$$
\sum_{j=1}^{\infty} \lambda_{j}\left|<u, \varphi_{j}>\right|^{2} \leq \sum_{j=1}^{\infty} \lambda_{j}^{2}\left|<u, \varphi_{j}>\right|^{2}<\infty
$$


Logo, $D\left(-\Delta_{N}\right) \subset D\left(B_{\frac{1}{2}}\right)=Y$ e assim

$$
B_{\frac{1}{2}} u=\sum_{j=1}^{\infty} \lambda_{j}^{\frac{1}{2}}<u, \varphi_{j}>\varphi_{j}
$$

Também temos que $B_{\frac{1}{2}} u \in D\left(B_{\frac{1}{2}}\right)$, pois $B_{\frac{1}{2}} u \in X$ e

$$
\sum_{j=1}^{\infty} \lambda_{j}\left|<B_{\frac{1}{2}} u, \varphi_{j}>\right|^{2}=\sum_{j=1}^{\infty} \lambda_{j}^{2}\left|<u, \varphi_{j}>\right|^{2}<\infty .
$$

Então, pela ortonormalidade das autofunções, segue que

$$
\begin{aligned}
B_{\frac{1}{2}} \circ B_{\frac{1}{2}} u & =\sum_{j=1}^{\infty} \lambda_{j}^{\frac{1}{2}}<B_{\frac{1}{2}} u, \varphi_{j}>\varphi_{j} \\
& =\sum_{j=1}^{\infty} \lambda_{j}^{\frac{1}{2}}\left\langle\sum_{k=1}^{\infty} \lambda_{k}^{\frac{1}{2}}<u, \varphi_{k}>\varphi_{k}, \varphi_{j}\right\rangle \varphi_{j} \\
& =\sum_{j=1}^{\infty} \sum_{k=1}^{\infty} \lambda_{j}^{\frac{1}{2}} \lambda_{k}^{\frac{1}{2}}<u, \varphi_{k}><\varphi_{k}, \varphi_{j}>\varphi_{j} \\
& =\sum_{j=1}^{\infty} \lambda_{j}<u, \varphi_{k}>\varphi_{j} .
\end{aligned}
$$

Portanto,

$$
B_{\frac{1}{2}} \circ B_{\frac{1}{2}} u=-\Delta_{N} u
$$

para qualquer $u \in D\left(-\Delta_{N}\right)$.

Temos ainda para $u \in D\left(B_{\frac{1}{2}}\right)$ que

$$
<u, B_{\frac{1}{2}} u>=\sum_{j=1}^{\infty} \lambda_{j}^{\frac{1}{2}}\left|<u, \varphi_{j}>\right|^{2} \geq 0,
$$

portanto $B_{\frac{1}{2}} u$ coincide com o operador $\left(-\Delta_{N}\right)^{\frac{1}{2}}$.

\subsection{Caso $\Omega_{q}=(0, \pi) \times(0, \pi)$}

Seja $s>2$ e considere

$$
D\left(A_{\frac{1}{2}}\right)=\left\{u \in H^{s}\left(\Omega_{q}\right)\left|\frac{\partial u}{\partial n}\right|_{\partial \Omega_{q}}=0 \text { e } \int_{\Omega_{q}} u(x, y) d x d y=0\right\} .
$$

Observe que

$$
u \in H^{s}\left(\Omega_{q}\right) \Rightarrow \frac{\partial u}{\partial n} \in H^{s-1}\left(\Omega_{q}\right) \hookrightarrow C\left(\Omega_{q}\right),
$$

pela Proposição 1., pois $s-1>1$. Logo, a derivada de $u$ tem traço bem definido e com isso temos que $D\left(A_{\frac{1}{2}}\right)$ está bem definido. 
Seja $u \in D\left(A_{\frac{1}{2}}\right)$ e considere a extensão par, $2 \pi$-periódica nas variáveis $x$ e $y$ de $u$ em $\mathbb{R}^{2}$, isto é, a função $\tilde{u}: \mathbb{R}^{2} \longrightarrow \mathbb{R}$ tal que

$$
\tilde{u}(x, y)=\left\{\begin{array}{lll}
u(x-2 t \pi, y-2 k \pi) & \text { se } & (x, y) \in[2 t \pi,(2 t+1) \pi] \times[2 k \pi,(2 k+1) \pi] \\
u(-x+2 t \pi,-y+2 k \pi) & \text { se } & (x, y) \in[(2 t-1) \pi, 2 t \pi] \times[(2 k-1) \pi, 2 k \pi] \\
u(-x+2 t \pi, y-2 k \pi) & \text { se } & (x, y) \in[(2 t-1) \pi, 2 t \pi] \times[2 k \pi,(2 k+1) \pi] \\
u(x-2 t \pi,-y+2 k \pi) & \text { se } & (x, y) \in[2 t \pi,(2 t+1) \pi] \times[(2 k-1) \pi, 2 k \pi]
\end{array},\right.
$$

$\operatorname{com} t, k \in \mathbb{Z}$.

Lema 7. A função ũ definida em (4.6), satisfaz as seguintes propriedades

1. ú é periódica nas variáveis $x$ e $y$, isto é,

$$
\tilde{u}(x+2 m \pi, y+2 n \pi)=\tilde{u}(x, y)
$$

para qualquer $(x, y) \in \mathbb{R}^{2}$ e $m, n \in \mathbb{Z}$.

2. ú é par nas variáveis $x$ e $y$, isto é,

$$
\tilde{u}(-x, y)=\tilde{u}(x,-y)=\tilde{u}(x, y)
$$

para qualquer $(x, y) \in \mathbb{R}^{2}$.

3. $\tilde{u} \in C_{\text {per }}\left(\mathbb{R}^{2}\right)$.

Demonstração. Seja $y \in \mathbb{R}$ e considere a função $\tilde{u}^{y}: \mathbb{R} \longrightarrow \mathbb{R}$ tal que

$$
\tilde{u}^{y}(x)=\tilde{u}(x, y)
$$

Logo, pela Proposição 14., temos que $\tilde{u}^{y}$ é uma extensão par, $2 \pi$-periódica da função $u(\cdot, y)$ e $\tilde{u}^{y} \in C_{\text {per }}(\mathbb{R})$, para cada $y \in \mathbb{R}$ fixado.

Analogamente, fixando $x \in \mathbb{R}$ temos que a função $\tilde{u}^{x}: \mathbb{R} \longrightarrow \mathbb{R}$ tal que

$$
\tilde{u}^{x}(y)=\tilde{u}(x, y)
$$

é uma extensão par, $2 \pi$-periódica de $u(x, \cdot)$ e $\tilde{u}^{x} \in C_{\text {per }}(\mathbb{R})$, para cada $x \in \mathbb{R}$ fixado.

Com isso, temos que a função $\tilde{u}$ é $2 \pi$-periódica nas variáveis $x$ e $y$, e satisfaz

$$
\tilde{u}(-x, y)=\tilde{u}(x,-y)=\tilde{u}(x, y)
$$

para qualquer $x, y \in \mathbb{R}$.

Agora mostremos que $\tilde{u} \in C_{\text {per }}\left(\mathbb{R}^{2}\right)$. Como $u \in C\left(\Omega_{q}\right)$, pela periodicidade e paridadde da função $\tilde{u}$, temos que $\tilde{u}$ é contínua em $\mathbb{R}$, exceto possivelmente, nos pontos $\{k \pi\} \times[0, \pi]$ e $[0, \pi] \times\{k \pi\}$, para qualquer $k \in \mathbb{Z}$. Para verificarmos a continuidade de $\tilde{u}$ nestes pontos, basta verificarmos a continuidade de $\tilde{u}$ nos pontos $\{0\} \times[0, \pi],\{\pi\} \times[0, \pi],[0, \pi] \times\{0\}$ e $[0, \pi] \times\{\pi\}$, isso pela periodicidade e paridade de $\tilde{u}$. 
Seja $\left(0, y_{0}\right) \in\{0\} \times[0, \pi]$ arbitrário. Sabemos que $\tilde{u}^{0}$ e $\tilde{u}^{y}$ são contínuas em $y_{0}$ e 0 , respectivamente, para qualquer $y \in \mathbb{R}$. Logo, dado $\epsilon>0$, existem $\tilde{\delta}, \bar{\delta}>0$, tais que

$$
\begin{aligned}
|x|<\tilde{\delta} & \Longrightarrow\left|\tilde{u}^{y}(x)-\tilde{u}^{y}(0)\right|=|\tilde{u}(x, y)-\tilde{u}(0, y)|<\frac{\epsilon}{2} \\
\left|y-y_{0}\right|<\bar{\delta} & \Longrightarrow\left|\tilde{u}^{0}(y)-\tilde{u}^{0}\left(y_{0}\right)\right|=\left|\tilde{u}(0, y)-\tilde{u}\left(0, y_{0}\right)\right|<\frac{\epsilon}{2}
\end{aligned}
$$

Então, tomando $\delta=\min \{\tilde{\delta}, \bar{\delta}\}$, temos que

$$
\left|(x, y)-\left(0, y_{0}\right)\right|<\delta \Longrightarrow\left|\tilde{u}(x, y)-\tilde{u}\left(0, y_{0}\right)\right|<\epsilon,
$$

ou seja, $\tilde{u}$ é contínua em $\left(0, y_{0}\right)$, para qualquer $y_{0} \in[0, \pi]$.

Usando a mesma idéia, verificamos a continuidade de $\tilde{u} \operatorname{nos}$ pontos $\{\pi\} \times[0, \pi],[0, \pi] \times\{0\}$ e $[0, \pi] \times\{\pi\}$. Portanto, $\tilde{u} \in C_{\text {per }}\left(\mathbb{R}^{2}\right)$.

Queremos verificar a existência e unicidade de solução clássica do problema (4.1). Para isto, vejamos a definição de solução clássica.

Definição 11. Dizemos que uma função $\tilde{v}: \mathbb{R}_{+}^{3} \longrightarrow \mathbb{R}$ é solução de (4.1) se satisfaz

1. $\tilde{v} \in C_{p e r}^{2}\left(\mathbb{R}_{+}^{3}\right)$.

2. $\tilde{v}$ é par nas variáveis $x$ e $y$.

3. $\tilde{v}$ satisfaz (4.1).

Considere o seguinte problema

$$
\left\{\begin{array}{lll}
\Delta \tilde{v}(x, y, z)=0 & \text { em } & \Omega_{q} \times(0, \infty) \\
\tilde{v}(-x, y, z)=\tilde{v}(x,-y, z)=\tilde{v}(x, y, z) & \text { em } & \mathbb{R}^{2} \times[0, \infty) \\
\tilde{v}(x+2 \pi, y+2 \pi, z)=\tilde{v}(x, y, z) & \text { em } & \mathbb{R}^{2} \times[0, \infty) \\
\tilde{v}(x, y, 0)=\tilde{u}(x, y) & \text { em } & \mathbb{R}^{2} \\
\lim _{z \rightarrow \infty}\|\tilde{v}(\cdot, \cdot, z)\|_{L^{2}\left(\Omega_{q}\right)}=0 & & \\
\lim _{z \rightarrow \infty}\left\|\tilde{v}_{z}(\cdot, \cdot, z)\right\|_{L^{2}\left(\Omega_{q}\right)}=0 & & \\
(\tilde{v}(\cdot, \cdot, z))_{\Omega_{q}}=0 & \forall z \geq 0
\end{array} .\right.
$$

Usando separação de variáveis, procuramos soluções da forma $\tilde{v}(x, y, z)=X(x) Y(y) Z(z)$. Logo, temos os seguintes problemas

$$
\begin{gathered}
\left\{\begin{array}{ll}
X^{\prime \prime}(x)=\beta X(x) & \text { em }(0, \pi) \\
X(-x)=X(x) & \text { em } \mathbb{R} \\
X(x+2 \pi)=X(x) & \text { em } \quad \mathbb{R}
\end{array}, \begin{cases}Y^{\prime \prime}(y)=-(\beta-\theta) Y(y) & \text { em }(0, \pi) \\
Y(-y)=Y(y) & \text { em } \mathbb{R} \\
Y(y+2 \pi)=Y(y) & \text { em } \mathbb{R}\end{cases} \right. \\
\left\{\begin{array}{l}
Z^{\prime \prime}(z)=-\theta Z(z) \text { em }(0, \infty) \\
\lim _{z \rightarrow \infty} Z(z)=\lim _{z \rightarrow \infty} Z^{\prime}(z)=0
\end{array}\right.
\end{gathered}
$$

onde $\beta, \theta \in \mathbb{R}$. 
Fazendo algumas contas, verificamos que as soluções gerais destes problemas são dadas formalmente por

$$
X_{j}(x)=A_{j} \cos (j x), \quad Y_{j k}(y)=C_{j k} \cos (k y), \quad Z_{j k}(z)=D_{j k} e^{-\sqrt{k^{2}+j^{2}} z},
$$

onde $A_{j}, C_{j k}, D_{j k}$ são constantes a determinar, para qualquer $j, k \in \mathbb{Z}_{+}$.

Tome

$$
\tilde{v}_{j k}(x, y, z)=\bar{A}_{j k} e^{-\sqrt{k^{2}+j^{2}} z} \cos (j x) \cos (k y)
$$

e impondo a condição

$$
\int_{\Omega_{q}} \tilde{v}(x, y, z) d x d y=0
$$

para qualquer $z \geq 0$, vemos que $\bar{A}_{00}=0$.

Então, considere

$$
\tilde{v}(x, y, z)=\sum_{(j, k) \in \mathcal{I}}^{\infty} \bar{A}_{j k} e^{-\sqrt{k^{2}+j^{2}} z} \cos (j x) \cos (k y) .
$$

Queremos ainda que

$$
\tilde{u}(x, y)=\tilde{v}(x, y, 0)=\sum_{(j, k) \in \mathcal{I}}^{\infty} \bar{A}_{j k} \cos (j x) \cos (k y)
$$

quase sempre em $\mathbb{R}^{2}$, logo basta tomarmos $\bar{A}_{j k}$ como os coeficientes de Fourier da série de Fourier de $\tilde{u}$.

Portanto, uma candidata a solução de (4.1) em $\Omega_{q}$ é dada por

$$
\tilde{v}(x, y, z)=\sum_{(j, k) \in \mathcal{I}}^{\infty} \bar{A}_{j k} e^{-\sqrt{k^{2}+j^{2}} z} \cos (j x) \cos (k y) .
$$

O próximo teorema, verifica que esta função $\tilde{v}$ é de fato solução do problema (4.1) em $\Omega_{q}$.

Teorema 8. Seja $u \in D\left(A_{\frac{1}{2}}\right)$ e ũ sua extensão par, $2 \pi$-periódica, nas variáveis $x$ e y, em $\mathbb{R}^{2}$. Então, a função $\tilde{v}: \mathbb{R}_{+}^{3} \longrightarrow \mathbb{R}$ tal que

$$
\tilde{v}(x, y, z)=\sum_{(j, k) \in \mathcal{I}}^{\infty} \bar{A}_{j k} e^{-\sqrt{k^{2}+j^{2}} z} \cos (j x) \cos (k y),
$$

onde

$$
\bar{A}_{j k}=\beta_{j k}^{2} \int_{\Omega_{q}} u(x, y) \cos (j x) \cos (k y) d x d y
$$

tal que

$$
\beta_{j k}=\left\{\begin{array}{l}
\frac{\sqrt{2}}{\pi} \quad \text { se } \quad k=0 \text { ou } j=0 \\
\frac{2}{\pi} \quad \text { se } j, k \geq 1
\end{array} .\right.
$$

Demonstração. Obviamente temos que a função $\tilde{v}$ é par e $2 \pi$-periódica nas variáveis $x$ e $y$, pelo fato da função cosseno satisfazer estas propriedades. 
Para verificar que $\tilde{v} \in C_{\text {per }}^{2}\left(\mathbb{R}_{+}^{3}\right)$, vamos utilizar o Teste de Weierstrass de [30] e o fato de que uma série de funções contínuas que converge uniformemente convergirá para uma função contínua, para o caso de séries como somabilidade de famílias indexadas por multi-índices.

1. $\tilde{v} \in C_{\text {per }}\left(\mathbb{R}_{+}^{3}\right)$.

De fato, observe que

$$
\left|\bar{A}_{j k} e^{-\sqrt{j^{2}+k^{2}} z} \cos (j x) \cos (k y)\right| \leq \frac{\left|\bar{A}_{j k}\right|^{2}}{2}+\frac{1}{2} e^{-2 \sqrt{j^{2}+k^{2}} z},
$$

para qualquer $j, k \geq 1$.

Mas,

$$
e^{-2 \sqrt{j^{2}+k^{2}} z} \leq \frac{C}{\left(j^{2}+k^{2}\right)^{2}}
$$

para qualquer $(j, k) \in \mathcal{I}$ e para cada $z>0$, onde $C$ é uma constante que depende de $z$ e

$$
\left|\bar{A}_{j k}\right|^{2}=\beta_{j k}^{2}\left|<u, \varphi_{j k}>\right|^{2}
$$

tal que $\varphi_{j k}(x, y)=\beta_{j k} \cos (j x) \cos (k y)$, para qualquer $(j, k) \in \mathcal{I}$.

Logo,

$$
\left|\bar{A}_{j k} e^{-\sqrt{j^{2}+k^{2}} z} \cos (j x) \cos (k y)\right| \leq \frac{2}{\pi^{2}}\left|<u, \varphi_{j k}>\right|^{2}+\frac{C}{2\left(j^{2}+k^{2}\right)^{2}},
$$

para cada $(x, y, z) \in \mathbb{R}_{+}^{3}$ e para qualquer $(j, k) \in \mathcal{I}$.

Usando a Identidade de Parseval, temos que as séries numéricas

$$
\sum_{(j, k) \in \mathcal{I}}^{\infty} \frac{1}{\left(j^{2}+k^{2}\right)^{2}} \quad \text { e } \quad \sum_{(j, k) \in \mathcal{I}}^{\infty}\left|<u, \varphi_{j k}>\right|^{2},
$$

são convergentes, logo a série

$$
\sum_{(j, k) \in \mathcal{I}}^{\infty} \bar{A}_{j k} e^{-\sqrt{j^{2}+k^{2}} z} \cos (j x) \cos (k y),
$$

define uma função contínua em $[0, \pi] \times[0, \pi] \times(0, \infty)$. Portanto, a função $\tilde{v}$ é contínua em $[0, \pi] \times[0, \pi] \times(0, \infty)$ e assim pela Proposição 14 ., segue que $\tilde{v} \in C_{\text {per }}\left(\mathbb{R}_{+}^{3}\right)$.

2. $\tilde{v} \in C_{\text {per }}^{2}\left(\mathbb{R}_{+}^{3}\right)$.

De fato, derivando em relação a $x$, obtemos séries com termos gerais satisfazendo

$$
\left|j^{m} \bar{A}_{j k} e^{-\sqrt{j^{2}+k^{2}} z} \psi_{j}(x) \cos (k y)\right| \leq \frac{\left|\bar{A}_{j k}\right|^{2}}{2}+\frac{j^{2 m}}{2} e^{-2 \sqrt{j^{2}+k^{2}} z},
$$

onde $\psi_{j}(x)=\cos (j x)$ ou $\psi_{j}(x)=\sin (j x)$, para qualquer $(j, k) \in \mathcal{I}$ e $m \in \mathbb{Z}_{+}^{*}$.

Mas,

$$
j^{2 m} e^{-2 \sqrt{j^{2}+k^{2}} z} \leq j^{2 m} e^{-j z} e^{-\sqrt{j^{2}+k^{2}} z} \leq \frac{C}{\left(j^{2}+k^{2}\right)^{2}}
$$


para qualquer $(j, k) \in \mathcal{I}, m \in \mathbb{Z}_{+}^{*}$ e $z>0$, onde $C$ é constante que depende de $z$.

Logo,

$$
\left|j^{m} \bar{A}_{j k} e^{-\sqrt{j^{2}+k^{2}} z} \psi_{j}(x) \cos (k y)\right| \leq \frac{\left|\bar{A}_{j k}\right|^{2}}{2}+\frac{C}{2\left(j^{2}+k^{2}\right)^{2}},
$$

onde pelos mesmos argumentos usados no item anterior, temos que a série

$$
\sum_{(j, k) \in \mathcal{I}}^{\infty} j^{m} \bar{A}_{j k} e^{-\sqrt{j^{2}+k^{2}} z} \psi_{j}(x) \cos (k y)
$$

define uma função contínua em $[0, \pi] \times[0, \pi] \times(0, \infty)$, ou seja, as derivadas em relação a variável $x$ da função $\tilde{v}$ são contínuas em $[0, \pi] \times[0, \pi] \times(0, \infty)$. Analogamente, temos que as derivadas de $\tilde{v}$ em relação a $y$, bem como suas derivadas mistas, também são contínuas em $[0, \pi] \times[0, \pi] \times(0, \infty)$.

Pela paridade e periodicidade de $\tilde{v}$ na variável $x$, temos que

$$
\frac{\partial \tilde{v}}{\partial x}(0, y, z)=\frac{\partial \tilde{v}}{\partial x}(\pi, y, z)=0
$$

para qualquer $y \in \mathbb{R}, z>0$. Logo, como $\frac{\partial \tilde{v}}{\partial x}(\cdot, y, z)$ é uma extensão ímpar de $\left.\frac{\partial \tilde{v}}{\partial x}(\cdot, y, z)\right|_{[0, \pi]}$ ' segue da Proposição 13., que $\frac{\partial \tilde{v}}{\partial x}(\cdot, y, z) \in C_{\text {per }}(\mathbb{R})$, para qualquer $y \in \mathbb{R}, z>0$.

Por outro lado, $\frac{\partial \tilde{v}}{\partial x}(x, \cdot, z)$ é uma extensão par de $\left.\frac{\partial \tilde{v}}{\partial x}(x, \cdot, z)\right|_{[0, \pi]}$ para qualquer $x \in \mathbb{R}, z>0$. Então, da Proposição 14., temos que $\frac{\partial \tilde{v}}{\partial x}(x, \cdot, z) \in C_{p e r}(\mathbb{R})$, para qualquer $x \in \mathbb{R}, z>0$. Com isso, obtemos que $\frac{\partial \tilde{v}}{\partial x} \in C_{\text {per }}\left(\mathbb{R}_{+}^{3}\right)$. Fazendo a mesma análise, verificamos que $\frac{\partial \tilde{v}}{\partial y} \in C_{p e r}\left(\mathbb{R}_{+}^{3}\right)$.

Analogamente, sabendo que as segundas derivadas da função $\tilde{v}$ em relação as variáveis $x$ e $y$ são funções pares, temos que

$$
\frac{\partial^{2} \tilde{v}}{\partial x^{2}}, \frac{\partial^{2} \tilde{v}}{\partial y^{2}} \in C_{p e r}\left(\mathbb{R}_{+}^{3}\right)
$$

Temos também, que a função $\frac{\partial^{2} \tilde{v}}{\partial x \partial y}$ é ímpar nas variáveis $x$ e $y$, e também temos que

$$
\frac{\partial^{2} \tilde{v}}{\partial x \partial y}(0, y, z)=\frac{\partial^{2} \tilde{v}}{\partial x \partial y}(\pi, y, z)=0
$$

para qualquer $y \in \mathbb{R}, z>0 \mathrm{e}$

$$
\frac{\partial^{2} \tilde{v}}{\partial x \partial y}(x, 0, z)=\frac{\partial^{2} \tilde{v}}{\partial x \partial y}(x, \pi, z)=0
$$

para qualquer $x \in \mathbb{R}, z>0$. Então, pela Proposição 13. temos que $\frac{\partial^{2} \tilde{v}}{\partial x \partial y} \in C_{p e r}\left(\mathbb{R}_{+}^{3}\right)$. 
Derivando em relção a $z$, obtemos a série

$$
-\sum_{(j, k) \in \mathcal{I}}^{\infty} \bar{A}_{j k}\left(j^{2}+k^{2}\right)^{\frac{1}{2}} e^{-\sqrt{j^{2}+k^{2}} z} \cos (j x) \cos (k y)
$$

Sabendo que $\left(j^{2}+k^{2}\right)^{\frac{1}{2}} \leq j^{2}+k^{2}$, segue que

$$
\begin{aligned}
\left|\bar{A}_{j k}\left(j^{2}+k^{2}\right)^{\frac{1}{2}} e^{-\sqrt{j^{2}+k^{2}} z} \cos (j x) \cos (k y)\right| & \leq \frac{\left|\bar{A}_{j k}\right|^{2}}{2}+\frac{\left(j^{2}+k^{2}\right)}{2} e^{-2 \sqrt{j^{2}+k^{2}} z} \\
& \leq \frac{\left|\bar{A}_{j k}\right|^{2}}{2}+\frac{M}{2\left(j^{2}+k^{2}\right)^{2}}
\end{aligned}
$$

para cada $(x, y, z) \in \mathbb{R}_{+}^{3}$, onde $M$ é uma constante que depende de $z$.

Logo, pelos mesmos argumentos usados anteriormente segue que a série

$$
\sum_{(j, k) \in \mathcal{I}}^{\infty} \bar{A}_{j k}\left(j^{2}+k^{2}\right)^{\frac{1}{2}} e^{-\sqrt{j^{2}+k^{2}} z} \cos (j x) \cos (k y)
$$

define uma função contínua em $[0, \pi] \times[0, \pi] \times(0, \infty)$, ou seja, a função $\frac{\partial \tilde{v}}{\partial z}$ é continua em $[0, \pi] \times[0, \pi] \times(0, \infty)$ e novamente pela Proposição 14 . temos que $\frac{\partial \tilde{v}}{\partial z} \in C_{p e r}\left(\mathbb{R}_{+}^{3}\right)$.

Analogamente, a série obtida pela segunda derivada em relação a $z$ define uma função contínua em $[0, \pi] \times[0, \pi] \times(0, \infty)$, e assim temos que $\frac{\partial^{2} \tilde{v}}{\partial z^{2}} \in C_{\text {per }}\left(\mathbb{R}_{+}^{3}\right)$. Portanto, $\tilde{v} \in C_{\text {per }}^{2}\left(\mathbb{R}_{+}^{3}\right)$.

3. $\Delta \tilde{v}(x, y, z)=0 \mathrm{em} \mathbb{R}_{+}^{3}$.

De fato, como vimos as séries das derivadas segundas de $\tilde{v}$ convergem uniformemente em compactos, $\log \mathrm{O}$

$$
\begin{aligned}
\Delta \tilde{v}(x, y, x)= & -\sum_{(j, k) \in \mathcal{I}}^{\infty} j^{2} \bar{A}_{j k} e^{-\sqrt{j^{2}+k^{2}} z} \cos (j x) \cos (k y) \\
& -\sum_{(j, k) \in \mathcal{I}}^{\infty} k^{2} \bar{A}_{j k} e^{-\sqrt{j^{2}+k^{2}} z} \cos (j x) \cos (k y) \\
& +\sum_{(j, k) \in \mathcal{I}}^{\infty}\left(j^{2}+k^{2}\right) \bar{A}_{j k} e^{-\sqrt{j^{2}+k^{2}} z} \cos (j x) \cos (k y) \\
= & 0,
\end{aligned}
$$

para qualquer $(x, y, z) \in \mathbb{R}_{+}^{3}$.

4. $\tilde{v}(\cdot, \cdot, 0)=\tilde{u}(\cdot, \cdot)$ quase sempre em $\mathbb{R}^{2}$.

De fato, dada $u \in D\left(A_{\frac{1}{2}}\right) \subset X$, temos que

$$
u=\sum_{(j, k) \in \mathcal{I}}^{\infty}<u, \varphi_{j k}>\varphi_{j k}
$$


em $L^{2}\left(\Omega_{q}\right)$, onde $\varphi_{j k}$ são as autofunções de $-\Delta_{N}$ em $\Omega_{q}$. Em particular

$$
\tilde{u}=\sum_{(j, k) \in \mathcal{I}}^{\infty}<u, \varphi_{j k}>\varphi_{j k}
$$

em $L_{p e r}^{2}\left(\mathbb{R}^{2}\right)$.

Por outro lado, considere a sequência $\left\{\psi_{m n}\right\} \subset C_{\text {per }}^{\infty}\left(\overline{\mathbb{R}_{+}^{3}}\right)$ tal que

$$
\psi_{m n}(x, y, z)=\sum_{(j, k) \in \mathcal{I}}^{m, n} \bar{A}_{j k} e^{-\sqrt{j^{2}+k^{2}} z} \cos (j x) \cos (k y),
$$

e observe que

(a)

$$
\begin{aligned}
\left\|\tilde{v}-\psi_{m n}\right\|_{L_{p e r}^{2}\left(\mathbb{R}_{+}^{3}\right)}^{2} & =\int_{0}^{\infty} \sum_{\substack{(j, k) \in \mathcal{I} \\
j>m \text { ou } k>n}}^{\infty}\left|\bar{A}_{j k}\right|^{2} e^{-2 \sqrt{j^{2}+k^{2}} z} d z \\
& \leq \sum_{\substack{(j, k) \in \mathcal{I} \\
j>m \text { ou } k>n}}^{\infty}\left|\bar{A}_{j k}\right|^{2} \int_{0}^{\infty} e^{-2 z} d z \\
& \leq \sum_{\substack{(j, k) \in \mathcal{I} \\
j>m \text { ou } k>n}}^{\infty} \frac{\left|\bar{A}_{j k}\right|^{2}}{2} \stackrel{m, n \rightarrow \infty}{\longrightarrow} 0 .
\end{aligned}
$$

(b)

$$
\begin{aligned}
\left\|\nabla \tilde{v}-\nabla \psi_{m n}\right\|_{L_{p e r}^{2}\left(\mathbb{R}_{+}^{3}\right)}^{2} \leq & \sum_{\substack{(j, k) \in \mathcal{I} \\
j>m \text { ou } k>n}}^{\infty} \frac{j^{2}\left|\bar{A}_{j k}\right|^{2}}{2} \\
& +\sum_{\substack{(j, k) \in \mathcal{I} \\
j>m \text { ou } k>n}}^{\infty} \frac{k^{2}\left|\bar{A}_{j k}\right|^{2}}{2} \\
& +\sum_{\substack{(j, k) \in \mathcal{I} \\
j>m \text { ou } k>n}}^{\infty} \frac{\left(j^{2}+k^{2}\right)\left|\bar{A}_{j k}\right|^{2}}{2} \stackrel{m, n \rightarrow \infty}{\longrightarrow 0} .
\end{aligned}
$$

Então, concluímos que $\tilde{v} \in H_{\text {per }}^{1}\left(\mathbb{R}_{+}^{3}\right)$, pois

$$
\lim _{m, n \rightarrow \infty}\left\|\tilde{v}-\psi_{m n}\right\|_{H_{p e r}^{1}\left(\mathbb{R}_{+}^{3}\right)}=0 .
$$


Pelo Teorema do Traço, temos que

$$
\begin{aligned}
\tilde{v}(\cdot, \cdot, 0) & =\sum_{(j, k) \in \mathcal{I}}^{\infty} \bar{A}_{j k} \cos (j x) \cos (k y) \\
& =\sum_{(j, k) \in \mathcal{I}}^{\infty}<u, \varphi_{j k}>\varphi_{j k} .
\end{aligned}
$$

Portanto, $\tilde{v}(\cdot, \cdot, 0)=\tilde{u}(\cdot, \cdot)$ quase sempre em $\mathbb{R}^{2}$.

5. $\lim _{z \rightarrow \infty}\|\tilde{v}(\cdot, \cdot, z)\|_{L^{2}\left(\Omega_{q}\right)}=0$.

De fato, observe que

$$
\begin{aligned}
\int_{\Omega_{q}}|\tilde{v}(x, y, z)|^{2} d x d y & =\int_{\Omega_{q}}\left|\sum_{(j, k) \in \mathcal{I}}^{\infty} \bar{A}_{j k} e^{-\sqrt{j^{2}+k^{2}} z} \cos (j x) \cos (k y)\right|^{2} d x d y \\
& =\sum_{(j, k) \in \mathcal{I}}^{\infty}\left|\bar{A}_{j k}\right|^{2} e^{-2 \sqrt{j^{2}+k^{2}} z} \\
& \leq\left(\sum_{(j, k) \in \mathcal{I}}^{\infty}\left|\bar{A}_{j k}\right|^{2}\right) e^{-2 z}
\end{aligned}
$$

para qualquer $z>0$. Então, pela convergência da série numérica da desigualdade anterior, temos que

$$
\lim _{z \rightarrow \infty} \int_{\Omega_{q}}|\tilde{v}(x, y, z)|^{2} d x d y=0 .
$$

Portanto, $\lim _{z \rightarrow \infty}\|\tilde{v}(\cdot, \cdot, z)\|_{L^{2}\left(\Omega_{q}\right)}=0$.

6. $\lim _{z \rightarrow \infty}\left\|\tilde{v}_{z}(\cdot, \cdot, z)\right\|_{L^{2}\left(\Omega_{q}\right)}=0$.

De fato, este item é análogo ao item anterior.

7.

$$
\int_{\Omega_{q}} \tilde{v}(x, y, z) d x d y=0
$$

para qualquer $z \geq 0$.

De fato, usando a convergência uniforme de séries de funções, temos que

$$
\int_{\Omega_{q}} \tilde{v}(x, y, z) d x d y=\sum_{(j, k) \in \mathcal{I}}^{\infty} \bar{A}_{j k} e^{-\sqrt{j^{2}+k^{2}} z}\left(\int_{\Omega_{q}} \cos (j x) \cos (k y) d x d y\right)=0
$$

para qualquer $z>0$. 
Para o caso $z=0$, observe que

$$
\begin{aligned}
\left|\int_{\Omega_{q}} \tilde{v}(x, y, 0) d x d y\right| & =\left|\int_{\Omega_{q}} \tilde{v}(x, y, 0)-\tilde{u}(x, y) d x d y\right| \\
& \leq \int_{\Omega_{q}}|\tilde{v}(x, y, 0)-\tilde{u}(x, y)| d x d y \\
& =0,
\end{aligned}
$$

pois $\tilde{v}(\cdot, \cdot, 0)=\tilde{u}(\cdot, \cdot)$ quase sempre em $\mathbb{R}^{2}$.

Portanto,

$$
\int_{\Omega_{q}} \tilde{v}(x, y, z) d x d y=0
$$

para qualquer $z \geq 0$.

Portanto, concluímos finalmente que $\tilde{v}$ é de fato solução clássica de (4.1) e a unicidade de solução segue analogamente ao caso do domínio $\Omega_{i}$.

Com isso, temos a seguinte definição.

Definição 12. Seja

$$
D\left(A_{\frac{1}{2}}\right)=\left\{u \in H^{s}\left(\Omega_{q}\right)\left|s>2, \quad \frac{\partial u}{\partial n}\right|_{\partial \Omega_{q}}=0 e \int_{\Omega_{q}} u(x, y) d x d y=0\right\} .
$$

Definimos o seguinte operador

$$
\begin{aligned}
A_{\frac{1}{2}}: D\left(A_{\frac{1}{2}}\right) \subset X & \longrightarrow X \\
u & \longmapsto-\left.\left(\tilde{v}_{z}(\cdot, \cdot,, 0)\right)\right|_{\Omega_{q}},
\end{aligned}
$$

onde ṽ é a única solução clássica (4.1), para o caso $\Omega_{q}$.

Observação 4. Analogamente ao caso do domínio $\Omega_{i}$ temos que o operador $A_{\frac{1}{2}}$ está bem definido $e$

$$
A_{\frac{1}{2}} u=\sum_{(j, k) \in \mathcal{I}}^{\infty} \lambda_{j k}^{\frac{1}{2}}<u, \varphi_{j k}>\varphi_{j k}
$$

onde $\varphi_{j k}$ e $\lambda_{j k}$ são, respectivamente, as autofunções e os autovalores do operador $-\Delta_{N}$ em $\Omega_{q}$.

Tendo definido o operador $A_{\frac{1}{2}}$ podemos definir o operador $B_{\frac{1}{2}}$.

Definição 13. Seja

$$
Y=\left\{u \in X\left|\sum_{(j, k) \in \mathcal{I}}^{\infty} \lambda_{j k}\right|<u, \varphi_{j k}>\left.\right|^{2}<\infty\right\} .
$$

Definimos o operador

$$
\begin{aligned}
B_{\frac{1}{2}}: Y \subset X & \longrightarrow X \\
u & \longmapsto B_{\frac{1}{2}} u=\sum_{(j, k) \in \mathcal{I}}^{\infty} \lambda_{j k}^{\frac{1}{2}}<u, \varphi_{j k}>\varphi_{j k}
\end{aligned}
$$


onde $\lambda_{j k}$ e $\varphi_{j k}$ são, respectivamente, os autovalores e autofunções do operador $-\Delta_{N}$, para qualquer $(j, k) \in \mathcal{I}$.

Observe que a convergência acima é na norma de $X$, isto é,

$$
\lim _{m, n \rightarrow \infty}\left\|B_{\frac{1}{2}} u-\sum_{(j, k) \in \mathcal{I}}^{m, n} \lambda_{j k}^{\frac{1}{2}}<u, \varphi_{j k}>\varphi_{j k}\right\|=0 .
$$

Lema 8. O operador $B_{\frac{1}{2}}$ está bem definido e é uma extensão do operador $A_{\frac{1}{2}}$ a $Z$.

Demonstração. Seja $u \in Y$, logo a série

$$
\sum_{(j, k) \in \mathcal{I}}^{\infty} \lambda_{j k}^{\frac{1}{2}}<u, \varphi_{j k}>\varphi_{j k}
$$

converge em $L^{2}\left(\Omega_{q}\right)$, ou seja, $B_{\frac{1}{2}} u \in L^{2}\left(\Omega_{q}\right)$.

Por outro lado, como

$$
\int_{\Omega_{q}} \varphi_{j k}(x, y) d x d y=0
$$

para qualquer $(j, k) \in \mathcal{I}$, segue que

$$
\begin{aligned}
\left|\int_{\Omega_{q}} B_{\frac{1}{2}} u(x, y) d x d y\right| & \leq \int_{\Omega_{q}}\left|B_{\frac{1}{2}} u(x, y)-\sum_{(j, k) \in \mathcal{I}}^{m, n} \lambda_{j k}^{\frac{1}{2}}<u, \varphi_{j k}>\varphi_{j k}(x, y)\right| d x d y \\
& \leq \pi\left\|B_{\frac{1}{2}} u-\sum_{(j, k) \in \mathcal{I}}^{m, n} \lambda_{j k}^{\frac{1}{2}}<u, \varphi_{j k}>\varphi_{j k}\right\| \stackrel{m, n \rightarrow \infty}{\longrightarrow 0},
\end{aligned}
$$

ou seja,

$$
\int_{\Omega_{q}} B_{\frac{1}{2}} u(x, y) d x d y=0
$$

e assim $B_{\frac{1}{2}} u \in X$. Então, devido a unicidade da Série de Fourier, o operador está bem definido.

Para verificar que o operador $B_{\frac{1}{2}}$ é uma extensão do operador $A_{\frac{1}{2}}$, precisamos somente mostrar que $B_{\frac{1}{2}}$ coincide com $A_{\frac{1}{2}}$ sobre o $D\left(A_{\frac{1}{2}}\right)$, pois analogamente ao caso do domínio $\Omega_{i}$ temos que $D\left(A_{\frac{1}{2}}\right) \subset Y$.

Seja $u \in D\left(A_{\frac{1}{2}}\right)$.

$$
\begin{aligned}
\left\|A_{\frac{1}{2}} u-B_{\frac{1}{2}} u\right\|_{L^{1}\left(\Omega_{q}\right)} & \leq \int_{\Omega_{q}}\left|A_{\frac{1}{2}} u(x, y)-\sum_{(j, k) \in \mathcal{I}}^{m, n} \lambda_{j k}^{\frac{1}{2}}<u, \varphi_{j k}>\varphi_{j k}(x, y)\right| d x d y \\
& +\int_{\Omega_{q}}\left|\sum_{(j, k) \in \mathcal{I}}^{m, n} \lambda_{j k}^{\frac{1}{2}}<u, \varphi_{j k}>\varphi_{j k}(x, y)-B_{\frac{1}{2}} u(x, y)\right| d x d y \\
& \leq \pi\left\|A_{\frac{1}{2}} u-\sum_{(j, k) \in \mathcal{I}}^{m, n} \lambda_{j k}^{\frac{1}{2}}<u, \varphi_{j k}>\varphi_{j k}\right\| \\
& +\pi\left\|B_{\frac{1}{2}} u-\sum_{(j, k) \in \mathcal{I}}^{m, n} \lambda_{j k}^{\frac{1}{2}}<u, \varphi_{j k}>\varphi_{j k}\right\| .
\end{aligned}
$$


Porém, sabemos que:

1. Por definição do operador $B_{\frac{1}{2}}$, temos

$$
\left\|B_{\frac{1}{2}} u-\sum_{(j, k) \in \mathcal{I}}^{m, n} \lambda_{j k}^{\frac{1}{2}}<u, \varphi_{j k}>\varphi_{j k}\right\| \stackrel{m, n \rightarrow \infty}{\longrightarrow} 0 .
$$

2. Usando a ortonormalidade das funções $\varphi_{j k}$, para qualquer $(j, k) \in \mathcal{I}$, temos

$$
\begin{aligned}
\left\|A_{\frac{1}{2}} u-\sum_{(j, k) \in \mathcal{I}}^{m, n} \lambda_{j k}^{\frac{1}{2}}<u, \varphi_{j k}>\varphi_{j k}\right\|^{2} & =\left\|A_{\frac{1}{2}} u-\sum_{(j, k) \in \mathcal{I}}^{m, n}<A_{\frac{1}{2}} u, \varphi_{j k}>\varphi_{j k}\right\|^{2} \\
& =\sum_{(j, k) \in \mathcal{I}}^{\infty}\left|<A_{\frac{1}{2}} u, \varphi_{j k}>\right|^{2}-\sum_{(j, k) \in \mathcal{I}}^{m, n}\left|<A_{\frac{1}{2}} u, \varphi_{j k}>\right|^{2},
\end{aligned}
$$

para qualquer $m, n \in \mathbb{N}^{*}, \log 0$

$$
\left\|A_{\frac{1}{2}} u-\sum_{(j, k) \in \mathcal{I}}^{m, n} \lambda_{j k}^{\frac{1}{2}}<u, \varphi_{j k}>\varphi_{j k}\right\| \stackrel{m, n \rightarrow \infty}{\longrightarrow} 0 .
$$

Portanto,

$$
\left\|A_{\frac{1}{2}} u-B_{\frac{1}{2}} u\right\|_{L^{1}\left(\Omega_{q}\right)}=0 \Longrightarrow A_{\frac{1}{2}} u=B_{\frac{1}{2}} u
$$

quase sempre em $\Omega_{q}$, para qualquer $u \in D\left(A_{\frac{1}{2}}\right)$, e assim $B_{\frac{1}{2}}$ é uma extensão de $A_{\frac{1}{2}}$ a $Y$.

Finalmente, temos o seguinte teorema.

Teorema 9. O operador $B_{\frac{1}{2}}$ coincide com o operador $\left(-\Delta_{N}\right)^{\frac{1}{2}}$ em $\Omega_{q}$, ou seja,

$$
<u, B_{\frac{1}{2}} u>\geq 0
$$

para qualquer $u \in D\left(B_{\frac{1}{2}}\right) e$

$$
B_{\frac{1}{2}} \circ B_{\frac{1}{2}} u=-\Delta_{N} u
$$

para qualquer $u \in D\left(-\Delta_{N}\right)$.

Demonstração. A demonstração é análoga ao caso do domínio $\Omega_{i}$.

\subsection{Caso $\Omega_{b}=B(0, \pi)$}

Seja $s>2$, definiremos

$$
D\left(A_{\frac{1}{2}}\right)=\left\{u \in H^{s}(\Omega)\left|\frac{\partial u}{\partial n}\right|_{\partial \Omega_{b}}=0 \text { e } \int_{\Omega_{b}} u(x, y) d x d y=0\right\}
$$

Analogamente, à seção anterior, temos que a derivada de $u$ tem traço bem definido e com isso $D\left(A_{\frac{1}{2}}\right)$ está bem definido.

Considere $u \in D\left(A_{\frac{1}{2}}\right)$, vamos inicialmente estender $u$ à $\mathbb{R}^{2}$ para, então definirmos o operador $A_{\frac{1}{2}}$ em $\Omega_{b}$, como fizemos nos casos anteriores. 
Sabemos que para qualquer $(x, y) \in \Omega_{b}$, vale que $(x, y)=(\alpha \cos \theta, \alpha \sin \theta)$, onde $\alpha \in(-\pi, \pi)$ e $\theta \in \mathbb{R}$.

Sendo assim, para qualquer $(x, y) \in \Omega_{b}$ considere a função $\bar{u}: B(0,3 \pi) \longrightarrow \mathbb{R}$ tal que

$$
\bar{u}(x, y)=\bar{u}(\alpha \cos \theta, \alpha \sin \theta)=\left\{\begin{array}{ll}
u(\alpha \cos \theta, \alpha \sin \theta) & \text { se }-\pi \leq \alpha \leq \pi \\
u((-\alpha-2 \pi) \cos \theta,(-\alpha-2 \pi) \sin \theta) & \text { se }-3 \pi \leq \alpha \leq-\pi
\end{array} .\right.
$$

Observação 5. 1. Se $-3 \pi \leq \alpha \leq-\pi$, então $-\pi \leq-\alpha-2 \pi \leq \pi$, logo

$$
u((-\alpha-2 \pi) \cos \theta,(-\alpha-2 \pi) \sin \theta)
$$

está bem definida.

2. Seja $\left(x_{0}, y_{0}\right) \in B(0,3 \pi)$, tal que $\left(x_{0}, y_{0}\right)=(\beta \cos \theta, \beta \sin \theta)$ onde $\beta=-\pi-s$, com $s \in[0,2 \pi]$.

Logo, como $-3 \pi \leq \beta \leq-\pi$, temos por definição que

$$
\begin{aligned}
\bar{u}(\beta \cos \theta, \beta \sin \theta) & =u((-\beta-2 \pi) \cos \theta,(-\beta-2 \pi) \sin \theta) \\
& =u((s-\pi) \cos \theta,(s-\pi) \sin \theta) .
\end{aligned}
$$

Por outro lado, considere o ponto $\left(x_{1}, y_{1}\right)=(\alpha \cos \bar{\theta}, \alpha \sin \bar{\theta})$, onde $\bar{\theta}=\theta+\pi$ e $\alpha=-3 \pi+s$. Para que $\bar{u}$ esteja bem definida na $B(0,3 \pi)$, precisamos verificar se $\bar{u}\left(x_{0}, y_{0}\right)=\bar{u}\left(x_{1}, y_{1}\right)$. Mas isto ocorre, pois por definição, temos que

$$
\begin{aligned}
\bar{u}(\alpha \cos (\theta+\pi), \alpha \sin (\theta+\pi)) & =u((-\alpha-2 \pi) \cos (\theta+\pi),(-\alpha-2 \pi) \sin (\theta+\pi)) \\
& =u((\pi-s) \cos (\theta+\pi),(\pi-s) \sin (\theta+\pi)) \\
& =u((s-\pi) \cos \theta,(s-\pi) \sin \theta) .
\end{aligned}
$$

Então,

$$
\bar{u}(\beta \cos \theta, \beta \sin \theta)=\bar{u}(\alpha \cos \bar{\theta}, \alpha \sin \bar{\theta}),
$$

para qualquer $\theta \in \mathbb{R}$, onde $\bar{\theta}=\theta+\pi, \beta=-\pi-s$ e $\alpha=-3 \pi+s$, com $s \in[0,2 \pi]$.

Portanto, a função $\bar{u}$ calculada nos pontos com $\alpha \in[\pi, 3 \pi]$ resulta no mesmo valor da função $\bar{u}$ calculada nos pontos com $\alpha \in[-3 \pi,-\pi]$ e assim $\bar{u}$ está bem denifida na $B(0,3 \pi)$.

3. Para facilitar, usaremos letras maiúsculas para denotar uma função nas coordenadas $\alpha$ e $\theta$, como por exemplo, $U(\alpha, \theta)=u(\alpha \cos \theta, \alpha \sin \theta)$.

Considere a extensão radial de $\bar{u}$ com período $4 \pi$, isto é, $\tilde{u}: \mathbb{R}^{2} \longrightarrow \mathbb{R}$ tal que

$$
\begin{aligned}
\tilde{u}(x, y) & =\tilde{U}(\alpha, \theta) \\
& =\left\{\begin{array}{ll}
U(\alpha-4 k \pi, \theta) & \text { se } \quad(4 k-1) \pi \leq \alpha \leq(4 k+1) \pi \\
U(-\alpha+2(2 k-1) \pi, \theta) & \text { se } \quad(4 k-3) \pi \leq \alpha \leq(4 k-1) \pi
\end{array},\right.
\end{aligned}
$$

$\operatorname{com} k \in \mathbb{Z}$. 
Lema 9. A função definida em (4.7) satisfaz as seguintes propriedades

1. $\tilde{U}(\alpha+4 k \pi, \theta)=\tilde{U}(\alpha, \theta)$, para qualquer $\alpha, \theta \in \mathbb{R}, k \in \mathbb{Z}$.

2. $\tilde{U}(-\alpha-2 \pi, \theta)=\tilde{U}(\alpha, \theta)$, para qualquer $\alpha, \theta \in \mathbb{R}$.

3. $\tilde{u} \in C\left(\mathbb{R}^{2}\right)$.

Demonstração. Sejam $\alpha, \theta \in \mathbb{R}$ e $k \in \mathbb{Z}$. Vejamos cada caso de $\alpha$.

i)

$$
\begin{aligned}
(4 k-1) \pi \leq \alpha \leq(4 k+1) \pi & \Longrightarrow(4(2 k)-1) \pi \leq \alpha+4 k \pi \leq(4(2 k)+1) \pi \\
& \Longrightarrow \tilde{U}(\alpha+4 k \pi, \theta)=U(\alpha-4 k \pi, \theta) .
\end{aligned}
$$

ii)

$$
\begin{aligned}
(4 k-3) \pi \leq \alpha \leq(4 k-1) \pi & \Longrightarrow(4(2 k)-3) \pi \leq \alpha+4 k \pi \leq(4(2 k)-1) \pi \\
& \Longrightarrow \tilde{U}(\alpha+4 k \pi, \theta)=U(-\alpha+2(2 k-1) \pi, \theta) .
\end{aligned}
$$

Portanto, $\tilde{U}(\alpha+4 k \pi, \theta)=\tilde{U}(\alpha, \theta)$, para qualquer $\alpha, \theta \in \mathbb{R}$ e $k \in \mathbb{Z}$. A demonstração do item 2. é análoga.

Para provarmos o item 3., observamos primeiramente que como $\tilde{u}$ é uma extensão radialmente periódica de $u \in C^{1}\left(\Omega_{b}\right)$, segue que $\tilde{u}$ é contínua em todos os pontos

$$
(x, y)=(\alpha \cos \theta, \alpha \sin \theta)
$$

exceto, possivelmente, nos pontos $\alpha=(2 n+1) \pi$, com $n \in \mathbb{Z}$. Pela periodicidade de $\tilde{u}$, para provarmos a continuidade de $\tilde{u}$ nestes pontos, basta provarmos que $\tilde{u}$ é contínua nos pontos $\alpha=\pi$ e $\alpha=-\pi$.

Mas isto é verdade pois

$$
\begin{aligned}
& \lim _{\alpha \rightarrow \pi^{+}} \tilde{U}(\alpha, \theta)=\lim _{\alpha \rightarrow \pi^{+}} U(-\alpha+2 \pi, \theta)=U(\pi, \theta) \\
& \lim _{\alpha \rightarrow \pi^{-}} \tilde{U}(\alpha, \theta)=\lim _{\alpha \rightarrow \pi^{-}} U(\alpha, \theta) \quad=U(\pi, \theta) .
\end{aligned}
$$

Portanto,

$$
\lim _{\alpha \rightarrow \pi} \tilde{U}(\alpha, \theta)=\tilde{U}(\pi, \theta),
$$

para qualquer $\theta \in \mathbb{R}$, e assim temos que $\tilde{U}$ é contínua em $\alpha=\pi$.

Usando os mesmos argumentos, verificamos facilmente que $\tilde{U}$ é contínua em $\alpha=-\pi$, então $\tilde{u}$ é contínua em $\mathbb{R}^{2}$.

Tendo obtido a extensão de $u$ em $\mathbb{R}^{2}$, nosso objetivo agora, é verificar a existência e unicidade de solução clássica do problema (4.1) em $\Omega_{b}$, onde $\tilde{u}$ é dada por (4.7). Vejamos, então a definição de solução fraca do problema (4.1) para $\Omega_{b}$. 
Definição 14. Dizemos que uma função $\tilde{v}: \mathbb{R}_{+}^{3} \longrightarrow \mathbb{R}$ é solução clássica do problema (4.1) para $\Omega_{b}$, se satisfaz

1. $\tilde{v} \in C^{2}\left(\mathbb{R}_{+}^{3}\right)$.

2. $\tilde{V}(\alpha+4 k \pi, \theta, z)=\tilde{V}(\alpha, \theta, z)$, para qualquer $\alpha, \theta \in \mathbb{R}, z \geq 0, k \in \mathbb{Z}$.

3. $\tilde{V}(-\alpha-2 \pi, \theta, z)=\tilde{V}(\alpha, \theta, z)$, para qualquer $\alpha, \theta \in \mathbb{R}, z \geq 0$.

4. $\tilde{v}$ satisfaz (4.1).

Para encontrarmos uma candidata à solução clássica de (4.1), vamos inicialmente encontrar uma solução clássica para o problema

$$
\left\{\begin{array}{lll}
\Delta v(x, y, z)=0 & \text { em } & \Omega_{b} \times(0, \infty) \\
v(x, y, 0)=u(x, y) & \text { em } & \Omega_{b} \\
\frac{\partial v}{\partial n}(x, y, z)=0 & \text { em } & \partial \Omega_{b} \times[0, \infty) \\
\lim _{z \rightarrow \infty}\|v(\cdot, \cdot, z)\|_{L^{2}\left(\Omega_{b}\right)}=0 & & \\
\lim _{z \rightarrow \infty}\left\|v_{z}(\cdot, \cdot, z)\right\|_{L^{2}\left(\Omega_{b}\right)}=0 & & \\
(v(\cdot, \cdot, z))_{\Omega_{b}}=0 & \forall z \geq 0 &
\end{array}\right.
$$

onde $n$ é o vetor normal, unitário, exterior ao $\partial \Omega_{b}$, depois vamos extendê-la à $\mathbb{R}_{+}^{3}$, de forma que seja radialmente $4 \pi$-periódica e simétrica em relação ao bordo de $\Omega_{b}$, e verificaremos que esta será a solução de (4.1).

Usando separação de variáveis, procuramos soluções da forma $v(x, y, z)=w(x, y) Z(z)$. Logo, de (4.8), obtemos os seguintes problemas

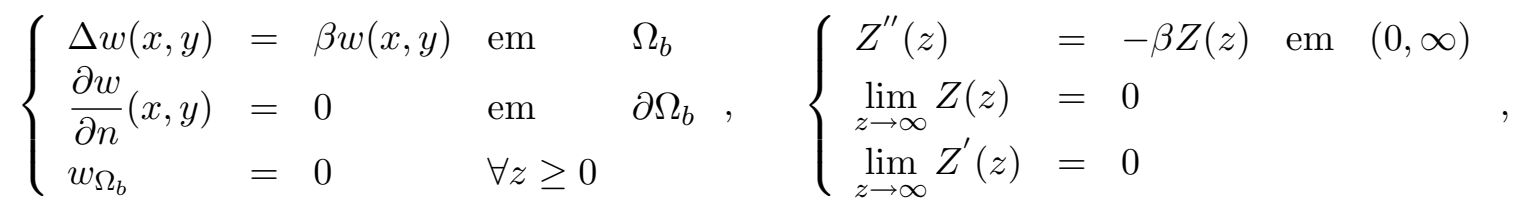

$\operatorname{com} \beta \in \mathbb{R}$.

Consideremos as coordenadas polares

$$
\left\{\begin{array}{l}
x=r \cos \theta \\
y=r \sin \theta
\end{array},\right.
$$

$\operatorname{com} r \in[0, \pi), \theta \in \mathbb{R}$ e $W(r, \theta)=w(r \cos \theta, r \sin \theta)$.

Logo, novamente procurando soluções através do Método de Separação de Variáveis, com

$$
W(r, \theta)=X(r) Y(\theta)
$$


temos os seguinte problemas

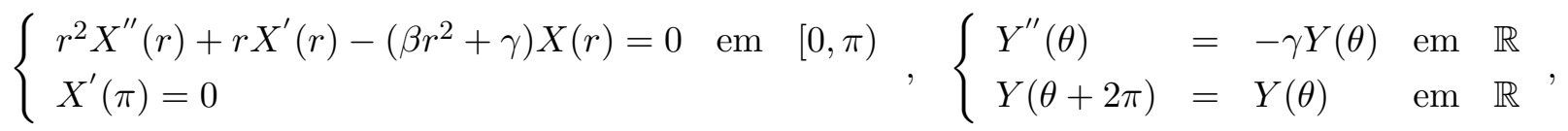

$$
\begin{aligned}
& \begin{cases}Z^{\prime \prime}(z) & =-\beta Z(z) \text { em }(0, \infty) \\
\lim _{z \rightarrow \infty} Z(z) & =0 \\
\lim _{z \rightarrow \infty} Z^{\prime}(z) & =0\end{cases}
\end{aligned}
$$

onde $\beta, \gamma \in \mathbb{R}$.

Através de alguns cálculos, temos que as soluções gerais destes problemas são dadas por

$$
\begin{gathered}
X_{j k}(r)=C_{j k} J_{k}\left(\frac{\mu_{j k}}{\pi} r\right), \quad Y_{k}(\theta)=A_{k} \cos (k \theta)+B_{k} \sin (k \theta) \\
Z_{j k}(z)=E_{j k} e^{-\frac{\mu_{j k}}{\pi} z}
\end{gathered}
$$

onde $\mu_{j k}$ são os zeros positivos de $J_{k}^{\prime}$, para qualquer $j \geq 1, k \geq 0$.

Usando o fato da solução de (4.8) ter média nula e a série de Fourier-Bessel de $u$, temos que a função

$$
V(r, \theta, z)=\sum_{(j, k) \in \mathcal{I}}^{\infty} e^{-\frac{\mu_{j k}}{\pi} z} J_{k}\left(\frac{\mu_{j k}}{\pi} r\right)\left[a_{j k} \cos (k \theta)+b_{j k} \sin (k \theta)\right],
$$

é uma candidata à solução do problema (4.8), onde

$$
\begin{aligned}
a_{j k} & =\frac{2 \mu_{j k}^{2}}{\pi^{3}\left(\mu_{j k}^{2}-k^{2}\right) J_{k}^{2}\left(\mu_{j k}\right)} \int_{0}^{2 \pi} \int_{0}^{\pi} r U(r, \theta) \cos (k \theta) J_{k}\left(\frac{\mu_{j k}}{\pi} r\right) d r d \theta, \\
b_{j k} & =\frac{2 \mu_{j k}^{2}}{\pi^{3}\left(\mu_{j k}^{2}-k^{2}\right) J_{k}^{2}\left(\mu_{j k}\right)} \int_{0}^{2 \pi} \int_{0}^{\pi} r U(r, \theta) \sin (k \theta) J_{k}\left(\frac{\mu_{j k}}{\pi} r\right) d r d \theta,
\end{aligned}
$$

para qualquer $(j, k) \in \mathcal{I}$.

Proposição 10. A função

$$
V(r, \theta, z)=\sum_{(j, k) \in \mathcal{I}}^{\infty} e^{-\frac{\mu_{j k}}{\pi} z} J_{k}\left(\frac{\mu_{j k}}{\pi} r\right)\left[a_{j k} \cos (k \theta)+b_{j k} \sin (k \theta)\right]
$$

onde $a_{j k}, b_{j k}$ são dados, respectivamente, por (4.9) e (4.10), pertence à $C^{2}([0, \pi) \times \mathbb{R} \times(0, \infty))$.

Demonstração. Para verificarmos a suavidade de $V$, vamos utilizar o Critério de Weierstrass para séries indexadas com multi-índices.

1. $V$ é contínua em $[0, \pi) \times \mathbb{R} \times(0, \infty)$. 
De fato, como as funções de Bessel são limitadas, segue que

$$
\left|e^{-\frac{\mu_{j k}}{\pi} z} J_{k}\left(\frac{\mu_{j k}}{\pi} r\right)\left[a_{j k} \cos (k \theta)+b_{j k} \sin (k \theta)\right]\right| \leq M e^{-\frac{\mu_{j k}}{\pi} z} \frac{2 \mu_{j k}^{2}}{\pi^{3}\left(\mu_{j k}^{2}-k^{2}\right) J_{k}^{2}\left(\mu_{j k}\right)},
$$

para qualquer $(j, k) \in \mathcal{I}$, onde $M=2 \sqrt{2} \pi\|U\|_{L^{2}((0, \pi) \times(0,2 \pi) ; r)}$.

Sabemos que

$$
e^{-\frac{\mu_{j k}}{\pi} z} \leq \frac{\bar{M}}{\mu_{j k}^{5}}
$$

para qualquer $(j, k) \in \mathcal{I}$ e para cada $z>0$, onde $\bar{M}$ é uma constante que depende de $z$. Logo,

$$
\left|e^{-\frac{\mu_{j k}}{\pi} z} J_{k}\left(\frac{\mu_{j k}}{\pi} r\right)\left[a_{j k} \cos (k \theta)+b_{j k} \sin (k \theta)\right]\right| \leq \frac{2 M \bar{M}}{\pi^{3} \mu_{j k}^{3}\left(\mu_{j k}^{2}-k^{2}\right) J_{k}^{2}\left(\mu_{j k}\right)},
$$

para cada $(x, y, z) \in \mathbb{R}_{+}^{3}$ e para qualquer $(j, k) \in \mathcal{I}$.

Vejamos que a série numérica

$$
\sum_{(j, k) \in \mathcal{I}}^{\infty} \frac{2 M \bar{M}}{\pi^{3} \mu_{j k}^{3}\left(\mu_{j k}^{2}-k^{2}\right) J_{k}^{2}\left(\mu_{j k}\right)}
$$

é convergente.

Sabemos que

$$
J_{k}^{2}\left(\mu_{j k}\right)=\frac{2}{\mu_{j k}^{2}-k^{2}} \int_{0}^{\mu_{j k}} x J_{k}^{2}(x) d x,
$$

para qualquer $(j, k) \in \mathcal{I}$ e pelo Lema 15 . temos que existe $k_{0}>0$ tal que

$$
J_{k}^{2}(x)>\frac{(e x)^{2 k}}{4 k \pi(2 k)^{2 k}},
$$

para qualquer $k \geq k_{0}$.

Logo,

$$
J_{k}^{2}\left(\mu_{j k}\right) \geq \frac{e^{2 k} \mu_{j k}^{2 k+2}}{4 k \pi(k+1)\left(\mu_{j k}^{2}-k^{2}\right)(2 k)^{2 k}}
$$

então,

$$
\frac{2 M \bar{M}}{\pi^{3} \mu_{j k}^{3}\left(\mu_{j k}^{2}-k^{2}\right) J_{k}^{2}\left(\mu_{j k}\right)}<\frac{16 M \bar{M}}{\pi^{2} \mu_{j k}^{3}} \cdot \frac{k(k+1)(2 k)^{2 k} e^{-2 k}}{2 \mu_{j k}^{2 k+2}}
$$

para qualquer $k \geq k_{0}, j \geq 1$.

Temos ainda que

$$
\frac{k(k+1)(2 k)^{2 k} e^{-2 k}}{2 \mu_{j k}^{2 k+2}} \leq \frac{k(k+1) 2^{2 k} e^{-2 k}}{2 k} \leq 2^{2 k} e^{-2 k} \leq 1,
$$

pois $\mu_{j k}>k$, para qualquer $k>0$. 
Logo,

$$
\frac{2 M \bar{M}}{\pi^{3} \mu_{j k}^{3}\left(\mu_{j k}^{2}-k^{2}\right) J_{k}^{2}\left(\mu_{j k}\right)}<\frac{16 M \bar{M}}{\pi^{2} \mu_{j k}^{3}}
$$

para qualquer $k \geq k_{0}, j \geq 1$.

Por outro lado, usando (4.12), temos que

$$
J_{k}^{2}\left(\mu_{j k}\right) \geq \frac{2}{\mu_{j k}^{2}-k^{2}}\left\|J_{k}\right\|_{L^{2}((0, k) ; x)}^{2}>\frac{2 \widetilde{M}}{\mu_{j k}^{2}-k^{2}}
$$

para qualquer $k \in\left(0, k_{0}\right)$ onde $\widetilde{M}=\min _{k \in\left(0, k_{0}\right)}\left\|J_{k}\right\|_{L^{2}((0, k) ; x)}^{2}$.

Então,

$$
\frac{2 M \bar{M}}{\pi^{3} \mu_{j k}^{3}\left(\mu_{j k}^{2}-k^{2}\right) J_{k}^{2}\left(\mu_{j k}\right)}<\frac{M \bar{M}}{\pi^{3} \widetilde{M} \mu_{j k}^{3}}
$$

para qualquer $k \in\left(0, k_{0}\right)$.

Quando $k=0$, temos que $j \geq 2$, assim por (4.12), segue que

$$
J_{0}^{2}\left(\mu_{j 0}\right) \geq \frac{2}{\mu_{j 0}^{2}}\left\|J_{0}\right\|_{L^{2}\left(\left(0, \mu_{20}\right) ; x\right)}^{2},
$$

então

$$
\frac{2 M \bar{M}}{\pi^{3} \mu_{j k}^{3}\left(\mu_{j k}^{2}-k^{2}\right) J_{k}^{2}\left(\mu_{j k}\right)}<\frac{M \bar{M}}{\pi^{3} M_{1} \mu_{j 0}^{3}}
$$

onde $M_{1}=\left\|J_{0}\right\|_{L^{2}\left(\left(0, \mu_{20}\right) ; x\right)}^{2}$.

Tomando

$$
C=\max \left\{\frac{16 M \bar{M}}{\pi^{2}}, \frac{M \bar{M}}{\pi^{3} \widetilde{M}}, \frac{M \bar{M}}{\pi^{3} M_{1}}\right\}
$$

temos por (4.13), (4.14) e (4.15) que

$$
\frac{2 M \bar{M}}{\pi^{3} \mu_{j k}^{3}\left(\mu_{j k}^{2}-k^{2}\right) J_{k}^{2}\left(\mu_{j k}\right)}<\frac{C}{\mu_{j k}^{3}}
$$

para qualquer $(j, k) \in \mathcal{I}$, onde a constante $C$ depende de $z>0$.

Pelos lemas 16. e 17., temos que

$$
\mu_{j k}>\left(\left(j-\frac{5}{4}\right)^{2} \pi^{2}+k^{2}\right)^{\frac{1}{2}}
$$

e

$$
\left(j-\frac{5}{4}\right)^{2} \pi^{2} \geq j^{2}
$$

para qualquer $j>1$ e $k \geq 0$. Então,

$$
\mu_{j k}>\left(j^{2}+k^{2}\right)^{\frac{1}{2}}
$$

para qualquer $j>1, k \geq 0$. 
Quando $j=1$ temos $k \geq 1$, logo

$$
\mu_{1 k}>k>\frac{1}{\sqrt{2}}\left(1+k^{2}\right)^{\frac{1}{2}} .
$$

Então, para qualquer $(j, k) \in \mathcal{I}$, segue que

$$
\frac{1}{\mu_{j k}^{3}}<\frac{2^{\frac{3}{2}}}{\left(j^{2}+k^{2}\right)^{\frac{3}{2}}} .
$$

Substituindo em (4.16), temos que

$$
\frac{2 M \bar{M}}{\pi^{3} \mu_{j k}^{3}\left(\mu_{j k}^{2}-k^{2}\right) J_{k}^{2}\left(\mu_{j k}\right)}<\frac{2^{\frac{3}{2}} C}{\left(j^{2}+k^{2}\right)^{\frac{3}{2}}},
$$

para qualquer $(j, k) \in \mathcal{I}$.

Pela convergência da série numérica

$$
\sum_{(j, k) \in \mathcal{I}}^{\infty} \frac{2^{\frac{3}{2}} C}{\left(j^{2}+k^{2}\right)^{\frac{3}{2}}}
$$

temos que a série

$$
\sum_{(j, k) \in \mathcal{I}}^{\infty} \frac{2 M \bar{M}}{\pi^{3} \mu_{j k}^{3}\left(\mu_{j k}^{2}-k^{2}\right) J_{k}^{2}\left(\mu_{j k}\right)}
$$

é convergente.

Portanto, a série

$$
\sum_{(j, k) \in \mathcal{I}}^{\infty} e^{-\frac{\mu_{j k}}{\pi} z} J_{k}\left(\frac{\mu_{j k}}{\pi} r\right)\left[a_{j k} \cos (k \theta)+b_{j k} \sin (k \theta)\right],
$$

define uma função contínua $[0, \pi) \times \mathbb{R} \times(0, \infty)$ e assim a função $V$ é contínua em $[0, \pi) \times \mathbb{R} \times(0, \infty)$.

2. $V \in C^{2}([0, \pi) \times \mathbb{R} \times(0, \infty))$.

De fato, considere a série cujo termo geral satisfaz

$\left|e^{-\frac{\mu_{j k}}{\pi} z}\left(\frac{\mu_{j k}}{\pi}\right)^{n} J_{k}^{(n)}\left(\frac{\mu_{j k}}{\pi} r\right)\left[a_{j k} \cos (k \theta)+b_{j k} \sin (k \theta)\right]\right| \leq M e^{-\frac{\mu_{j k}}{\pi} z}\left(\frac{\mu_{j k}}{\pi}\right)^{n} \frac{2 \mu_{j k}^{2}}{\pi^{3}\left(\mu_{j k}^{2}-k^{2}\right) J_{k}^{2}\left(\mu_{j k}\right)}$,

para qualquer $(j, k) \in \mathcal{I}$ e $n \in \mathbb{N}$, usando o fato de que as derivadas das funções de Bessel são limitadas.

Sabemos que

$$
e^{-\frac{\mu_{j k}}{\pi} z} \leq \frac{\bar{M}}{\mu_{j k}^{n+5}}
$$

para qualquer $(j, k) \in \mathcal{I}, n \in \mathbb{N}$ e para cada $z>0$, onde $\bar{M}$ é uma constante que depende de $z$. 
Logo,

$$
\left|e^{-\frac{\mu_{j k}}{\pi} z}\left(\frac{\mu_{j k}}{\pi}\right)^{n} J_{k}^{(n)}\left(\frac{\mu_{j k}}{\pi} r\right)\left[a_{j k} \cos (k \theta)+b_{j k} \sin (k \theta)\right]\right| \leq \frac{M \bar{M}}{\pi^{n}} \frac{2}{\pi^{3} \mu_{j k}^{3}\left(\mu_{j k}^{2}-k^{2}\right) J_{k}^{2}\left(\mu_{j k}\right)},
$$

para qualquer $(j, k) \in \mathcal{I}$ e $n \in \mathbb{N}$. Logo, usando os mesmos argumentos vistos anteriormente, temos que a série

$$
\sum_{(j, k) \in \mathcal{I}}^{\infty} e^{-\frac{\mu_{j k}}{\pi} z}\left(\frac{\mu_{j k}}{\pi}\right)^{n} J_{k}^{(n)}\left(\frac{\mu_{j k}}{\pi} r\right)\left[a_{j k} \cos (k \theta)+b_{j k} \sin (k \theta)\right]
$$

define uma função contínua em $[0, \pi) \times \mathbb{R} \times(0, \infty)$, então as derivadas de primeira e segunda ordem da função $V$ em relação as variáveis $z$ e $r$ são contínuas em $[0, \pi) \times \mathbb{R} \times(0, \infty)$.

Para encerrarmos a demonstração deste item, precisamos verificar a continuidade das derivadas da função $V$ em relação a variável $\theta$.

Observe que

$$
\left|J_{k}\left(\frac{\mu_{j k}}{\pi} r\right) e^{\frac{\mu_{j k}}{\pi} z} k^{n}\left[a_{j k} \cos ^{(n)}(k \theta)+b_{j k} \sin ^{(n)}(k \theta)\right]\right| \leq M k^{n} e^{-\frac{\mu_{j k}}{\pi} z} \frac{2 \mu_{j k}^{2}}{\pi^{3}\left(\mu_{j k}^{2}-k^{2}\right) J_{k}^{2}\left(\mu_{j k}\right)},
$$

para qualquer $(j, k) \in \mathcal{I}$ e $n \in \mathbb{N}^{*}$, onde o segundo termo da desigualdade, pode ser limitado pelo termo geral de uma série numérica convergente. Isto pode ser verificado, através dos mesmos argumentos usados para mostrar que a função $V$ é contínua, juntamente com o fato de que

$$
k^{n} e^{-\frac{\mu_{j k}}{\pi} z} \leq k^{n} \frac{\bar{M}}{\mu_{j k}^{5+n}} \leq \frac{\bar{M}}{\mu_{j k}^{5}}
$$

para $n \in \mathbb{N},(j, k) \in \mathcal{I}$ e para cada $z>0$, onde $\bar{M}$ é uma constante que depende de $z$.

Portanto, a função $V \in C^{2}([0, \pi) \times \mathbb{R} \times(0, \infty))$.

Com isso, temos o seguinte teorema.

Teorema 10. Seja $u \in D\left(A_{\frac{1}{2}}\right)$. Então, a função $v: \Omega_{b} \times(0, \infty) \longrightarrow \mathbb{R}$ dada por

$$
v(x, y, z)=V(r, \theta, z)=\sum_{(j, k) \in \mathcal{I}}^{\infty} e^{-\frac{\mu_{j k}}{\pi} z} J_{k}\left(\frac{\mu_{j k}}{\pi} r\right)\left[a_{j k} \cos (k \theta)+b_{j k} \sin (k \theta)\right]
$$

para qualquer $(x, y, z) \in\left(\Omega_{b} \backslash\{(0,0)\}\right) \times(0, \infty)$, onde $a_{j k}$ e $b_{j k}$ são dados por (4.9) e (4.10), respectivamente, $e$

1.

$$
v(0,0, z)=\sum_{j=2}^{\infty} a_{j 0} e^{-\frac{\mu_{j 0}}{\pi} z}
$$


2.

$$
\begin{aligned}
& \frac{\partial v}{\partial x}(0,0, z)=\frac{1}{2 \pi} \sum_{j=1}^{\infty} a_{j 1} \mu_{j 1} e^{-\frac{\mu_{j 1}}{\pi} z} \\
& \frac{\partial v}{\partial y}(0,0, z)=\frac{1}{2 \pi} \sum_{j=1}^{\infty} b_{j 1} \mu_{j 1} e^{-\frac{\mu_{j 1}}{\pi} z} \\
& \frac{\partial v}{\partial z}(0,0, z)=-\frac{1}{\pi} \sum_{j=2}^{\infty} a_{j 0} \mu_{j 0} e^{-\frac{\mu_{j 0}}{\pi} z}
\end{aligned}
$$

3.

$$
\begin{aligned}
\frac{\partial^{2} v}{\partial x^{2}}(0,0, z) & =\frac{1}{4 \pi^{2}} \quad \sum_{j=1}^{\infty} a_{j 2} \mu_{j 2}^{2} e^{-\frac{\mu_{j 2}}{\pi} z}-\frac{1}{2 \pi^{2}} \sum_{j=2}^{\infty} a_{j 0} \mu_{j 0}^{2} e^{-\frac{\mu_{j 0}}{\pi} z} \\
\frac{\partial^{2} v}{\partial y^{2}}(0,0, z) & =-\frac{1}{4 \pi^{2}} \sum_{j=1}^{\infty} a_{j 2} \mu_{j 2}^{2} e^{-\frac{\mu_{j 2}}{\pi} z}-\frac{1}{2 \pi^{2}} \sum_{j=2}^{\infty} a_{j 0} \mu_{j 0}^{2} e^{-\frac{\mu_{j 0}}{\pi} z} \\
\frac{\partial^{2} v}{\partial z^{2}}(0,0, z) & =\frac{1}{\pi^{2}} \sum_{j=2}^{\infty} a_{j 0} \mu_{j 0}^{2} e^{-\frac{\mu_{j 0}}{\pi} z} \\
\frac{\partial^{2} v}{\partial x \partial y}(0,0, z) & =\frac{1}{4 \pi^{2}} \sum_{j=1}^{\infty} b_{j 2} \mu_{j 2}^{2} e^{-\frac{\mu_{j 2}}{\pi} z} \\
\frac{\partial^{2} v}{\partial x \partial z}(0,0, z) & =-\frac{1}{2 \pi^{2}} \sum_{j=1}^{\infty} a_{j 1} \mu_{j 1}^{2} e^{-\frac{\mu_{j 1}}{\pi} z} \\
\frac{\partial^{2} v}{\partial y \partial z}(0,0, z) & =-\frac{1}{2 \pi^{2}} \sum_{j=1}^{\infty} b_{j 1} \mu_{j 1}^{2} e^{-\frac{\mu_{j 1}}{\pi} z}
\end{aligned}
$$

para qualquer $z>0$, é a única solução clássica de (4.8), isto é, $v \in C^{2}\left(\Omega_{b} \times(0, \infty)\right)$ e satisfaz (4.8).

Demonstração. A demonstração será feita por itens.

1. $v \in C^{2}\left(\Omega_{b} \times(0, \infty)\right)$

De fato, como existe uma bijeção entre $\Omega_{b} \backslash\{(0,0)\}$ e $(0, \pi) \times[0,2 \pi)$, e

$$
V \in C^{2}((0, \pi) \times \mathbb{R} \times(0, \infty))
$$

temos que $v \in C^{2}\left(\left(\Omega_{b} \backslash\{(0,0)\}\right) \times(0, \infty)\right)$. Sendo assim, precisamos somente verificar a suavidade de $v$ no ponto $(0,0, z)$, para qualquer $z>0$.

(a) $v$ é contínua em $(0,0, z)$, para qualquer $z>0$.

De fato, seja $\left(x_{m}, y_{m}, z_{m}\right) \stackrel{m \rightarrow \infty}{\longrightarrow}(0,0, z)$, para $z>0$ arbitrário. Vejamos que

$$
\lim _{m \rightarrow \infty} v\left(x_{m}, y_{m}, z_{m}\right)=v(0,0, z)
$$


Observe que, temos uma sequência $\left(r_{m}, \theta_{m}, z_{m}\right)$ associada a sequência $\left(x_{m}, y_{m}, z_{m}\right)$, tal que $r_{m} \stackrel{m \rightarrow \infty}{\longrightarrow} 0 \mathrm{e} z_{m} \stackrel{m \rightarrow \infty}{\longrightarrow} z$.

$$
\begin{aligned}
\left|v\left(x_{m}, y_{m}, z_{m}\right)-v(0,0, z)\right| & =\left|V\left(r_{m}, \theta_{m}, z_{m}\right)-v(0,0, z)\right| \\
& \leq \sum_{j=2}^{\infty}\left|J_{0}\left(\frac{\mu_{j 0}}{\pi} r_{m}\right) e^{-\frac{\mu_{j 0}}{\pi} z_{m}}-e^{-\frac{\mu_{j 0}}{\pi} z}\right|\left|a_{j 0}\right| \\
& +\sum_{j, k \geq 1}^{\infty}\left|J_{k}\left(\frac{\mu_{j k}}{\pi} r_{m}\right)\right| e^{-\frac{\mu_{j k}}{\pi} z_{m}}\left[\left|a_{j k}\right|+\left|b_{j k}\right|\right] .
\end{aligned}
$$

Como

$$
\lim _{m \rightarrow \infty} J_{0}\left(\frac{\mu_{j 0}}{\pi} r_{m}\right)=1 \quad \text { e } \quad \lim _{m \rightarrow \infty} J_{k}\left(\frac{\mu_{j k}}{\pi} r_{m}\right)=0
$$

para qualquer $k \geq 1$, temos que

$$
\lim _{m \rightarrow \infty} v\left(x_{m}, y_{m}, z_{m}\right)=v(0,0, z)
$$

para qualquer $z>0$.

(b) $\frac{\partial v}{\partial x}$ é contínua em $(0,0, z)$, para qualquer $z>0$.

De fato, temos para qualquer $(x, y, z) \in\left(\Omega_{b} \backslash\{(0,0)\}\right) \times(0, \infty)$ que

$$
\frac{\partial v}{\partial x}=\cos \theta \frac{\partial V}{\partial r}-\frac{1}{r} \sin \theta \frac{\partial V}{\partial \theta} .
$$

Logo,

$$
\begin{aligned}
\frac{\partial v}{\partial x}(x, y, z) & =\cos \theta \sum_{\substack{(j, k) \in \mathcal{I} \\
k \neq 1}}^{\infty} \frac{\mu_{j k}}{\pi} J_{k}^{\prime}\left(\frac{\mu_{j k}}{\pi} r\right) e^{-\frac{\mu_{j k}}{\pi} z}\left(a_{j k} \cos (k \theta)+b_{j k} \sin (k \theta)\right) \\
& +\frac{1}{r} \sin \theta \sum_{\substack{j, k \geq 1 \\
k \neq 1}}^{\infty} J_{k}\left(\frac{\mu_{j k}}{\pi} r\right) e^{-\frac{\mu_{j k}}{\pi} z} k\left(-a_{j k} \sin (k \theta)+b_{j k} \cos (k \theta)\right) \\
& +\sum_{j=1}^{\infty}\left[\frac{\mu_{j 1}}{\pi} J_{1}^{\prime}\left(\frac{\mu_{j 1}}{\pi} r\right)-\frac{1}{r} J_{1}\left(\frac{\mu_{j 1}}{\pi} r\right)\right] b_{j 1} e^{-\frac{\mu_{j 1}}{\pi} z} \sin \theta \cos \theta \\
& +\sum_{j=1}^{\infty}\left[\frac{\mu_{j 1}}{\pi} J_{1}^{\prime}\left(\frac{\mu_{j 1}}{\pi} r\right) \cos ^{2} \theta+\frac{1}{r} J_{1}\left(\frac{\mu_{j 1}}{\pi} r\right) \sin ^{2} \theta\right] a_{j 1} e^{-\frac{\mu_{j 1}}{\pi} z}
\end{aligned}
$$

Pelo Lema 14., temos que

$$
\frac{1}{r} J_{1}\left(\frac{\mu_{j 1}}{\pi} r\right)=\frac{\mu_{j 1}}{\pi} J_{1}^{\prime}\left(\frac{\mu_{j 1}}{\pi} r\right)+\frac{\mu_{j 1}}{\pi} J_{2}\left(\frac{\mu_{j 1}}{\pi} r\right)
$$

$\log 0$

$$
\frac{\mu_{j 1}}{\pi} J_{1}^{\prime}\left(\frac{\mu_{j 1}}{\pi} r\right) \cos ^{2}(\theta)+\frac{1}{r} J_{1}\left(\frac{\mu_{j 1}}{\pi} r\right) \sin ^{2} \theta=\frac{\mu_{j 1}}{\pi} J_{1}^{\prime}\left(\frac{\mu_{j 1}}{\pi} r\right)+\frac{\mu_{j 1}}{\pi} J_{2}\left(\frac{\mu_{j 1}}{\pi} r\right) \sin ^{2} \theta .
$$


Sendo assim, segue que

$$
\begin{aligned}
\left|\frac{\partial v}{\partial x}\left(x_{m}, y_{m}, z_{m}\right)-\frac{\partial v}{\partial x}(0,0, z)\right| \leq & \sum_{\substack{(j, k) \in \mathcal{I} \\
k \neq 1}}^{\infty}\left|\frac{\mu_{j k}}{\pi} J_{k}^{\prime}\left(\frac{\mu_{j k}}{\pi} r_{m}\right)\right| e^{-\frac{\mu_{j k}}{\pi} z_{m}}\left(\left|a_{j k}\right|+\left|b_{j k}\right|\right) \\
& +\frac{1}{r_{m}} \sum_{\substack{j, k \geq 1 \\
k \neq 1}}^{\infty}\left|J_{k}\left(\frac{\mu_{j k}}{\pi} r_{m}\right)\right| e^{-\frac{\mu_{j k}}{\pi} z_{m}} k\left(\left|a_{j k}\right|+\left|b_{j k}\right|\right) \\
& +\sum_{j=1}^{\infty}\left|\frac{\mu_{j 1}}{\pi} J_{1}^{\prime}\left(\frac{\mu_{j 1}}{\pi} r_{m}\right)-\frac{1}{r_{m}} J_{1}\left(\frac{\mu_{j 1}}{\pi} r_{m}\right)\right|\left|b_{j 1}\right| e^{-\frac{\mu_{j 1}}{\pi} z_{m}} \\
& +\sum_{j=1}^{\infty} \mid J_{1}^{\prime}\left(\frac{\mu_{j 1}}{\pi} r_{m}\right) e^{-\frac{\mu_{j 1}}{\pi} z_{m}}-\frac{1}{2} e^{-\frac{\mu_{j 1}}{\pi} z\left|\frac{\mu_{j 1}}{\pi}\right| a_{j 1} \mid} \\
& +\sum_{j=1}^{\infty}\left|\frac{\mu_{j 1}}{\pi} J_{2}\left(\frac{\mu_{j 1}}{\pi} r_{m}\right)\right|\left|a_{j 1}\right| e^{-\frac{\mu_{j 1}}{\pi} z_{m}} .
\end{aligned}
$$

Usando a Regra de L'Hospital, temos que

$$
\begin{aligned}
\lim _{m \rightarrow \infty} \frac{\mu_{j 1}}{\pi} J_{1}^{\prime}\left(\frac{\mu_{j 1}}{\pi} r_{m}\right)-\frac{1}{r_{m}} J_{1}\left(\frac{\mu_{j 1}}{\pi} r_{m}\right) & =0 \\
\lim _{m \rightarrow \infty} \frac{1}{r_{m}} J_{k}\left(\frac{\mu_{j k}}{\pi} r_{m}\right) e^{-\frac{\mu_{j k}}{\pi} z_{m}} & =0 \\
\lim _{m \rightarrow \infty} J_{1}^{\prime}\left(\frac{\mu_{j 1}}{\pi} r_{m}\right) e^{-\frac{\mu_{j 1}}{\pi} z_{m}} & =\frac{1}{2} e^{-\frac{\mu_{j 1}}{\pi} z},
\end{aligned}
$$

para qualquer $j \geq 1, k \neq 1$.

E como $J_{k}^{\prime}(0)=0$, para qualquer $k \neq 1$ e $J_{2}(0)=0$, segue que

$$
\lim _{m \rightarrow \infty} \frac{\partial v}{\partial x}\left(x_{m}, y_{m}, z_{m}\right)=\frac{\partial v}{\partial x}(0,0, z) .
$$

Portanto, $\frac{\partial v}{\partial x}$ é contínua em $(0,0, z)$, para qualquer $z>0$.

(c) $\frac{\partial v}{\partial y}$ é contínua em $(0,0, z)$, para qualquer $z>0$.

De fato, temos para qualquer $(x, y, z) \in\left(\Omega_{b} \backslash\{(0,0)\}\right) \times(0, \infty)$, que

$$
\frac{\partial v}{\partial y}(x, y, z)=\sin \theta \frac{\partial V}{\partial r}(r, \theta, z)+\frac{1}{r} \cos \theta \frac{\partial V}{\partial \theta}(r, \theta, z) .
$$


Logo,

$$
\begin{aligned}
\frac{\partial v}{\partial y}(x, y, z) & =\sin \theta \sum_{\substack{(j, k) \in \mathcal{I} \\
k \neq 1}}^{\infty} \frac{\mu_{j k}}{\pi} J_{k}^{\prime}\left(\frac{\mu_{j k}}{\pi} r\right) e^{-\frac{\mu_{j k}}{\pi} z}\left(a_{j k} \cos (k \theta)+b_{j k} \sin (k \theta)\right) \\
& +\frac{1}{r} \cos (\theta) \sum_{\substack{j, k \geq 1 \\
k \neq 1}}^{\infty} J_{k}\left(\frac{\mu_{j k}}{\pi} r\right) e^{-\frac{\mu_{j k}}{\pi} z} k\left(-a_{j k} \sin (k \theta)+b_{j k} \cos (k \theta)\right) \\
& +\sum_{j=1}^{\infty}\left[\frac{\mu_{j 1}}{\pi} J_{1}^{\prime}\left(\frac{\mu_{j 1}}{\pi} r\right)-\frac{1}{r} J_{1}\left(\frac{\mu_{j 1}}{\pi} r\right)\right] a_{j 1} e^{-\frac{\mu_{j 1}}{\pi} z} \sin \theta \cos \theta \\
& +\sum_{j=1}^{\infty}\left[\frac{\mu_{j 1}}{\pi} J_{1}^{\prime}\left(\frac{\mu_{j 1}}{\pi} r\right) \sin ^{2} \theta+\frac{1}{r} J_{1}\left(\frac{\mu_{j 1}}{\pi} r\right) \cos ^{2} \theta\right] b_{j 1} e^{-\frac{\mu_{j 1}}{\pi} z}
\end{aligned}
$$

Analogamente, usando o Lema 14., temos que

$$
\frac{\mu_{j 1}}{\pi} J_{1}^{\prime}\left(\frac{\mu_{j 1}}{\pi} r\right) \sin ^{2} \theta+\frac{1}{r} J_{1}\left(\frac{\mu_{j 1}}{\pi} r\right) \cos ^{2} \theta=\frac{\mu_{j 1}}{\pi} J_{1}^{\prime}\left(\frac{\mu_{j 1}}{\pi} r\right)+\frac{\mu_{j 1}}{\pi} J_{2}\left(\frac{\mu_{j 1}}{\pi} r\right) \cos ^{2} \theta .
$$

Então, segue que

$$
\begin{aligned}
\left|\frac{\partial v}{\partial y}\left(x_{m}, y_{m}, z_{m}\right)-\frac{\partial v}{\partial x}(0,0, z)\right| & \leq \sum_{\substack{(j, k) \in \mathcal{I} \\
k \neq 1}}^{\infty}\left|\frac{\mu_{j k}}{\pi} J_{k}^{\prime}\left(\frac{\mu_{j k}}{\pi} r_{m}\right)\right| e^{-\frac{\mu_{j k}}{\pi} z_{m}}\left(\left|a_{j k}\right|+\left|b_{j k}\right|\right) \\
& +\frac{1}{r_{m}} \sum_{\substack{j, k \geq 1 \\
k \neq 1}}^{\infty}\left|J_{k}\left(\frac{\mu_{j k}}{\pi} r_{m}\right)\right| e^{-\frac{\mu_{j k}}{\pi} z_{m}} k\left(\left|a_{j k}\right|+\left|b_{j k}\right|\right) \\
& +\sum_{j=1}^{\infty}\left|\frac{\mu_{j 1}}{\pi} J_{1}^{\prime}\left(\frac{\mu_{j 1}}{\pi} r_{m}\right)-\frac{1}{r_{m}} J_{1}\left(\frac{\mu_{j 1}}{\pi} r_{m}\right)\right|\left|a_{j 1}\right| e^{-\frac{\mu_{j 1}}{\pi} z_{m}} \\
& +\sum_{j=1}^{\infty}\left|J_{1}^{\prime}\left(\frac{\mu_{j 1}}{\pi} r_{m}\right) e^{-\frac{\mu_{j 1}}{\pi} z_{m}}-\frac{1}{2} e^{-\frac{\mu_{j 1}}{\pi} z \mid}\right| \frac{\mu_{j 1}}{\pi}\left|b_{j 1}\right| \\
& +\sum_{j=1}^{\infty}\left|\frac{\mu_{j 1}}{\pi} J_{2}\left(\frac{\mu_{j 1}}{\pi} r_{m}\right)\right|\left|b_{j 1}\right| e^{-\frac{\mu_{j 1}}{\pi} z_{m}} .
\end{aligned}
$$

Analogamente ao item anterior, pela Regra de L'Hospital, temos que

$$
\lim _{m \rightarrow \infty} \frac{\partial v}{\partial y}\left(x_{m}, y_{m}, z_{m}\right)=\frac{\partial v}{\partial y}(0,0, z)
$$

Portanto, $\frac{\partial v}{\partial y}$ é contínua em $(0,0, z)$, para qualquer $z>0$.

(d) $\frac{\partial v}{\partial z}$ é contínua em $(0,0, z)$, para qualquer $z>0$.

De fato, temos

$$
\left|\frac{\partial v}{\partial z}\left(x_{m}, y_{m}, z_{m}\right)-\frac{\partial v}{\partial z}(0,0, z)\right| \leq \sum_{j=2}^{\infty}\left|J_{0}\left(\frac{\mu_{j 0}}{\pi} r_{m}\right) e^{-\frac{\mu_{j 0}}{\pi} z_{m}}-e^{-\frac{\mu_{j 0}}{\pi} z}\right|\left(\frac{\mu_{j 0}}{\pi}\right)\left|a_{j 0}\right| .
$$


Como,

$$
\lim _{m \rightarrow \infty} J_{0}\left(\frac{\mu_{j 0}}{\pi} r_{m}\right)=1
$$

segue que

$$
\frac{\partial v}{\partial z}\left(x_{m}, y_{m}, z_{m}\right)=\frac{\partial v}{\partial z}(0,0, z)
$$

Portanto, $\frac{\partial v}{\partial z}$ é contínua em $(0,0, z)$, para qualquer $z>0$.

(e) $\frac{\partial^{2} v}{\partial x^{2}}$ é contínua em $(0,0, z)$, para qualquer $z>0$.

De fato, para qualquer $(x, y, z) \in\left(\Omega_{b} \backslash\{(0,0)\}\right) \times(0, \infty)$, temos que

$$
\begin{aligned}
\frac{\partial^{2} v}{\partial x^{2}}(x, y, z) & =\sin ^{2} \theta\left(\frac{1}{r^{2}} \frac{\partial^{2} V}{\partial \theta^{2}}(r, \theta, z)+\frac{1}{r} \frac{\partial V}{\partial r}(r, \theta, z)-\frac{\partial^{2} V}{\partial r^{2}}(r, \theta, z)\right) \\
& +\sin (2 \theta)\left(-\frac{1}{r} \frac{\partial^{2} V}{\partial r \partial \theta}(r, \theta, z)+\frac{1}{r^{2}} \frac{\partial V}{\partial \theta}(r, \theta, z)\right) \\
& +\frac{\partial^{2} V}{\partial r^{2}}(r, \theta, z) .
\end{aligned}
$$

Fazendo algumas contas, vemos que

$$
\left|\frac{\partial^{2} v}{\partial x^{2}}\left(x_{m}, y_{m}, z_{m}\right)-\frac{\partial^{2} v}{\partial x^{2}}(0,0, z)\right| \leq f\left(r_{m}, \theta_{m}, z_{m}\right),
$$

onde $f\left(r_{m}, \theta_{m}, z_{m}\right)$ é dada por

$$
\begin{aligned}
& \sum_{j=1}^{\infty} \mid\left[-\frac{2}{r_{m}^{2}} J_{2}\left(\frac{\mu_{j 2}}{\pi} r_{m}\right)+\left(\frac{\mu_{j 2}}{\pi}\right)^{2} J_{0}\left(\frac{\mu_{j 0}}{\pi} r_{m}\right)-\frac{1}{r_{m}}\left(\frac{\mu_{j 2}}{\pi}\right) J_{2}^{\prime}\left(\frac{\mu_{j 2}}{\pi} r_{m}\right)\right. \\
- & \left.\left(\frac{\mu_{j 2}}{\pi}\right)^{2} J_{2}^{\prime \prime}\left(\frac{\mu_{j 2}}{\pi} r_{m}\right)\right] e^{-\frac{\mu_{j 2}}{\pi} z_{m}} a_{j 2}-\frac{1}{4} a_{j 2}\left(\frac{\mu_{j 2}}{\pi}\right)^{2} e^{-\frac{\mu_{j 2} 2}{\pi} z \mid} \\
+ & \sum_{j=2}^{\infty}\left|\left(\frac{\mu_{j 0}}{\pi}\right)^{2} J_{0}^{\prime \prime}\left(\frac{\mu_{j 0}}{\pi} r_{m}\right) a_{j 0} e^{-\frac{\mu_{j 2}}{\pi} z_{m}}+\frac{1}{2} a_{j 0}\left(\frac{\mu_{j 0}}{\pi}\right)^{2} e^{-\frac{\mu_{j 2}}{\pi} z}\right| \\
+ & \sum_{j=1}^{\infty}\left|-\frac{6}{r_{m}^{2}} J_{2}\left(\frac{\mu_{j 2}}{\pi} r_{m}\right)+\frac{3 \mu_{j 2}}{\pi r_{m}} J_{2}^{\prime}\left(\frac{\mu_{j 2}}{\pi} r_{m}\right)\right| e^{-\frac{\mu_{j 2}}{\pi} z_{m}}\left(\left|a_{j 2}\right|+\left|b_{j 2}\right|\right) \\
+ & \sum_{j=1}^{\infty}\left|\frac{2}{r_{m}^{2}} J_{2}\left(\frac{\mu_{j 2}}{\pi} r_{m}\right)-\frac{2 \mu_{j 2}}{\pi r_{m}} J_{2}^{\prime}\left(\frac{\mu_{j 2}}{\pi} r_{m}\right)+\frac{\mu_{j 2}}{\pi} J_{2}^{\prime \prime}\left(\frac{\mu_{j 2}}{\pi} r_{m}\right)\right| e^{-\frac{\mu_{j 2}}{\pi} z_{m}}\left(\left|a_{j 2}\right|+\left|b_{j 2}\right|\right) \\
+ & \sum_{j=2}^{\infty}\left|\frac{\mu_{j 0}}{\pi r_{m}} J_{0}^{\prime}\left(\frac{\mu_{j 0}}{\pi} r_{m}\right)-\left(\frac{\mu_{j 0}}{\pi}\right)^{2} J_{0}^{\prime \prime}\left(\frac{\mu_{j 0}}{\pi} r_{m}\right)\right| e^{-\frac{\mu_{j 0}}{\pi} z_{m}}\left|a_{j 0}\right| \\
+ & \sum_{\substack{j, k \geq 1 \\
k \neq 2}}^{\infty}\left|-\frac{1}{r_{m}^{2}} J_{k}\left(\frac{\mu_{j k}}{\pi} r_{m}\right) k^{2}+\frac{\mu_{j k}}{\pi r_{m}} J_{k}^{\prime}\left(\frac{\mu_{j k}}{\pi} r_{m}\right)-\left(\frac{\mu_{j k}}{\pi}\right)^{2} J_{k}^{\prime \prime}\left(\frac{\mu_{j k}}{\pi} r_{m}\right)\right| e^{-\frac{\mu_{j k}}{\pi} z_{m}}\left(\left|a_{j k}\right|+\left|b_{j k}\right|\right) \\
+ & \sum_{\substack{j, k \geq 1 \\
k \neq 2}}^{\infty}\left|\left(\frac{\mu_{j k}}{\pi}\right)^{2} J_{k}^{\prime \prime}\left(\frac{\mu_{j k}}{\pi} r_{m}\right)\right| e^{-\frac{\mu_{j k}}{\pi} z_{m}}\left(\left|a_{j k}\right|+\left|b_{j k}\right|\right) \\
+ & \sum_{\substack{j, k \geq 1 \\
k \neq 2}}^{\infty}\left|-\frac{1}{r_{m} \mu_{j k}} J_{k}^{\prime}\left(\frac{\mu_{j k}}{\pi} r_{m}\right)+\frac{1}{r_{m}^{2}} J_{k}\left(\frac{\mu_{j k}}{\pi} r_{m}\right)\right| k e^{-\frac{\mu_{j k}}{\pi} z_{m}}\left(\left|a_{j k}\right|+\left|b_{j k}\right|\right) \mid .
\end{aligned}
$$


Sabendo que

$$
J_{1}^{\prime}(0)=\frac{1}{2}, J_{k}^{\prime}(0)=0
$$

para qualquer $k \neq 1$,

$$
J_{0}^{\prime \prime}(0)=-\frac{1}{2}, J_{2}^{\prime \prime}(0)=\frac{1}{4} \text { e } J_{k}^{\prime \prime}(0)=0,
$$

para qualquer $k \neq 0,2$, e usando a Regra de L'Hospital, temos que

$$
\lim _{m \rightarrow \infty} \frac{\partial^{2} v}{\partial x^{2}}\left(x_{m}, y_{m}, z_{m}\right)=\frac{\partial^{2} v}{\partial x^{2}}(0,0, z)
$$

e portanto, $\frac{\partial^{2} v}{\partial x^{2}}$ é contínua em $(0,0, z)$, para qualquer $z>0$.

(f) $\frac{\partial^{2} v}{\partial y^{2}}$ é contínua em $(0,0, z)$, para qualquer $z>0$.

De fato, primeiramente observe que

$$
\begin{aligned}
\frac{\partial^{2} v}{\partial y^{2}}(x, y, z)= & -\sin ^{2} \theta\left(\frac{1}{r^{2}} \frac{\partial^{2} V}{\partial \theta^{2}}(r, \theta, z)+\frac{1}{r} \frac{\partial V}{\partial r}(r, \theta, z)-\frac{\partial^{2} V}{\partial r^{2}}(r, \theta, z)\right) \\
& -\sin (2 \theta)\left(\frac{1}{r^{2}} \frac{\partial V}{\partial \theta}(r, \theta, z)-\frac{1}{r} \frac{\partial^{2} V}{\partial r \partial \theta}(r, \theta, z)\right) \\
& -\frac{1}{r^{2}} \frac{\partial^{2} V}{\partial \theta^{2}}(r, \theta, z)+\frac{1}{r} \frac{\partial V}{\partial r}(r, \theta, z) \\
= & -\frac{\partial^{2} v}{\partial x^{2}}(x, y, z)+\frac{\partial^{2} V}{\partial r^{2}}(r, \theta, z)+\frac{1}{r^{2}} \frac{\partial^{2} V}{\partial \theta^{2}}(r, \theta, z)+\frac{1}{r} \frac{\partial V}{\partial r}(r, \theta, z),
\end{aligned}
$$

para qualquer $(x, y, z) \in\left(\Omega_{b} \backslash\{(0,0)\}\right) \times(0, \infty)$.

Por outro lado, para qualquer $z>0$, temos que

$$
\frac{\partial^{2} v}{\partial y^{2}}(0,0, z)=-\frac{\partial^{2} v}{\partial x^{2}}(0,0, z)-\sum_{j=2}^{\infty} a_{j 0}\left(\frac{\mu_{j 0}}{\pi}\right)^{2} e^{-\frac{\mu_{j 0}}{\pi} z} .
$$

Então, segue que

$$
\left|\frac{\partial^{2} v}{\partial y^{2}}\left(x_{m}, y_{m}, z_{m}\right)-\frac{\partial^{2} v}{\partial y^{2}}(0,0, z)\right| \leq\left|\frac{\partial^{2} v}{\partial x^{2}}\left(x_{m}, y_{m}, z_{m}\right)-\frac{\partial^{2} v}{\partial x^{2}}(0,0, z)\right|+g\left(r_{m}, \theta_{m}, z_{m}\right),
$$

onde $g\left(r_{m}, \theta_{m}, z_{m}\right)$ é igual a

$$
\begin{aligned}
& \sum_{j=2}^{\infty}\left|\left[\left(\frac{\mu_{j 0}}{\pi}\right)^{2} J_{0}^{\prime \prime}\left(\frac{\mu_{j 0}}{\pi} r_{m}\right)+\frac{\mu_{j 0}}{\pi r_{m}} J_{0}^{\prime}\left(\frac{\mu_{j 0}}{\pi} r_{m}\right)\right] e^{-\frac{\mu_{j 0}}{\pi} z_{m}}+\left(\frac{\mu_{j 0}}{\pi}\right)^{2} e^{-\frac{\mu_{j 0}}{\pi} z}\right|\left|a_{j 0}\right| \\
+ & \sum_{j, k \geq 1}^{\infty}\left|\left(\frac{\mu_{j k}}{\pi}\right)^{2} J_{k}^{\prime \prime}\left(\frac{\mu_{j k}}{\pi} r_{m}\right)-\frac{k^{2}}{r_{m}^{2}} J_{k}\left(\frac{\mu_{j k}}{\pi} r_{m}\right)+\frac{\mu_{j k}}{\pi r_{m}} J_{k}^{\prime}\left(\frac{\mu_{j k}}{\pi} r_{m}\right)\right| e^{-\frac{\mu_{j k}}{\pi} z_{m}}\left(\left|a_{j k}\right|+\left|b_{j k}\right|\right) .
\end{aligned}
$$

Analogamente, usando a Regra de L'Hospital e o valor das funções de Bessel e de suas 
derivadas em zero, segue que

$$
\lim _{m \rightarrow \infty} \frac{\partial^{2} v}{\partial y^{2}}\left(x_{m}, y_{m}, z_{m}\right)=\frac{\partial^{2} v}{\partial y^{2}}(0,0, z)
$$

e portanto, $\frac{\partial^{2} v}{\partial y^{2}}$ é contínua em $(0,0, z)$, para qualquer $z>0$.

(g) $\frac{\partial^{2} v}{\partial x \partial y}$ é contínua em $(0,0, z)$, para qualquer $z>0$.

De fato, para qualquer $(x, y, z) \in\left(\Omega_{b} \backslash\{(0,0)\}\right) \times(0, \infty)$, sabemos que

$$
\begin{aligned}
\frac{\partial^{2} v}{\partial x \partial y}(x, y, z) & =\cos (2 \theta)\left(\frac{1}{r} \frac{\partial^{2} V}{\partial \theta \partial r}(r, \theta, z)-\frac{1}{r^{2}} \frac{\partial V}{\partial \theta}\right) \\
& -\frac{\sin (2 \theta)}{2}\left(\frac{\partial^{2} V}{\partial r^{2}}(r, \theta, z)+\frac{1}{r^{2}} \frac{\partial^{2} V}{\partial \theta^{2}}(r, \theta, z)+\frac{1}{r} \frac{\partial V}{\partial r}(r, \theta, z)\right) \\
& +\sin (2 \theta) \frac{\partial^{2} V}{\partial r^{2}}(r, \theta, z) .
\end{aligned}
$$

Logo,

$$
\left|\frac{\partial^{2} v}{\partial x \partial y}\left(x_{m}, y_{m}, z_{m}\right)-\frac{\partial^{2} v}{\partial x \partial y}(0,0, z)\right| \leq h\left(r_{m}, \theta_{m}, z_{m}\right),
$$

onde $h\left(r_{m}, \theta_{m}, z_{m}\right)$ é igual a

$$
\begin{array}{ll} 
& \sum_{j=2}^{\infty} \mid\left[-\frac{2}{3}\left(\frac{\mu_{j 2}}{\pi}\right)^{2} J_{2}^{\prime \prime}\left(\frac{\mu_{j 2}}{\pi} r_{m}\right)-\frac{2}{r_{m}} J_{2}\left(\frac{\mu_{j 2}}{\pi} r_{m}\right)\right. \\
+ & \left.\frac{2}{3} \quad\left(\frac{\mu_{j 2}}{\pi}\right)^{2} J_{0}\left(\frac{\mu_{j 2}}{\pi} r_{m}\right)\right] e^{-\frac{\mu_{j 2}}{\pi} z_{m}}-\frac{1}{4}\left(\frac{\mu_{j 2}}{\pi}\right)^{2} e^{-\frac{\mu_{j 2}}{\pi} z}|| b_{j 2} \mid \\
+ & \frac{1}{2} \sum_{j=2}^{\infty}\left|\left(\frac{\mu_{j 2}}{\pi}\right)^{2} J_{0}^{\prime \prime}\left(\frac{\mu_{j 0}}{\pi} r_{m}\right)-\frac{\mu_{j 0}}{\pi r_{m}} J_{0}^{\prime}\left(\frac{\mu_{j 0}}{\pi}\right)\right| e^{-\frac{\mu_{j 0}}{\pi} z_{m}}\left|a_{j 0}\right| \\
+ & \sum_{j=1}^{\infty}\left|\frac{4}{3}\left(\frac{\mu_{j 2}}{\pi}\right)^{2} J_{2}^{\prime \prime}\left(\frac{\mu_{j 2}}{\pi} r_{m}\right)+\frac{4}{r_{m}^{2}} J_{2}\left(\frac{\mu_{j 2}}{\pi} r_{m}\right)-\frac{5}{6}\left(\frac{\mu_{j 2}}{\pi}\right)^{2} J_{0}\left(\frac{\mu_{j 2}}{\pi} r_{m}\right)\right| e^{-\frac{\mu_{j 2}}{\pi} z_{m}}\left(\left|a_{j 2}\right|+\left|b_{j 2}\right|\right) \\
+ & \frac{1}{2} \sum_{\substack{j, k \geq 1 \\
k \neq 2}}^{\infty}\left|\left(\frac{\mu_{j k}}{\pi}\right)^{2} J_{k}^{\prime \prime}\left(\frac{\mu_{j k}}{\pi} r_{m}\right)+\frac{k^{2}}{r_{m}^{2}} J_{k}\left(\frac{\mu_{j k}}{\pi} r_{m}\right)-\frac{\mu_{j k}}{\pi r_{m}} J_{k}^{\prime}\left(\frac{\mu_{j k}}{\pi} r_{m}\right)\right| e^{-\frac{\mu_{j k}}{\pi} z_{m}}\left(\left|a_{j k}\right|+\left|b_{j k}\right|\right) \\
+ & \frac{1}{2} \sum_{\substack{j, k \geq 1 \\
k \neq 2}}^{\infty}\left|\frac{k \mu_{j k}}{\pi r_{m}} J_{k}^{\prime}\left(\frac{\mu_{j k}}{\pi} r_{m}\right)+\frac{k}{r_{m}^{2}} J_{k}\left(\frac{\mu_{j k}}{\pi} r_{m}\right)\right| e^{-\frac{\mu_{j k}}{\pi} z_{m}}\left(\left|a_{j k}\right|+\left|b_{j k}\right|\right) .
\end{array}
$$

Analogamente, pela Regra de L'Hospital e o fato das funções de Bessel e de suas derivadas se anularem no zero, temos que

$$
\lim _{m \rightarrow \infty} \frac{\partial^{2} v}{\partial x \partial y}\left(x_{m}, y_{m}, z_{m}\right)=\frac{\partial^{2} v}{\partial x \partial y}(0,0, z)
$$

e portanto, $\frac{\partial^{2} v}{\partial x \partial y}$ é contínua em $(0,0, z)$, para qualquer $z>0$.

(h) $\frac{\partial^{2} v}{\partial z^{2}}$ é contínua em $(0,0, z)$, para qualquer $z>0$. 
De fato, temos

$$
\begin{aligned}
\left|\frac{\partial^{2} v}{\partial z^{2}}\left(x_{m}, y_{m}, z_{m}\right)-\frac{\partial^{2} v}{\partial z^{2}}(0,0, z)\right| & \leq \sum_{j=2}^{\infty}\left|J_{0}\left(\frac{\mu_{j 0}}{\pi} r_{m}\right) e^{-\frac{\mu_{j 0}}{\pi} z_{m}}-e^{-\frac{\mu_{j 0}}{\pi} z}\right|\left(\frac{\mu_{j 0}}{\pi}\right)^{2}\left|a_{j 0}\right| \\
& +\sum_{j, k \geq 1}^{\infty}\left|J_{k}\left(\frac{\mu_{j k}}{\pi} r_{m}\right)\right|\left(\frac{\mu_{j k}}{\pi}\right)^{2} e^{-\frac{\mu_{j k}}{\pi} z_{m}}\left(\left|a_{j k}\right|+\left|b_{j k}\right|\right) .
\end{aligned}
$$

Como nos itens anteriores, usando o fato das funções de Bessel se anularem no zero, temos que

$$
\lim _{m \rightarrow \infty} \frac{\partial^{2} v}{\partial z^{2}}\left(x_{m}, y_{m}, z_{m}\right)=\frac{\partial^{2} v}{\partial z^{2}}(0,0, z)
$$

Portanto, $\frac{\partial^{2} v}{\partial z^{2}}$ é contínua em $(0,0, z)$, para qualquer $z>0$.

(i) $\frac{\partial^{2} v}{\partial x \partial z}$ e $\frac{\partial^{2} v}{\partial y \partial z}$ são contínuas em $(0,0, z)$, para qualquer $z>0$.

De fato, a demonstração segue análoga aos casos anteriores.

Finalmente, concluímos que $v \in C^{2}\left(\Omega_{b} \times(0, \infty)\right)$.

2. $\Delta v(x, y, z)=0$ em $\Omega_{b} \times(0, \infty)$.

De fato, para qualquer $(x, y, z) \in\left(\Omega_{b} \backslash\{(0,0)\}\right) \times(0, \infty)$, mostrar que

$$
\Delta v(x, y, z)=0
$$

é equivalente a mostrar que

$$
r^{2} \frac{\partial^{2} V}{\partial r^{2}}(r, \theta, z)+r \frac{\partial V}{\partial r}(r, \theta, z)+\frac{\partial^{2} V}{\partial \theta^{2}}(r, \theta, z)+\frac{\partial^{2} V}{\partial z^{2}}(r, \theta, z)=0,
$$

para qualquer $(r, \theta, z) \in(0, \pi) \times \mathbb{R} \times(0, \infty)$. O que é verificado facilmente, através de algumas contas.

Seja $z>0$, então

$$
\begin{aligned}
\Delta v(0,0, z) & =\frac{1}{4} \sum_{j=1}^{\infty} a_{j 2}\left(\frac{\mu_{j 2}}{\pi}\right)^{2} e^{-\frac{\mu_{j 2}}{\pi} z}-\frac{1}{2} \sum_{j=2}^{\infty} a_{j 0}\left(\frac{\mu_{j 0}}{\pi}\right)^{2} e^{-\frac{\mu_{j 0}}{\pi} z} \\
& -\frac{1}{4} \sum_{j=1}^{\infty} a_{j 2}\left(\frac{\mu_{j 2}}{\pi}\right)^{2} e^{-\frac{\mu_{j 2}}{\pi} z}-\frac{1}{2} \sum_{j=2}^{\infty} a_{j 0}\left(\frac{\mu_{j 0}}{\pi}\right)^{2} e^{-\frac{\mu_{j 0}}{\pi} z} \\
& +\sum_{j=2}^{\infty} a_{j 0}\left(\frac{\mu_{j 0}}{\pi}\right)^{2} e^{-\frac{\mu_{j 0}}{\pi} z} \\
& =0 .
\end{aligned}
$$

Portanto, $\Delta v(x, y, z)=0$ em $\Omega_{b} \times(0, \infty)$.

3. $v(\cdot, \cdot, 0)=u(\cdot, \cdot)$ quase sempre em $\Omega_{b}$. 
De fato, seja $u \in D\left(A_{\frac{1}{2}}\right) \subset X, \operatorname{logo}$

$$
u=\sum_{(j, k) \in \mathcal{I}}^{\infty}<u, \varphi_{j k}>\varphi_{j k}
$$

em $L^{2}\left(\Omega_{b}\right)$.

Temos ainda que

$$
\begin{aligned}
\|v\|_{L^{2}\left(\Omega_{b} \times(0, \infty)\right)}^{2} & =\int_{0}^{\infty}<V(\cdot, \cdot, z), V(\cdot, \cdot, z)>_{L^{2}\left(\Omega_{b} \times(0, \infty) ; r\right)} d z \\
& =\int_{0}^{\infty} \sum_{(j, k) \in \mathcal{I}}^{\infty} e^{-\frac{2 \mu_{j k}}{\pi} z}\left|<U, w_{j k}>\right|^{2} d z,
\end{aligned}
$$

onde

(a) Se $k \neq 0$, então

$$
\mu_{j k}>k \geq 1
$$

(b) Se $k=0$, então $j \geq 2$, $\log$ o

$$
\mu_{j 0}>\mu_{20}>\sigma_{10}>\frac{3 \pi}{4}
$$

Logo,

$$
\|v\|_{L^{2}\left(\Omega_{b} \times(0, \infty)\right)}^{2}<\sum_{(j, k) \in \mathcal{I}}^{\infty}\left|<U, w_{j k}>\right|^{2}\left(\int_{0}^{\infty} e^{-\frac{2}{\pi} z} d z\right)<\infty .
$$

Usando o mesmo raciocínio e o Método de Integração por Partes, temos que

$$
\begin{aligned}
\|\nabla v\|_{L^{2}\left(\Omega_{b} \times(0, \infty)\right)}^{2} & =\int_{0}^{\infty} \int_{\Omega_{b}}\left|\nabla_{x y}(x, y, z)\right|^{2} d x d y d z+\int_{0}^{\infty} \int_{\Omega_{b}}\left|v_{z}(x, y, z)\right|^{2} d x d y d z \\
& \leq\left\|v_{z}\right\|_{L^{2}\left(\Omega_{b} \times(0, \infty)\right)}\|v\|_{L^{2}\left(\Omega_{b} \times(0, \infty)\right)}+\left\|v_{z}\right\|_{L^{2}\left(\Omega_{b} \times(0, \infty)\right)}^{2} \\
& <\infty
\end{aligned}
$$

pois

$$
\left\|v_{z}\right\|_{L^{2}\left(\Omega_{b} \times(0, \infty)\right)}^{2}<\sum_{(j, k) \in \mathcal{I}}^{\infty}\left(\frac{\mu_{j k}}{\pi}\right)^{2}\left|<U, w_{j k}>\right|^{2}\left(\int_{0}^{\infty} e^{-\frac{2}{\pi} z} d z\right)<\infty .
$$

Sabemos que $v \in L^{2}\left(\Omega_{b} \times(0, \infty)\right)$ e como

$$
L_{l o c}^{2}\left(\Omega_{b} \times(0, \infty)\right) \subset L_{l o c}^{1}\left(\Omega_{b} \times(0, \infty)\right) \hookrightarrow D^{\prime}\left(\Omega_{b} \times(0, \infty)\right)
$$

segue que $v \in D^{\prime}\left(\Omega_{b} \times(0, \infty)\right)$.

Portanto, $v \in H^{1}\left(\Omega_{b} \times(0, \infty)\right)$ e analogamente aos casos dos domínios $\Omega_{i}$ e $\Omega_{q}$ temos que

$$
v(\cdot, \cdot, 0)=u(\cdot, \cdot)
$$


quase sempre em $\Omega_{b}$.

4. $\frac{\partial v}{\partial n}(x, y, z)=0$ em $\partial \Omega_{b} \times[0, \infty)$.

De fato, observe primeiramente, que para provarmos este item, basta verificarmos que

$$
\frac{\partial V}{\partial r}(\pi, \theta, z)=0
$$

para qualquer $\theta \in \mathbb{R}, z \geq 0$, pois aqui não temos o problema no ponto $(0,0, z)$, para qualquer $z \geq 0$.

Seja $\theta \in \mathbb{R}$ e $z>0$. Então,

$$
\begin{aligned}
\frac{\partial V}{\partial r}(\pi, \theta, z) & =\sum_{j, k \geq 1}^{\infty} \frac{\mu_{j k}}{\pi} J_{k}^{\prime}\left(\mu_{j k}\right) e^{-\frac{\mu_{j k}}{\pi} z}\left[a_{j k} \cos (k \theta)+b_{j k} \sin (k \theta)\right] \\
& +\sum_{j=1}^{\infty} \frac{\mu_{j 0}}{\pi} J_{0}^{\prime}\left(\mu_{j 0}\right) e^{-\frac{\mu_{j 0}}{\pi} z} a_{j 0} \\
& =0 .
\end{aligned}
$$

Por outro lado, temos que

$$
\frac{\partial V}{\partial r}(\pi, \theta, 0)=\lim _{z \rightarrow 0^{+}} \frac{\partial V}{\partial r}(\pi, \theta, z)=0 .
$$

Portanto, $\frac{\partial v}{\partial n}(x, y, z)=0$ em $\partial \Omega_{b} \times[0, \infty)$.

5

$$
\int_{\Omega_{b}} v(x, y, z) d x d y=0
$$

para qualquer $z \geq 0$.

De fato, seja $z>0$, então sabendo que

$$
\int_{\Omega_{b}} v(x, y, z) d x d y=\int_{0}^{2 \pi} \int_{0}^{\pi} r V(r, \theta, z) d r d \theta
$$

temos,

$$
\begin{aligned}
\int_{\Omega_{b}} v(x, y, z) d x d y & =\sum_{j=2}^{\infty} e^{-\frac{\mu_{j 0}}{\pi} z} a_{j 0}\left(2 \pi \int_{0}^{\pi} r J_{0}\left(\frac{\mu_{j 0}}{\pi} r\right) d r\right) \\
& +\sum_{j, k \geq 1}^{\infty} e^{-\frac{\mu_{j k}}{\pi} z}\left(\int_{0}^{2 \pi}\left(a_{j k} \cos (k \theta)+b_{j k} \sin (k \theta)\right) d \theta\right)\left(\int_{0}^{\pi} r J_{k}\left(\frac{\mu_{j k}}{\pi} r\right) d r\right) \\
& =0 .
\end{aligned}
$$

O caso $z=0$ segue do fato de $u$ ter média zero e coincidir quase sempre com $v(\cdot, \cdot, 0)$ em $\Omega_{q}$. 
Portanto,

$$
\int_{\Omega_{b}} v(x, y, z) d x d y=0
$$

para qualquer $z \geq 0$.

6. $\lim _{z \rightarrow \infty}\|v(\cdot, \cdot, z)\|_{L^{2}\left(\Omega_{b}\right)}=0$.

De fato, temos que

$$
\lim _{z \rightarrow \infty} \int_{\Omega_{b}}|v(x, y, z)|^{2} d x d y=\lim _{z \rightarrow \infty} \int_{0}^{2 \pi} \int_{0}^{\pi} r|V(r, \theta, z)|^{2} d r d \theta
$$

onde

$$
\int_{0}^{2 \pi} \int_{0}^{\pi} r|V(r, \theta, z)|^{2} d r d \theta \leq\left(\sum_{(j, k) \in \mathcal{I}}^{\infty}\left|<U, w_{j k}>\right|^{2}\right) e^{-\frac{2}{\pi} z}
$$

para qualquer $z>0$.

Logo,

$$
\lim _{z \rightarrow \infty} \int_{0}^{2 \pi} \int_{0}^{\pi} r|V(r, \theta, z)|^{2} d r d \theta=0
$$

Portanto, $\lim _{z \rightarrow \infty}\|v(\cdot, \cdot, z)\|_{L^{2}\left(\Omega_{b}\right)}=0$.

7. $\lim _{z \rightarrow \infty}\left\|v_{z}(\cdot, \cdot, z)\right\|_{L^{2}\left(\Omega_{b}\right)}=0$.

De fato, este item é análogo ao item anterior.

Finalmente, concluímos que $v$ é solução clássica de 4.8. A unicidade da solução é análoga ao caso do domínio $\Omega_{i}$.

Agora que temos a solução de (4.8), podemos exibir a solução de (4.1) no lema a seguir.

Lema 10. Considere $\tilde{v}: \mathbb{R}_{+}^{3} \longrightarrow \mathbb{R}$ como sendo a extensão radial de $v$, $4 \pi$-periódica e simétrica em relação ao bordo de $\Omega_{b}$, isto é

$$
\tilde{V}(\alpha, \theta, z)=\left\{\begin{array}{lll}
\widehat{V}(\alpha-4 k \pi, \theta, z) & \text { se } & (4 k-1) \pi \leq \alpha \leq(4 k+1) \pi \\
\widehat{V}(-\alpha+2(2 k-1) \pi, \theta, z) & \text { se } \quad(4 k-3) \pi \leq \alpha \leq(4 k-1) \pi
\end{array}\right.
$$

onde $\widehat{V}(\alpha, \theta, z)=v(\alpha \cos \theta, \alpha \sin \theta, z) e \tilde{V}(\alpha, \theta, z)=\tilde{v}(\alpha \cos \theta, \alpha \sin \theta, z)$, para qualquer $\alpha, \theta \in \mathbb{R}, z>0$. Então, ṽ é a única solução clássica de (4.1).

Demonstração. Vejamos que $\tilde{v}$ satisfaz a Definição 14.

1. $\tilde{v} \in C^{2}\left(\mathbb{R}_{+}^{3}\right)$.

De fato, pelo teorema anterior temos que $v \in C^{2}\left(\Omega_{b} \times(0, \infty)\right)$, logo pelo Lema 9. já sabemos que $\tilde{v} \in C\left(\mathbb{R}_{+}^{3}\right)$. Também pela periodicidade temos que as derivadas de $\tilde{v}$ são contínuas em $\mathbb{R}_{+}^{3}$, exceto possivelmente, nos pontos

$$
(x, y, z)=(m \pi \cos \theta, m \pi \sin \theta, z)
$$


$\operatorname{com} m \in \mathbb{Z}^{*}$.

Para verificarmos a continuidade das derivadas de $\tilde{v}$ nestes pontos, usando a periodicidade e simetria, basta verificarmos a continuidade das derivadas nos pontos $( \pm \pi \cos \theta, \pm \pi \sin \theta, z)$.

Seja $(x, y, z) \in \mathbb{R}_{+}^{3}$, tal que $(x, y, z)=( \pm \pi \cos \theta, \pm \pi \sin \theta, z)$, logo, como já vimos

$$
\begin{aligned}
\frac{\partial \tilde{v}}{\partial x}(x, y, z) & =\quad \cos \theta \frac{\partial \tilde{V}}{\partial \alpha}( \pm \pi, \theta, z) \mp \frac{1}{\pi} \sin \theta \frac{\partial \tilde{V}}{\partial \theta}( \pm \pi, \theta, z), \\
\frac{\partial \tilde{v}}{\partial y}(x, y, z) & =\quad \sin \theta \frac{\partial \tilde{V}}{\partial \alpha}( \pm \pi, \theta, z) \pm \frac{1}{\pi} \cos \theta \frac{\partial \tilde{V}}{\partial \theta}( \pm \pi, \theta, z), \\
\frac{\partial^{2} \tilde{v}}{\partial y^{2}}(x, y, z) & =\quad \frac{1}{\pi^{2}} \frac{\partial^{2} \tilde{V}}{\partial \theta^{2}}( \pm \pi, \theta, z) \pm \frac{1}{\pi} \frac{\partial \tilde{V}}{\partial \alpha}( \pm \pi, \theta, z), \\
\frac{\partial^{2} \tilde{v}}{\partial x \partial y}(x, y, z) & = \pm \frac{1}{\pi} \frac{\partial^{2} \tilde{V}}{\partial \theta \partial \alpha}( \pm \pi, \theta, z)-\frac{1}{\pi^{2}} \frac{\partial \tilde{V}}{\partial \theta}( \pm \pi, \theta, z), \\
\frac{\partial^{2} \tilde{v}}{\partial x^{2}}(x, y, z) & =\frac{\partial^{2} \tilde{V}}{\partial \alpha^{2}}( \pm \pi, \theta, z) \\
\frac{\partial^{2} \tilde{v}}{\partial x \partial z}(x, y, z) & =-\frac{\partial^{2} \tilde{V}}{\partial \alpha \partial z}( \pm \pi, \theta, z) \\
\frac{\partial^{2} \tilde{v}}{\partial y \partial z}(x, y, z) & =\quad \frac{\partial^{2} \tilde{V}}{\partial \theta \partial z}( \pm \pi, \theta, z) .
\end{aligned}
$$

Vimos anteriormente que $v \in C^{2}\left(\Omega_{b} \times(0, \infty)\right)$. Usando os mesmos argumentos temos que $v \in C^{2}\left(\overline{\Omega_{b}} \times(0, \infty)\right)$, então $V$ é $C^{2} \operatorname{nos}$ pontos $(\pi, \theta, z)$, para qualquer $\theta \in \mathbb{R}, z>0$. Mas, $\widehat{V}$ e suas derivadas coincidem com $V$ e suas derivadas em $(\pi, \theta, z), \operatorname{logo} \widehat{V}$ é $C^{2} \operatorname{nos}$ pontos $(\pi, \theta, z)$, para qualquer $\theta \in \mathbb{R}, z>0$.

Assim,

$$
\begin{aligned}
\lim _{h \rightarrow 0^{+}} \frac{\tilde{V}(\pi, \theta+h, z)-\tilde{V}(\pi, \theta, z)}{h} & =\frac{\partial \widehat{V}}{\partial \theta}(\pi, \theta, z) \\
\lim _{h \rightarrow 0^{-}} \frac{\tilde{V}(\pi, \theta+h, z)-\tilde{V}(\pi, \theta, z)}{h} & =\frac{\partial \widehat{V}}{\partial \theta}(\pi, \theta, z),
\end{aligned}
$$

então, existe $\frac{\partial \tilde{V}}{\partial \theta}(\pi, \theta, z)$ e

$$
\frac{\partial \tilde{V}}{\partial \theta}(\pi, \theta, z)=\frac{\partial \widehat{V}}{\partial \theta}(\pi, \theta, z)=-\sum_{(j, k) \geq 1}^{\infty} k J_{k}\left(\mu_{j k}\right) e^{-\frac{\mu_{j k}}{\pi} z}\left(-a_{j k} \sin (k \theta)+b_{j k} \cos (k \theta)\right)
$$

Portanto, $\frac{\partial \tilde{V}}{\partial \theta}$ é contínua em $(\pi, \theta, z)$, para qualquer $\theta \in \mathbb{R}, z>0$. Analogamente, verificamos 
a continuidade de $\frac{\partial \tilde{V}}{\partial \alpha}$ em $(\pi, \theta, z)$, para qualquer $\theta \in \mathbb{R}, z>0$. A continuidade nos pontos $(-\pi, \theta, z)$ é verificada somente observando, que estes pontos coincidem com os pontos $(\pi, \theta+\pi, z)$, para qualquer $\theta \in \mathbb{R}, z>0$, nos quais já vimos a continuidade. Portanto, $\tilde{v} \in C^{1}\left(\mathbb{R}_{+}^{3}\right)$.

Usando o mesmo raciocínio, temos

$$
\begin{aligned}
\lim _{h \rightarrow 0^{+}} \frac{\frac{\partial \tilde{V}}{\partial \alpha}(\pi, \theta+h, z)-\frac{\partial \tilde{V}}{\partial \alpha}(\pi, \theta, z)}{h} & =\frac{\partial^{2} \widehat{V}}{\partial \theta \partial \alpha}(\pi, \theta, z) \\
\lim _{h \rightarrow 0^{-}} \frac{\frac{\partial \tilde{V}}{\partial \alpha}(\pi, \theta+h, z)-\frac{\partial \tilde{V}}{\partial \alpha}(\pi, \theta, z)}{h} & =\frac{\partial^{2} \widehat{V}}{\partial \theta \partial \alpha}(\pi, \theta, z),
\end{aligned}
$$

logo, existe $\frac{\partial^{2} \tilde{V}}{\partial \theta \partial \alpha}(\pi, \theta, z)$ e

$$
\frac{\partial^{2} \tilde{V}}{\partial \theta \partial \alpha}(\pi, \theta, z)=\frac{\partial^{2} \widehat{V}}{\partial \theta \partial \alpha}(\pi, \theta, z)
$$

Já a continuidade é válida pois,

$$
\begin{aligned}
\lim _{\alpha \rightarrow \pi^{+}} \frac{\partial^{2} \tilde{V}}{\partial \theta \partial \alpha}(\alpha, \theta, z) & =-\frac{\partial^{2} \widehat{V}}{\partial \theta \partial \alpha}(\alpha, \theta, z) \\
\lim _{\alpha \rightarrow \pi^{-}} \frac{\partial^{2} \tilde{V}}{\partial \theta \partial \alpha}(\alpha, \theta, z) & =\frac{\partial^{2} \widehat{V}}{\partial \theta \partial \alpha}(\alpha, \theta, z),
\end{aligned}
$$

onde $\frac{\partial^{2} \widehat{V}}{\partial \theta \partial \alpha}(\pi, \theta, z)=\frac{\partial^{2} V}{\partial \theta \partial r}(\pi, \theta, z) \mathrm{e}$

$$
\frac{\partial^{2} V}{\partial \theta \partial r}(\pi, \theta, z)=\sum_{j, k \geq 1}^{\infty} \frac{k \mu_{j k}}{\pi} J_{k}^{\prime}\left(\mu_{j k}\right) e^{-\frac{\mu_{j k}}{\pi} z}\left(-a_{j k} \sin (k \theta)+b_{j k} \cos (k \theta)\right)=0 .
$$

Portanto,

$$
\lim _{\alpha \rightarrow \pi} \frac{\partial^{2} \tilde{V}}{\partial \theta \partial \alpha}(\alpha, \theta, z)=\frac{\partial^{2} \tilde{V}}{\partial \theta \partial \alpha}(\pi, \theta, z)
$$

para qualquer $\theta \in \mathbb{R}, z>0$. Assim, temos que $\frac{\partial^{2} \tilde{V}}{\partial \theta \partial \alpha}$ é contínua em $(\pi, \theta, z)$.

Analogamente, verificamos a continuidade de $\frac{\partial^{2} \tilde{V}}{\partial \theta^{2}}, \frac{\partial^{2} \tilde{V}}{\partial \alpha^{2}}, \frac{\partial^{2} \tilde{V}}{\partial \alpha \partial z}$ e $\frac{\partial^{2} \tilde{V}}{\partial \theta \partial z}$ em $(\pi, \theta, z)$, para qualquer $\theta \in \mathbb{R}, z>0$. Também podemos facilmente verificar a suavidade das derivadas de $\tilde{V}$ em relação a variável z. Portanto, $\tilde{v} \in C^{2}\left(\mathbb{R}_{+}^{3}\right)$.

2.

$$
\tilde{V}(\alpha+4 k \pi, \theta, z)=\tilde{V}(\alpha, \theta, z) \quad \text { e } \quad \tilde{V}(-\alpha-2 \pi, \theta, z)=\tilde{V}(\alpha, \theta, z)
$$

para qualquer $k \in \mathbb{Z}, \alpha, \theta \in \mathbb{R}$ e $z \geq 0$. 
De fato, para $z>0$ a demonstração é idêntica a demonstração do Lema 9. Para $z=0$, basta usarmos as propriedades de limite, pois

$$
\tilde{V}(\alpha+4 k \pi, \theta, 0)=\lim _{z \rightarrow 0^{+}} \tilde{V}(\alpha+4 k \pi, \theta, z)=\lim _{z \rightarrow 0^{+}} \tilde{V}(\alpha, \theta, z)=\tilde{V}(\alpha, \theta, 0),
$$

para qualquer $k \in \mathbb{Z}, \alpha, \theta \in \mathbb{R}$.

Analogamente, temos $\tilde{V}(-\alpha-2 \pi, \theta, z)=\tilde{V}(\alpha, \theta, z)$, para qualquer $k \in \mathbb{Z}, \alpha, \theta \in \mathbb{R}$ e $z \geq 0$.

3. $\tilde{v}(\cdot, \cdot, 0)=\tilde{u}(\cdot, \cdot)$ quase sempre em $\mathbb{R}^{2}$.

De fato, segue do fato de $\tilde{v}$ e $v$ coincidirem em $\Omega_{b} \times(0, \infty)$ e

$$
v(\cdot, \cdot, 0)=u(\cdot, \cdot),
$$

quase sempre em $\Omega_{b}$.

4. $\Delta \tilde{v}(x, y, z)=0 \mathrm{em} \mathbb{R}_{+}^{3}$.

De fato, como

$$
\Delta v(x, y, z)=0 \quad \text { em } \quad \Omega_{b} \times(0, \infty)
$$

segue pela periodicidade que

$$
\Delta \tilde{v}(x, y, z)=0 \quad \text { em } \quad \mathbb{R}_{+}^{3},
$$

exceto possivelmente, nos pontos $(x, y, z)=(m \pi \cos \theta, m \pi \sin \theta, z), m \in \mathbb{Z}^{*}$.

Seja $(x, y, z)=(\pi \cos \theta, \pi \sin \theta, z), \operatorname{com} \theta \in \mathbb{R}$ e $z>0$ arbitrários. Assim,

$$
\begin{aligned}
\Delta \tilde{v}(x, y, z) & =\tilde{V}_{\alpha \alpha}(\pi, \theta, z)+\frac{1}{\pi} \tilde{V}_{\alpha}(\pi, \theta, z)+\frac{1}{\pi^{2}} \tilde{V}_{\theta \theta}(\pi, \theta, z) \\
& =V_{\alpha \alpha}(\pi, \theta, z)+\frac{1}{\pi} V_{\alpha}(\pi, \theta, z)+\frac{1}{\pi^{2}} V_{\theta \theta}(\pi, \theta, z) \\
& =\frac{1}{\pi^{2}} \sum_{(j, k) \in \mathcal{I}}^{\infty} T_{j k}\left(\mu_{j k}\right) e^{-\frac{\mu_{j k}}{\pi}} z\left[a_{j k} \cos (k \theta)+b_{j k} \sin (k \theta)\right]
\end{aligned}
$$

onde $T_{j k}\left(\mu_{j k}\right)=\mu_{j k}^{2} J_{k}^{\prime \prime}\left(\mu_{j k}\right)+\mu_{j k} J_{k}^{\prime}\left(\mu_{j k}\right)+\left(\mu_{j k}^{2}-k^{2}\right) J_{k}\left(\mu_{j k}\right)=0$.

Logo,

$$
\Delta \tilde{v}(x, y, z)=0
$$

para $(x, y, z)=(\pi \cos \theta, \pi \sin \theta, z)$.

Por outro lado, se $(x, y, z)=(-\pi \cos \theta,-\pi \sin \theta, z)$, temos que

$$
\Delta \tilde{v}(x, y, z)=\Delta \tilde{v}(\pi \cos (\theta+\pi), \pi \sin (\theta+\pi), z)=0 .
$$

Portanto, $\Delta \tilde{v}(x, y, z)=0$ em $\mathbb{R}_{+}^{3}$. 
5.

$$
\int_{\Omega_{b}} \tilde{v}(x, y, z) d x d y=0
$$

para qualquer $z \geq 0$.

De fato,

$$
\int_{\Omega_{b}} \tilde{v}(x, y, z) d x d y=\int_{\Omega_{b}} v(x, y, z) d x d y=0
$$

para qualquer $z \geq 0$.

6.

$$
\lim _{z \rightarrow \infty}\|\tilde{v}(\cdot, \cdot, z)\|_{L^{2}\left(\Omega_{b}\right)}=\lim _{z \rightarrow \infty}\left\|\tilde{v}_{z}(\cdot, \cdot, z)\right\|_{L^{2}\left(\Omega_{b}\right)}=0 .
$$

De fato,

$$
\lim _{z \rightarrow \infty}\|\tilde{v}(\cdot, \cdot, z)\|_{L^{2}\left(\Omega_{b}\right)}=\lim _{z \rightarrow \infty}\|v(\cdot, \cdot, z)\|_{L^{2}\left(\Omega_{b}\right)}=0
$$

e

$$
\lim _{z \rightarrow \infty}\left\|\tilde{v}_{z}(\cdot, \cdot, z)\right\|_{L^{2}\left(\Omega_{b}\right)}=\lim _{z \rightarrow \infty}\|v(\cdot, \cdot, z)\|_{L^{2}\left(\Omega_{b}\right)}=0 .
$$

Portanto, $\tilde{v}$ é solução clássica de (4.1) e a unicidade segue analogamente ao caso do domínio $\Omega_{i}$.

Agora, podemos finalmente definir o operador $A_{\frac{1}{2}}$ para o caso $\Omega_{b}$.

Definição 15. Seja

$$
D\left(A_{\frac{1}{2}}\right)=\left\{u \in H^{s}\left(\Omega_{b}\right)\left|s>2, \quad \frac{\partial u}{\partial n}\right|_{\partial \Omega_{b}}=0 e \int_{\Omega_{b}} u(x, y) d x d y=0\right\}
$$

Definimos o seguinte operador

$$
\begin{aligned}
A_{\frac{1}{2}}: D\left(A_{\frac{1}{2}}\right) \subset X & \longrightarrow X \\
u & \longmapsto-\left.\left(\tilde{v}_{z}(\cdot, \cdot, 0)\right)\right|_{\Omega_{b}},
\end{aligned}
$$

onde ṽ é a única solução clássica (4.1), para o caso $\Omega_{b}$.

Analogamente aos domínios $\Omega_{i}$ e $\Omega_{q}$, o operador $A_{\frac{1}{2}}$ está bem definido e

$$
A_{\frac{1}{2}} u=\sum_{(j, k) \in \mathcal{I}}^{\infty} \lambda_{j k}^{\frac{1}{2}}<u, \varphi_{j k}>\varphi_{j k}
$$

em $L^{2}\left(\Omega_{b}\right)$

Nosso objetivo agora, é estender o operador $A_{\frac{1}{2}}$ e verificar que esta extensão coincide com o operador $\left(-\Delta_{N}\right)^{\frac{1}{2}}$ em $\Omega_{b}$.

Definição 16. Seja

$$
Y=\left\{u \in X\left|\sum_{(j, k) \in \mathcal{I}}^{\infty} \lambda_{j k}\right|<U, w_{j k}>\left.\right|^{2}<\infty\right\}
$$


Definimos $B_{\frac{1}{2}}: Y \subset X \longrightarrow X$ tal que

$$
B_{\frac{1}{2}} u=\sum_{(j, k) \in \mathcal{I}}^{\infty} \lambda_{j k}^{\frac{1}{2}}<u, \varphi_{j k}>\varphi_{j k}
$$

onde $\varphi_{j k}$ são as autofunções do operador $-\Delta_{N}$ em $\Omega_{b}$ e $\lambda_{j k}$ são os respectivos autovalores.

Lema 11. O operador $B_{\frac{1}{2}}$ está bem definido e é uma extensão de $A_{\frac{1}{2}}$.

Demonstração. Seja $u \in Y$, temos

$$
\sum_{(j, k) \in \mathcal{I}}^{\infty} \lambda_{j k}\left|<U, w_{j k}>\right|^{2}<\infty
$$

logo, a série

$$
\sum_{(j, k) \in \mathcal{I}}^{\infty} \lambda_{j k}^{\frac{1}{2}}<U, w_{j k}>w_{j k}
$$

converge em $L^{2}((0, \pi) \times(0,2 \pi) ; r)$ e com isso $B_{\frac{1}{2}} u \in L^{2}\left(\Omega_{b}\right)$.

Considere a soma parcial

$$
s_{m n}=\sum_{j=2}^{m} \lambda_{j 0}^{\frac{1}{2}}<U, w_{j 0}>w_{j 0}+\sum_{j=1}^{m} \sum_{k=1}^{n} \lambda_{j k}^{\frac{1}{2}}<U, w_{j k}>w_{j k} .
$$

Como

$$
\int_{0}^{2 \pi} \int_{0}^{\pi} r s_{m n}(r, \theta) d r d \theta=0
$$

para qualquer $(m, n) \in \mathcal{I}$, segue pela Desigualdade de Hölder, que

$$
\begin{aligned}
\left|\int_{\Omega_{b}} B_{\frac{1}{2}} u(x, y) d x d y\right| & \leq \int_{0}^{2 \pi} \int_{0}^{\pi} r\left|B_{\frac{1}{2}} U(r, \theta)-s_{m n}(r, \theta)\right| d r d \theta \\
& \leq M\left\|B_{\frac{1}{2}} U-s_{m n}\right\|_{L^{2}((0, \pi) \times(0,2 \pi) ; r) \stackrel{m, n \rightarrow \infty}{\longrightarrow}}^{\longrightarrow} .
\end{aligned}
$$

Então, $B_{\frac{1}{2}} u \in X$ e pela unicidade da série de Fourier-Bessel, segue que $B_{\frac{1}{2}}$ está bem definido.

A prova de que o operador $B_{\frac{1}{2}}$ é uma extensão do operador $A_{\frac{1}{2}}$ é análoga aos casos dos domínios $\Omega_{i}$ e $\Omega_{q}$.

Logo, com o operador $B_{\frac{1}{2}}$ bem definido, temos o seguinte teorema.

Teorema 11. O operador $B_{\frac{1}{2}}$ coincide com o operador $\left(-\Delta_{N}\right)^{\frac{1}{2}}$ em $\Omega_{b}$, ou seja,

$$
<u, B_{\frac{1}{2}} u>\geq 0
$$

para qualquer $u \in D\left(B_{\frac{1}{2}}\right) e$

$$
B_{\frac{1}{2}} \circ B_{\frac{1}{2}} u=-\Delta_{N} u,
$$

para qualquer $u \in D\left(-\Delta_{N}\right)$. 
Demonstração. A demonstração é análoga aos casos dos domínios $\Omega_{i}$ e $\Omega_{q}$. 


\section{Capítulo 5}

\section{Aplicação}

Neste capítulo estudaremos a existência de solução fraca não nula do problema não local

$$
\left(-\Delta_{N}\right)^{\frac{1}{2}} u=u^{p}
$$

no intervalo e no quadrado, onde $\left(-\Delta_{N}\right)^{\frac{1}{2}}$ é a raiz quadrada do operador Laplaciano com condição de fronteira de Neumann, que coincide com o operador $B_{\frac{1}{2}}$ definido no capítulo anterior.

Consideraremos

$$
p=2+\frac{1}{s},
$$

com $s>1$ ímpar, quando o domínio for $\Omega_{q}$, e

$$
p=\bar{p}+\frac{1}{s},
$$

com $\bar{p}$ par e $s \geq 1$ ímpar, quando o domínio for $\Omega_{i}$.

Para que o problema não local (5.1) faça sentido, precisamos inicialmente encontrar uma solução $u$ tal que

$$
\int_{\Omega} u^{p}(x) d x=0
$$

nos domínios $\Omega_{i}$ e $\Omega_{q}$. Para que isto ocorra, vamos procurar soluções satisfazendo algumas condições, que serão exibidas nas próximas seções, de acordo com o domínio.

O estudo da existência de solução fraca não nula do problema não local (5.1), será feito através do estudo da existência de solução fraca não nula de um problema local.

Quando o domínio for $\Omega_{q}$, o problema local correspondente é

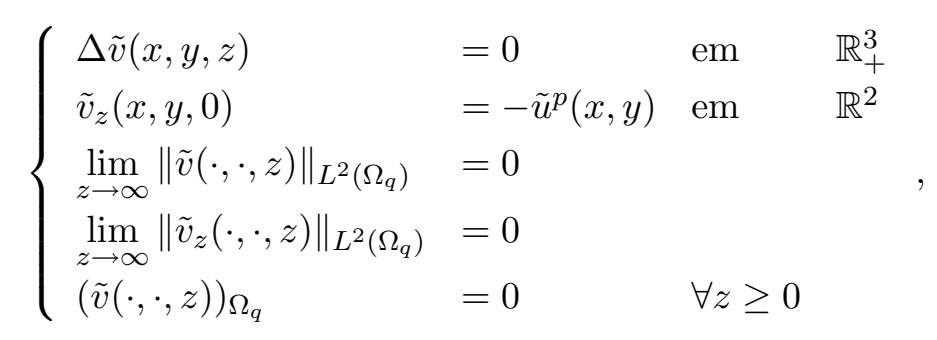

onde $\tilde{v}$ é par, periódica nas variáveis $x$ e $y$, e $\tilde{u}$ é a extensão par, periódica de $u$ à $\mathbb{R}^{2}$. 
Já para o domínio $\Omega_{i}$, o problema local correspondente é

$$
\left\{\begin{array}{llll}
\Delta \tilde{v}(x, z) & =0 & \text { em } & \mathbb{R}_{+}^{2} \\
\tilde{v}_{z}(x, 0) & =-\tilde{u}^{p}(x) & \text { em } \quad \mathbb{R} \\
\lim _{z \rightarrow \infty}\|\tilde{v}(\cdot, z)\|_{L^{2}\left(\Omega_{i}\right)} & =0 & & \\
\lim _{z \rightarrow \infty}\left\|\tilde{v}_{z}(\cdot, z)\right\|_{L^{2}\left(\Omega_{i}\right)} & =0 & & \\
(\tilde{v}(\cdot, z))_{\Omega_{i}} & =0 & \forall z \geq 0
\end{array},\right.
$$

onde $\tilde{v}$ é par, periódica na variável $x$ e $\tilde{u}$ é uma extensão par, periódica de $u$ à $\mathbb{R}$.

Este capítulo será dividido em três seções. Nas duas primeiras seções, daremos a definição de solução fraca para os casos de domínios $\Omega_{i}$ e $\Omega_{q}$, respectivamente. Na terceira seção, veremos a existência de solução fraca não nula do problema não linear (5.1), a qual se resume na verificação das hipóteses do Teorema do Multiplicador de Lagrange em espaços lineares topológicos. Esta terceira seção será para os dois casos de domínios, diferenciando quando necessário.

Para facilitar, usaremos sempre $B_{\frac{1}{2}}$ para denotar o operador $\left(-\Delta_{N}\right)^{\frac{1}{2}}$.

\subsubsection{Solução fraca no intervalo}

Vejamos uma motivação para definição de solução fraca de (5.1).

Seja $u \in D\left(A_{\frac{1}{2}}\right)$, logo para qualquer $x \in \Omega_{i}$,

$$
B_{\frac{1}{2}} u(x)=A_{\frac{1}{2}} u(x)=-\tilde{v}_{z}(x, 0),
$$

onde $\tilde{v}$ é solução clássica de

$$
\left\{\begin{array}{lllll}
\Delta \tilde{v}(x, z) & = & 0 & \text { em } & \mathbb{R}_{+}^{2} \\
\tilde{v}(x, 0) & = & \tilde{u}(x) & \text { em } \quad & \mathbb{R} \\
\lim _{z \rightarrow \infty}\|\tilde{v}(\cdot, z)\|_{L^{2}\left(\Omega_{i}\right)} & = & 0 & & \\
\lim _{z \rightarrow \infty}\left\|\tilde{v}_{z}(\cdot, z)\right\|_{L^{2}\left(\Omega_{i}\right)} & = & 0 & \\
(\tilde{v}(\cdot, z))_{\Omega_{i}} & = & 0 & \forall z \geq 0
\end{array},\right.
$$

tal que $\tilde{u}$ é a extensão par, $2 \pi$-periódica de $u$ em $\mathbb{R}$.

Considere $\tilde{\varphi} \in H_{p e r}^{1}\left(\mathbb{R}_{+}^{2}\right)$, par quase sempre na variável $x$, tal que $\tilde{\varphi}(x, 0)=\varphi(x)$,

$$
\int_{\Omega_{i}} \tilde{\varphi}(x, z) d x=0
$$

para qualquer $z \geq 0$ e $\lim _{z \rightarrow \infty}\|\tilde{\varphi}(\cdot, z)\|_{L^{2}\left(\Omega_{i}\right)}=\lim _{z \rightarrow \infty}\left\|\tilde{\varphi}_{z}(\cdot, z)\right\|_{L^{2}\left(\Omega_{i}\right)}=0$.

Logo,

$$
\int_{\Omega_{i}} u^{p}(x) \varphi(x) d x=\int_{\Omega_{i}} B_{\frac{1}{2}} u(x) \varphi(x) d x=-\int_{\Omega_{i}} \tilde{v}_{z}(x, 0) \varphi(x) d x .
$$

Como $\tilde{v}$ é solução clássica de (5.4), usando o Método de Integração por Partes, temos para qualquer 
$T>0$ que

$$
\begin{aligned}
\int_{0}^{T} \int_{\Omega_{i}} \nabla \tilde{v}(x, z) \nabla \tilde{\varphi}(x, z) d x d z= & -\int_{\Omega_{i}} \tilde{v}_{z}(x, 0) \tilde{\varphi}(x, 0) d x \\
& +\int_{\Omega_{i}} \tilde{v}_{z}(x, T) \tilde{\varphi}(x, T) d x .
\end{aligned}
$$

Fazendo $T \rightarrow \infty$ temos

$$
\begin{aligned}
\int_{0}^{\infty} \int_{\Omega_{i}} \nabla \tilde{v}(x, z) \nabla \tilde{\varphi}(x, z) d x d z= & -\int_{\Omega_{i}} \tilde{v}_{z}(x, 0) \tilde{\varphi}(x, 0) d x d y \\
& +\lim _{T \rightarrow \infty} \int_{\Omega_{i}} \tilde{v}_{z}(x, T) \tilde{\varphi}(x, T) d x .
\end{aligned}
$$

Usando a Desigualdade de Hölder temos

$$
\begin{aligned}
\left|\int_{\Omega_{i}} \tilde{v}_{z}(x, T) \tilde{\varphi}(x, T) d x\right| & \leq\left\|\tilde{v}_{z}(\cdot, T)\right\|_{L^{2}\left(\Omega_{i}\right)}\|\tilde{\varphi}(\cdot, T)\|_{L^{2}\left(\Omega_{i}\right)} \\
& \Longrightarrow \lim _{T \rightarrow \infty} \int_{\Omega_{i}} \tilde{v}_{z}(x, T) \tilde{\varphi}(x, T) d x=0 .
\end{aligned}
$$

Portanto, de (5.5) e (5.6) segue que

$$
\int_{0}^{\infty} \int_{\Omega_{i}} \nabla \tilde{v}(x, z) \nabla \tilde{\varphi}(x, z) d x d z=\int_{\Omega_{i}} \tilde{u}^{p}(x) \tilde{\varphi}(x, 0) d x
$$

para qualquer $\tilde{\varphi} \in H_{\text {per }}^{1}\left(\mathbb{R}_{+}^{2}\right)$, par quase sempre na variável $x$,

$$
\int_{\Omega_{i}} \tilde{\varphi}(x, z) d x=0
$$

para qualquer $z \geq 0$ tal que $\lim _{T \rightarrow \infty}\left\|\tilde{\varphi}_{z}(\cdot, T)\right\|_{L^{2}\left(\Omega_{i}\right)}=\lim _{T \rightarrow \infty}\|\tilde{\varphi}(\cdot, T)\|_{L^{2}\left(\Omega_{i}\right)}=0$.

Com isso temos a seguinte definição de solução fraca de (5.1) para $\Omega_{i}$.

Definição 17. Dizemos que u é solução fraca de (5.1) se $u=\left.(\tilde{v}(\cdot, 0))\right|_{\Omega_{i}}$, onde $\tilde{v} \in H_{\text {per }}^{1}\left(\mathbb{R}_{+}^{2}\right)$, é par quase sempre na variável $x$,

$$
\int_{\Omega_{i}} \tilde{v}(x, z) d x=0
$$

para qualquer $z \geq 0, \lim _{T \rightarrow \infty}\|\tilde{v}(\cdot, T)\|_{L^{2}\left(\Omega_{i}\right)}=\lim _{T \rightarrow \infty}\left\|\tilde{v}_{z}(\cdot, T)\right\|_{L^{2}\left(\Omega_{i}\right)}=0$, e satisfaz

$$
\int_{0}^{\infty} \int_{\Omega_{i}} \nabla \tilde{v}(x, z) \nabla \tilde{\varphi}(x, z) d x d z=\int_{\Omega_{i}} u^{p}(x) \tilde{\varphi}(x, 0) d x
$$

para qualquer $\tilde{\varphi} \in H_{\text {per }}^{1}\left(\mathbb{R}_{+}^{2}\right)$, par quase sempre na variável $x$,

$$
\int_{\Omega_{i}} \tilde{\varphi}(x, z) d x=0
$$

para qualquer $z \geq 0$ e $\lim _{T \rightarrow \infty}\|\tilde{\varphi}(\cdot, T)\|_{L^{2}\left(\Omega_{i}\right)}=\lim _{T \rightarrow \infty}\left\|\tilde{\varphi}_{z}(\cdot, T)\right\|_{L^{2}\left(\Omega_{i}\right)}=0$ 
Como vimos no início deste capítulo, exigiremos uma condição da solução $u$, de tal forma que $\left(u^{p}\right)_{\Omega_{i}}=0$. No caso do domínio $\Omega_{i}$, a condição será

$$
\tilde{v}(x+\pi, 0)=-\tilde{v}(x, 0)
$$

quase sempre para $x \in \mathbb{R}$.

Usando a paridade e periodicidade da função $\tilde{v}$ na variável $x$, segue que

$$
\begin{aligned}
\int_{0}^{\pi} \tilde{v}^{p}(x, 0) d x & =\int_{0}^{\pi}(-\tilde{v}(x+\pi, 0))^{p} d x \\
& =-\int_{0}^{\pi} \tilde{v}(x+\pi, 0)^{p} d x \\
& =-\int_{0}^{\pi} \tilde{v}(x, 0)^{p} d x,
\end{aligned}
$$

$\log 0$

$$
\int_{0}^{\pi} \tilde{v}(x, 0)^{p} d x=0
$$

\subsubsection{Solução fraca no quadrado}

Inicialmente, vejamos uma motivação para definição de solução fraca de (5.1).

Seja $u \in D\left(A_{\frac{1}{2}}\right)$, logo, para qualquer $(x, y) \in \Omega_{q}$,

$$
B_{\frac{1}{2}} u(x, y)=A_{\frac{1}{2}} u(x, y)=-\tilde{v}_{z}(x, y, 0)
$$

onde $\tilde{v}$ é solução clássica de

$$
\left\{\begin{array}{lllll}
\Delta \tilde{v}(x, y, z) & = & 0 & \text { em } & \mathbb{R}_{+}^{3} \\
\tilde{v}(x, y, 0) & = & \tilde{u}(x, y) & \text { em } & \mathbb{R}^{2} \\
\lim _{z \rightarrow \infty}\|\tilde{v}(\cdot, \cdot, z)\|_{L^{2}\left(\Omega_{q}\right)} & = & 0 & \\
\lim _{z \rightarrow \infty}\left\|\tilde{v}_{z}(\cdot, \cdot, z)\right\|_{L^{2}\left(\Omega_{q}\right)} & = & 0 & \\
(\tilde{v}(\cdot, \cdot, z))_{\Omega_{q}} & = & 0 & \forall z \geq 0
\end{array},\right.
$$

tal que $\tilde{u}$ é a extensão par, $2 \pi$-periódica nas variáveis $x$ e $y$ de $u$ em $\mathbb{R}^{2}$.

Considere $\tilde{\varphi} \in H_{p e r}^{1}\left(\mathbb{R}_{+}^{3}\right)$, par quase sempre nas variáveis $x$ e $y$ tal que $\tilde{\varphi}(x, y, 0)=\varphi(x, y)$,

$$
\int_{\Omega_{q}} \tilde{\varphi}(x, y, z) d x d y=0
$$

para qualquer $z \geq 0$ e $\lim _{z \rightarrow \infty}\|\tilde{\varphi}(\cdot, \cdot, z)\|_{L^{2}\left(\Omega_{q}\right)}=\lim _{z \rightarrow \infty}\left\|\tilde{\varphi}_{z}(\cdot, \cdot, z)\right\|_{L^{2}\left(\Omega_{q}\right)}=0$.

Logo,

$$
\int_{\Omega_{q}} u^{p}(x, y) \varphi(x, y) d x d y=\int_{\Omega_{q}} B_{\frac{1}{2}} u(x, y) \varphi(x, y) d x d y=-\int_{\Omega_{q}} \tilde{v}_{z}(x, y, 0) \varphi(x, y) d x d y .
$$

Como $\tilde{v}$ é solução clássica de (5.7), usando o Método de Integração por Partes, temos para qualquer 
$T>0$ que

$$
\begin{aligned}
\int_{0}^{T} \int_{\Omega_{q}} \nabla \tilde{v}(x, y, z) \nabla \tilde{\varphi}(x, y, z) d x d y d z= & -\int_{\Omega_{q}} \tilde{v}_{z}(x, y, 0) \tilde{\varphi}(x, y, 0) d x d y \\
& +\int_{\Omega_{q}} \tilde{v}_{z}(x, y, T) \tilde{\varphi}(x, y, T) d x d y
\end{aligned}
$$

Fazendo $T \rightarrow \infty$ temos

$$
\begin{aligned}
\int_{0}^{\infty} \int_{\Omega_{q}} \nabla \tilde{v}(x, y, z) \nabla \tilde{\varphi}(x, y, z) d x d y d z= & -\int_{\Omega_{q}} \tilde{v}_{z}(x, y, 0) \tilde{\varphi}(x, y, 0) d x d y \\
& +\lim _{T \rightarrow \infty} \int_{\Omega_{q}} \tilde{v}_{z}(x, y, T) \tilde{\varphi}(x, y, T) d x d y
\end{aligned}
$$

Usando a Desigualdade de Hölder temos

$$
\begin{aligned}
\left|\int_{\Omega_{q}} \tilde{v}_{z}(x, y, T) \tilde{\varphi}(x, y, T) d x d y\right| & \leq\left\|\tilde{v}_{z}(\cdot, \cdot, T)\right\|_{L^{2}\left(\Omega_{q}\right)}\|\tilde{\varphi}(\cdot, \cdot, T)\|_{L^{2}\left(\Omega_{q}\right)} \\
& \Longrightarrow \lim _{T \rightarrow \infty} \int_{\Omega_{q}} \tilde{v}_{z}(x, y, T) \tilde{\varphi}(x, y, T) d x d y=0
\end{aligned}
$$

Portanto, de (5.8) e (5.9) segue que

$$
\int_{0}^{\infty} \int_{\Omega_{q}} \nabla \tilde{v}(x, y, z) \nabla \tilde{\varphi}(x, y, z) d x d y d z=\int_{\Omega_{q}} \tilde{u}^{p}(x, y) \tilde{\varphi}(x, y, 0) d x d y
$$

para qualquer $\tilde{\varphi} \in H_{p e r}^{1}\left(\mathbb{R}_{+}^{3}\right)$, par quase sempre nas variáveis $x$ e $y$,

$$
\int_{\Omega_{q}} \tilde{\varphi}(x, y, z) d x d y=0
$$

para qualquer $z \geq 0$ tal que $\lim _{T \rightarrow \infty}\left\|\tilde{\varphi}_{z}(\cdot, \cdot, T)\right\|_{L^{2}\left(\Omega_{q}\right)}=\lim _{T \rightarrow \infty}\|\tilde{\varphi}(\cdot, \cdot, T)\|_{L^{2}\left(\Omega_{q}\right)}=0$.

Logo, temos a seguinte definição de solução fraca de (5.1) para $\Omega_{q}$.

Definição 18. Dizemos que u é solução fraca de (5.1) se $u=\left.(\tilde{v}(\cdot, \cdot, 0))\right|_{\Omega_{q}}$, onde $\tilde{v} \in H_{\text {per }}^{1}\left(\mathbb{R}_{+}^{3}\right)$, é par quase sempre nas variáveis $x$ e $y$,

$$
\int_{\Omega_{q}} \tilde{v}(x, y, z) d x d y=0
$$

para qualquer $z \geq 0, \lim _{T \rightarrow \infty}\|\tilde{v}(\cdot, \cdot, T)\|_{L^{2}\left(\Omega_{q}\right)}=\lim _{T \rightarrow \infty}\left\|\tilde{v}_{z}(\cdot, \cdot, T)\right\|_{L^{2}\left(\Omega_{q}\right)}=0$, e satisfaz

$$
\int_{0}^{\infty} \int_{\Omega_{q}} \nabla \tilde{v}(x, y, z) \nabla \tilde{\varphi}(x, y, z) d x d y d z=\int_{\Omega_{q}} u^{p}(x, y) \tilde{\varphi}(x, y, 0) d x d y
$$

para qualquer $\tilde{\varphi} \in H_{\text {per }}^{1}\left(\mathbb{R}_{+}^{3}\right)$, par quase sempre nas variáveis $x$ e $y$,

$$
\int_{\Omega_{q}} \tilde{\varphi}(x, y, z) d x d y=0
$$


para qualquer $z \geq 0$ e $\lim _{T \rightarrow \infty}\|\tilde{\varphi}(\cdot, \cdot, T)\|_{L^{2}\left(\Omega_{q}\right)}=\lim _{T \rightarrow \infty}\left\|\tilde{\varphi}_{z}(\cdot, \cdot, T)\right\|_{L^{2}\left(\Omega_{q}\right)}=0$.

Analogamente ao domínio $\Omega_{i}$, procuraremos soluções de (5.1) satisfazendo a seguinte condição

$$
\tilde{v}(x+\pi, y, 0)=-\tilde{v}(x, y, 0)
$$

quase sempre para $(x, y) \in \mathbb{R}^{2}$.

Com isso, pela paridade e periodicidade da função $\tilde{v}$ na variável $x$, temos que

$$
\begin{aligned}
\int_{0}^{\pi} \int_{0}^{\pi} \tilde{v}^{p}(x, y, 0) d x d y & =\int_{0}^{\pi} \int_{0}^{\pi}(-\tilde{v}(x+\pi, y, 0))^{p} d x d y \\
& =-\int_{0}^{\pi} \int_{0}^{\pi} \tilde{v}(x+\pi, y, 0)^{p} d x d y \\
& =-\int_{0}^{\pi} \int_{0}^{\pi} \tilde{v}(x, y, 0)^{p} d x d y
\end{aligned}
$$

$\log 0$

$$
\int_{0}^{\pi} \int_{0}^{\pi} \tilde{v}(x, y, 0)^{p} d x=0 .
$$

Observe que a condição anterior, poderia ser na variável y, ou seja,

$$
\tilde{v}(x, y+\pi, 0)=-\tilde{v}(x, y, 0)
$$

quase sempre para $(x, y) \in \mathbb{R}^{2}$.

\subsubsection{Existência de Solução Fraca}

Nosso objetivo é aplicar o Teorema do Multiplicador de Lagrange em espaços lineares topológicos, e assim obter a existência de solução fraca não nula do problema não linear (5.1). Para isto, vamos inicialmente estudar a existência do mínimo de um funcional apropriado.

Sejam as seguintes condições:

1. Se o domínio for $\Omega_{i}$

(a) $\tilde{v}(-x, z)=\tilde{v}(x, z)$, quase sempre em $x \in \mathbb{R}$, para qualquer $z \geq 0$,

(b) $\tilde{v}(x+\pi, 0)=-\tilde{v}(x, 0)$, quase sempre em $x \in \mathbb{R}$,

(c) $(\tilde{v}(\cdot, z))_{\Omega_{i}}=0$, para qualquer $z \geq 0$.

2. Se o domínio for $\Omega_{q}$

(a) $\tilde{v}(-x, y, z)=\tilde{v}(x,-y, z)=\tilde{v}(x, y, z)$, quase sempre em $(x, y) \in \mathbb{R}^{2}$, para qualquer $z \geq 0$,

(b) $\tilde{v}(x+\pi, y, 0)=-\tilde{v}(x, y, 0)$, quase sempre em $(x, y) \in \mathbb{R}^{2}$,

(c) $(\tilde{v}(\cdot, \cdot, z))_{\Omega_{q}}=0$, para qualquer $z \geq 0$. 
Considere os conjuntos, para cada tipo de domínio $\Omega$.

$$
\begin{aligned}
& H_{i}=\left\{\tilde{v} \in H_{p e r}^{1}\left(\mathbb{R}_{+}^{2}\right) \mid \tilde{v} \text { satisfaz } 1 \text { e } \lim _{T \rightarrow \infty}\|\tilde{v}(\cdot, T)\|_{L^{2}\left(\Omega_{i}\right)}=\lim _{T \rightarrow \infty}\left\|\tilde{v}_{z}(\cdot, T)\right\|_{L^{2}\left(\Omega_{i}\right)}=0\right\}, \\
& H_{q}=\left\{\tilde{v} \in H_{p e r}^{1}\left(\mathbb{R}_{+}^{3}\right) \mid \tilde{v} \text { satisfaz } 2 \text { e } \lim _{T \rightarrow \infty}\|\tilde{v}(\cdot, \cdot, T)\|_{L^{2}\left(\Omega_{q}\right)}=\lim _{T \rightarrow \infty}\left\|\tilde{v}_{z}(\cdot, \cdot, T)\right\|_{L^{2}\left(\Omega_{q}\right)}=0\right\} .
\end{aligned}
$$

Lema 12. Existem $\tilde{v}_{1}, \tilde{v}_{2}$ não nulas tais que $\tilde{v}_{1} \in H_{i}$ e $\tilde{v}_{2} \in H_{q}$. E ainda,

$$
\left(H_{i},\|\cdot\|_{H_{p e r}^{1}\left(\mathbb{R}_{+}^{2}\right)}\right) \quad e \quad\left(H_{q},\|\cdot\|_{H_{p e r}^{1}\left(\mathbb{R}_{+}^{3}\right)}\right),
$$

são Espaços de Hilbert.

Demonstração. Considere

$$
\tilde{v}_{1}(x, z)=e^{-z} \cos (x) \text { e } \quad \tilde{v}_{2}(x, y, z)=e^{-z} \cos (x) \cos (y) .
$$

Facilmente podemos verificar que $\tilde{v}_{1}$ e $\tilde{v}_{2}$ são funções não nulas e estão em $H_{i}$ e $H_{q}$, respectivamente.

Agora o objetivo é mostrar que $H_{i}$ e $H_{q}$ são fechados em $H_{p e r}^{1}\left(\mathbb{R}_{+}^{2}\right)$ e $H_{p e r}^{1}\left(\mathbb{R}_{+}^{3}\right)$, respectivamente. Mostraremos para $H_{q}$, pois o caso $H_{i}$ é análogo.

Seja $\left\{\tilde{v}_{n}\right\}_{n \in \mathbb{N}} \subset H_{q}$ tal que

$$
\left\|\tilde{v}_{n}-\tilde{v}\right\|_{H_{p e r}^{1}\left(\mathbb{R}_{+}^{3}\right)} \stackrel{n \rightarrow \infty}{\longrightarrow} 0
$$

$\operatorname{com} \tilde{v} \in H_{p e r}^{1}\left(\mathbb{R}_{+}^{3}\right)$.

1. Mostremos que

$$
\tilde{v}(-x, y, z)=\tilde{v}(x, y, z) \quad \text { e } \tilde{v}(x,-y, z)=\tilde{v}(x, y, z)
$$

para todo $(x, y) \in \mathbb{R}^{2}$, exceto em um conjunto de medida nula e para qualquer $z \geq 0$.

Seja

$$
\tilde{s}(x, y, z)=\tilde{v}(-x, y, z)
$$

quase sempre em $(x, y) \in \mathbb{R}^{2}$, para qualquer $z \geq 0$. Logo,

$$
\begin{aligned}
\int_{-\pi}^{\pi} \int_{-\pi}^{\pi}|\tilde{v}(x, y, z)-\tilde{s}(x, y, z)| d x d y & \leq \int_{-\pi}^{\pi} \int_{-\pi}^{\pi}\left|\tilde{v}(x, y, z)-\tilde{v}_{n}(x, y, z)\right| d x d y \\
& +\int_{-\pi}^{\pi} \int_{-\pi}^{\pi}\left|\tilde{v}_{n}(x, y, z)-\tilde{s}(x, y, z)\right| d x d y \\
& \leq C\left\|\tilde{v}(\cdot, \cdot, z)-\tilde{v}_{n}(\cdot, \cdot, z)\right\|_{L_{p e r}^{2}\left(\mathbb{R}^{2}\right)} \\
& \leq C\left\|\tilde{v}(\cdot, \cdot, z)-\tilde{v}_{n}(\cdot, \cdot, z)\right\|_{H_{p e r} \frac{1}{2}\left(\mathbb{R}^{2}\right)}
\end{aligned}
$$

Aplicando o Teorema do Traço, temos que

$$
\int_{-\pi}^{\pi} \int_{-\pi}^{\pi}|\tilde{v}(x, y, z)-\tilde{s}(x, y, z)| d x \leq C\left\|\tilde{v}-\tilde{v}_{n}\right\|_{H_{p e r}^{1}\left(\mathbb{R}_{+}^{3}\right)} \stackrel{n \rightarrow \infty}{\longrightarrow} 0,
$$


para qualquer $z \geq 0$.

Logo,

$$
\tilde{v}(x, y, z)=\tilde{s}(x, y, z)=\tilde{v}(-x, y, z)
$$

quase sempre em $(x, y) \in(-\pi, \pi) \times(-\pi, \pi)$, para qualquer $z \geq 0$, logo pela periodicidade, temos que (5.10) vale quase sempre em $\mathbb{R}^{2}$, para qualquer $z \geq 0$.

Analogamente, verifica-se que

$$
\tilde{v}(x,-y, z)=\tilde{v}(x, y, z)
$$

quase sempre em $\mathbb{R}^{2}$, para qualquer $z \geq 0$.

2. Vejamos que

$$
\int_{\Omega_{q}} \tilde{v}(x, y, z) d x d y=0
$$

para qualquer $z \geq 0$.

De fato, seja $z \geq 0$, então pela Desigualdade de Hölder e o Teorema do Traço, segue que

$$
\begin{aligned}
\left|\int_{\Omega_{q}} \tilde{v}(x, y, z) d x d y\right| & \leq \int_{\Omega_{q}}\left|\tilde{v}(x, y, z)-\tilde{v}_{n}(x, y, z)\right| d x d y \\
& =\frac{1}{4} \int_{Q^{2}}\left|\tilde{v}(x, y, z)-\tilde{v}_{n}(x, y, z)\right| d x d y \\
& \leq C\left\|\tilde{v}-\tilde{v}_{n}\right\|_{H_{p e r}^{1}\left(\mathbb{R}_{+}^{3}\right)} \stackrel{n \rightarrow \infty}{\longrightarrow},
\end{aligned}
$$

então

$$
\int_{\Omega_{q}} \tilde{v}(x, y, z) d x d y=0
$$

para qualquer $z \geq 0$.

3. Temos ainda, que

$$
\tilde{v}(x+\pi, y, 0)=-\tilde{v}(x, y, 0),
$$

quase sempre em $(x, y) \in \mathbb{R}^{2}$.

De fato,

$$
\begin{aligned}
\int_{0}^{\pi} \int_{0}^{\pi}|\tilde{v}(x+\pi, y, 0)+\tilde{v}(x, y, 0)| d x d y & \leq \int_{0}^{\pi} \int_{0}^{\pi}\left|\tilde{v}(x+\pi, y, 0)-\tilde{v}_{n}(x+\pi, y, 0)\right| d x d y \\
& +\int_{0}^{\pi} \int_{0}^{\pi}\left|\tilde{v}_{n}(x+\pi, y, 0)+\tilde{v}(x, y, 0)\right| d x d y \\
& =\int_{0}^{\pi} \int_{\pi}^{2 \pi}\left|\tilde{v}(t, y, 0)-\tilde{v}_{n}(t, y, 0)\right| d t d y \\
& +\int_{0}^{\pi} \int_{0}^{\pi}\left|-\tilde{v}_{n}(x, y, 0)+\tilde{v}(x, y, 0)\right| d x d y \\
& =\int_{0}^{\pi} \int_{-\pi}^{0}\left|\tilde{v}(t, y, 0)-\tilde{v}_{n}(t, y, 0)\right| d t d y \\
& +\int_{0}^{\pi} \int_{0}^{\pi}\left|-\tilde{v}_{n}(x, y, 0)+\tilde{v}(x, y, 0)\right| d x d y
\end{aligned}
$$


$\log 0$

$$
\begin{aligned}
\int_{0}^{\pi} \int_{0}^{\pi}|\tilde{v}(x+\pi, y, 0)+\tilde{v}(x, y, 0)| d x d y & \leq \int_{0}^{\pi} \int_{-\pi}^{\pi}\left|\tilde{v}(x, y, 0)-\tilde{v}_{n}(x, y, 0)\right| d x d y \\
& \leq \int_{Q^{2}}\left|\tilde{v}(x, y, 0)-\tilde{v}_{n}(x, y, 0)\right| d x d y
\end{aligned}
$$

Pela Desigualdade de Hölder e o Teorema do Traço, segue que

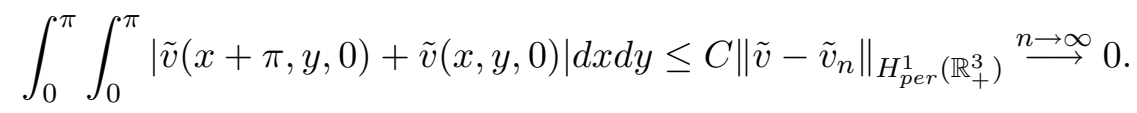

Portanto,

$$
\tilde{v}(x+\pi, y, 0)=-\tilde{v}(x, y, 0),
$$

quase sempre em $(x, y) \in(0, \pi) \times(0, \pi)$, onde pela paridade e periodicidade de $\tilde{v}$, segue que a igualdade vale quase sempre em $\mathbb{R}^{2}$.

4 .

$$
\lim _{T \rightarrow \infty}\|\tilde{v}(\cdot, \cdot, T)\|_{L^{2}\left(\Omega_{q}\right)}=\lim _{T \rightarrow \infty}\left\|\tilde{v}_{z}(\cdot, \cdot, T)\right\|_{L^{2}\left(\Omega_{q}\right)}=0 .
$$

De fato, suponha que $\lim _{T \rightarrow \infty}\|\tilde{v}(\cdot, \cdot, T)\|_{L^{2}\left(\Omega_{q}\right)} \neq 0$, logo,

$$
\text { ou } \lim _{T \rightarrow \infty}\|\tilde{v}(\cdot, \cdot, T)\|_{L^{2}\left(\Omega_{q}\right)}=\infty \text { ou } \lim _{T \rightarrow \infty}\|\tilde{v}(\cdot, \cdot, T)\|_{L^{2}\left(\Omega_{q}\right)}=P, \quad \text { com } P \neq 0
$$

Se $\lim _{T \rightarrow \infty}\|\tilde{v}(\cdot, \cdot, T)\|_{L^{2}\left(\Omega_{q}\right)}=\infty$, então $\forall \epsilon>0, \exists \delta>0$ tal que

$$
T>\delta \Longrightarrow\|\tilde{v}(\cdot, \cdot, T)\|_{L^{2}\left(\Omega_{q}\right)} \geq \epsilon
$$

Logo,

$$
\begin{aligned}
\int_{0}^{\infty} \int_{\Omega_{q}}|\tilde{v}(x, y, z)|^{2} d x d y d z & =\int_{0}^{\delta} \int_{\Omega_{q}}|\tilde{v}(x, y, z)|^{2} d x d y d z \\
& +\int_{\delta}^{\infty} \int_{\Omega_{q}}|\tilde{v}(x, y, z)|^{2} d x d y d z \\
& \geq \int_{\delta}^{\infty}\|\tilde{v}(\cdot, \cdot, z)\|_{L^{2}\left(\Omega_{q}\right)}^{2} d z \\
& =\infty
\end{aligned}
$$

o que é absurdo, pois $\tilde{v} \in H_{\text {per }}^{1}\left(\mathbb{R}_{+}^{3}\right)$.

Se $\lim _{T \rightarrow \infty}\|\tilde{v}(\cdot, \cdot, T)\|_{L^{2}(\Omega)}=P$, com $P \neq 0$, então $\forall \epsilon>0, \exists \delta>0$ tal que

$$
T>\delta \Longrightarrow\|\tilde{v}(\cdot, \cdot, T)\|_{L^{2}(\Omega)} \geq P-\epsilon,
$$

onde podemos tomar $\epsilon<P$. 
Assim, analogamente ao que fizemos anteriormente, temos

$$
\begin{aligned}
\left.\int_{0}^{\infty} \int_{\Omega_{q}} \tilde{v}(x, y, z)\right|^{2} d x d y d z & \geq \int_{\delta}^{\infty}\|\tilde{v}(\cdot, \cdot, z)\|_{L^{2}(\Omega)}^{2} d z \\
& \geq(P-\epsilon)^{2} \int_{\delta}^{\infty} d z \\
& =\infty
\end{aligned}
$$

o que é absurdo, pois $\tilde{v} \in H_{p e r}^{1}\left(\mathbb{R}_{+}^{3}\right)$. Portanto, $\lim _{T \rightarrow \infty}\|\tilde{v}(\cdot, \cdot, T)\|_{L^{2}\left(\Omega_{q}\right)}=0$.

Analogamente, podemos supor que $\lim _{T \rightarrow \infty}\left\|\tilde{v}_{z}(\cdot, \cdot, T)\right\|_{L^{2}\left(\Omega_{q}\right)} \neq 0$ e pelas mesmas contas feitas nos dois casos anteriores, obtemos que

$$
\int_{0}^{\infty} \int_{\Omega_{q}}|\nabla \tilde{v}(x, y, z)|^{2} \geq \infty
$$

que é absurdo, pois $\tilde{v} \in H_{p e r}^{1}\left(\mathbb{R}_{+}^{3}\right)$. Portanto, $\lim _{T \rightarrow \infty}\left\|\tilde{v}_{z}(\cdot, \cdot, T)\right\|_{L^{2}\left(\Omega_{q}\right)}=0$.

Finalmente, concluímos que $\tilde{v} \in H_{q}$ e assim $\left(H_{q},\|\cdot\|_{H_{p e r}^{1}\left(\mathbb{R}_{+}^{3}\right)}\right)$ é um espaço de Hilbert.

Vejamos agora, equivalências para as normas de $H_{i}$ e $H_{q}$. Estas equivalências serão fundamentais para mostrarmos a existência do mínimo de um funcional apropriado.

Lema 13. Considere as seguintes normas

$$
\|\tilde{v}\|_{i}=\left(\int_{0}^{\infty} \int_{\Omega_{i}}|\nabla \tilde{v}(x, z)|^{2} d x d z\right)^{\frac{1}{2}}
$$

$e$

$$
\|\tilde{v}\|_{q}=\left(\int_{0}^{\infty} \int_{\Omega_{q}}|\nabla \tilde{v}(x, y, z)|^{2} d x d y d z\right)^{\frac{1}{2}},
$$

em $H_{i}$ e $H_{q}$, respectivamente. Então, as normas $\|\cdot\|_{i}$ e $\|\cdot\|_{H_{p e r}^{1}\left(\mathbb{R}_{+}^{2}\right)}$ são equivalentes em $H_{i}$, bem como as normas $\|\cdot\|_{q}$ e $\|\cdot\|_{H_{p e r}^{1}\left(\mathbb{R}_{+}^{3}\right)}$ são equivalentes em $H_{q}$.

Demonstração. Seja $\tilde{v} \in H_{q}$, é óbvio que

$$
\|\tilde{v}\|_{q} \leq \frac{1}{2}\|\tilde{v}\|_{H_{p e r}^{1}\left(\mathbb{R}_{+}^{3}\right)} .
$$

Por outro lado, pela Proposição 4., existe $C>0$ tal que para qualquer $\tilde{v} \in H_{q}$

$$
\begin{aligned}
\int_{0}^{\infty} \int_{\Omega_{q}}|\tilde{v}(x, y, z)|^{2} d x d y d z & \leq C\left(\int_{0}^{\infty} \int_{\Omega_{q}} \tilde{v}(x, y, z) d x d y d z\right)^{2} \\
& +C \int_{0}^{\infty} \int_{\Omega_{q}}|\nabla \tilde{v}(x, y, z)|^{2} d x d y d z
\end{aligned}
$$

Mas, sabemos que

$$
\int_{\Omega_{q}} \tilde{v}(x, y, z) d x d y=0
$$


para qualquer $z \geq 0$, logo

$$
\int_{0}^{\infty} \int_{\Omega_{q}} \tilde{v}(x, y, z) d x d y d z=0
$$

Então,

$$
\begin{aligned}
\int_{0}^{\infty} \int_{\Omega_{q}}|\tilde{v}(x, y, z)|^{2} d x d y d z & \leq C \int_{0}^{\infty} \int_{\Omega_{q}}|\nabla \tilde{v}(x, y, z)|^{2} d x d y d z \\
& =C\|\tilde{v}\|_{q}^{2} \\
& \Longrightarrow\|\tilde{v}\|_{H_{p e r}^{1}\left(\mathbb{R}_{+}^{3}\right)} \leq[2(1+C)]^{\frac{1}{2}}\|\tilde{v}\|_{q} .
\end{aligned}
$$

Portanto, as normas $\|\cdot\|_{H_{p e r}^{1}\left(\mathbb{R}_{+}^{3}\right)}$ e $\|\cdot\|_{q}$ são equivalentes em $H_{q}$. Analogamente, mostramos que as normas $\|\cdot\|_{i} \mathrm{e}\|\cdot\|_{H_{p e r}^{1}\left(\mathbb{R}_{+}^{2}\right)}$ são equivalentes em $H_{i}$.

Observação 6. Daqui pra frente, omitiremos $(x, z) e(x, y, z)$ das integrais, para não carregar a notação.

Considere as aplicações $I_{i}: H_{i} \longrightarrow \mathbb{R}$ e $I_{q}: H_{q} \longrightarrow \mathbb{R}$, tais que

$$
I_{i}(\tilde{r})=\frac{1}{2} \int_{0}^{\infty} \int_{\Omega_{i}}|\nabla \tilde{r}|^{2} d x d z \quad \text { e } \quad I_{q}(\tilde{v})=\frac{1}{2} \int_{0}^{\infty} \int_{\Omega_{q}}|\nabla \tilde{v}|^{2} d x d y d z
$$

para qualquer $\tilde{r} \in H_{i}, \tilde{v} \in H_{q}$.

Para algum $a>0$ fixo, considere os seguintes conjuntos

$$
\begin{aligned}
H_{i, a} & =\left\{\tilde{v} \in H_{i} \mid \int_{\Omega_{i}}(\tilde{v}(x, 0))^{p+1} d x=a\right\}, \\
H_{q, a} & =\left\{\tilde{v} \in H_{q} \mid \int_{\Omega_{q}}(\tilde{v}(x, y, 0))^{p+1} d x d y=a\right\} .
\end{aligned}
$$

Proposição 11. Existe $\tilde{v} \in H_{q, a}$ tal que

$$
I_{q}(\tilde{v})=\min _{\tilde{w} \in H_{q, a}} I_{q}(\tilde{w}) .
$$

Demonstração. Seja

$$
m=\inf \left\{I_{q}(\tilde{v}) \mid \tilde{v} \in H_{q, a}\right\} .
$$

Temos, que o conjunto $\left\{I_{q}(\tilde{v}) \mid \tilde{v} \in H_{q, a}\right\}$ é não vazio, pois dada $\tilde{v} \in H_{q}$ não nula, podemos considerar

$$
\tilde{w}=\frac{a^{\frac{1}{p+1}}}{\left(\int_{\Omega_{q}}(\tilde{v}(x, y, 0))^{p+1} d x d y\right)^{\frac{1}{p+1}}} \tilde{v} \in H_{q, a},
$$

e como $I_{q}(\tilde{w})<\infty$ segue o resultado.

Observe também que $0 \leq m<\infty$, pois se $m=\infty$ teríamos para qualquer $\tilde{v} \in H_{q, a}$ que

$$
\infty=m \leq I_{q}(\tilde{v})<\infty
$$


o que é absurdo.

Por definição de ínfimo, podemos tomar uma sequência minimizante, isto é, $\left\{\tilde{v}_{j}\right\}_{j \in \mathbb{N}} \subset H_{q, a}$ tal que

$$
\lim _{j \rightarrow \infty} I_{q}\left(\tilde{v}_{j}\right)=m
$$

Como $\left\{I_{q}\left(\tilde{v}_{j}\right)\right\}_{j \in \mathbb{N}} \subset \mathbb{R}$, temos que existe $M>0$ tal que

$$
\left|I_{q}\left(\tilde{v}_{j}\right)\right| \leq M
$$

para qualquer $j \in \mathbb{N}$.

Logo,

$$
\left\|\tilde{v}_{j}\right\|_{q}^{2}=\int_{0}^{\infty} \int_{\Omega_{q}}\left|\nabla \tilde{v}_{j}\right|^{2} d x d y d z=2 I_{q}\left(\tilde{v}_{j}\right) \leq 2 M
$$

assim a sequência $\left\{\tilde{v}_{j}\right\}_{j \in \mathbb{N}}$ é limitada em $H_{q}$.

Então, usando imersão compacta existe uma subsequência $\left\{\tilde{v}_{j k}\right\}_{k \in \mathbb{N}}$ e $\tilde{v} \in H_{q}$ tal que

$$
\left\{\begin{array}{cllll}
\tilde{v}_{j k} & \rightarrow & \tilde{v} & \text { em } & H_{q} \\
\tilde{v}_{j k}(\cdot, \cdot, 0) & \rightarrow & \tilde{v}(\cdot, \cdot, 0) & \text { em } & L^{p+1}\left(Q^{2}\right)
\end{array}\right.
$$

onde os símbolos $\rightarrow \mathrm{e} \rightarrow$ denotam, respectivamente, convergência fraca e forte.

Observe que

$$
\left|\int_{\Omega_{q}}(\tilde{v}(x, y, 0))^{p+1} d x d y-a\right|=\left|\int_{\Omega_{q}}(\tilde{v}(x, y, 0))^{p+1}-\left(\tilde{v}_{j k}(x, y, 0)\right)^{p+1} d x\right| .
$$

Pelo Teorema do Valor Médio ( [13]), aplicado a função,

$$
\begin{aligned}
& G:\left[\tilde{v}_{j k}(x, y, 0), \tilde{v}(x, y, 0)\right] \quad \longrightarrow \quad \mathbb{R} \\
& t \quad \longmapsto t^{p+1}
\end{aligned}
$$

para cada $(x, y) \in \Omega_{q}$, temos que existe $\tilde{w}_{j k}(x, y, 0) \in\left(\tilde{v}_{j k}(x, y, 0), \tilde{v}(x, y, 0)\right)$ tal que

$$
(\tilde{v}(x, y, 0))^{p+1}-\left(\tilde{v}_{j k}(x, y, 0)\right)^{p+1}=(p+1)\left(\tilde{w}_{j k}(x, y, 0)\right)^{p}\left(\tilde{v}(x, y, 0)-\tilde{v}_{j k}(x, y, 0)\right) .
$$

Logo,

$$
\left|\int_{\Omega_{q}}(\tilde{v}(x, y, 0))^{p+1} d x d y-a\right| \leq(p+1) \int_{\Omega_{q}}\left|\tilde{w}_{j k}(x, y, 0)\right|^{p}\left|\tilde{v}(x, y, 0)-\tilde{v}_{j k}(x, y, 0)\right| d x d y
$$

Temos que $\tilde{v}(\cdot, \cdot, 0), \tilde{v}_{j k}(\cdot, \cdot, 0) \in L^{p+1}\left(Q^{2}\right), \log 0$

$$
w_{j k}(\cdot, \cdot, 0) \in L^{p+1}\left(Q^{2}\right) \Longrightarrow w_{j k}^{p}(\cdot, \cdot, 0) \in L^{\frac{p+1}{p}}\left(Q^{2}\right)
$$

para qualquer $j, k \in \mathbb{N}$, onde $\frac{p+1}{p}$ é o dual de $p+1$. 
Assim, pela Desigualdade de Hölder e pelo Teorema do Traço, segue que

$$
\begin{aligned}
\left|\int_{\Omega_{q}}(\tilde{v}(x, y, 0))^{p+1} d x d y-a\right| & \leq(p+1)\left\|\tilde{w}_{j k}(\cdot, \cdot, 0)\right\|_{L^{p+1}\left(Q^{2}\right)}^{p}\left\|\tilde{v}(\cdot, \cdot, 0)-\tilde{v}_{j k}(\cdot, \cdot, 0)\right\|_{L^{p+1}\left(Q^{2}\right)} \\
& \leq C\left\|\tilde{w}_{j k}\right\|_{H_{p e r}^{1}\left(R_{+}^{3}\right)}^{p}\left\|\tilde{v}(\cdot, \cdot, 0)-\tilde{v}_{j k}(\cdot, \cdot, 0)\right\|_{L^{p+1}\left(Q^{2}\right)} \\
& =C\left(2 I\left(\tilde{w}_{j k}\right)\right)^{\frac{p}{2}}\left\|\tilde{v}(\cdot, \cdot, 0)-\tilde{v}_{j k}(\cdot, \cdot, 0)\right\|_{L^{p+1}\left(Q^{2}\right)} \\
& \leq C(2 M)^{\frac{p}{2}}\left\|\tilde{v}(\cdot, \cdot, 0)-\tilde{v}_{j k}(\cdot, \cdot, 0)\right\|_{L^{p+1}\left(Q^{2}\right)} \stackrel{k \rightarrow \infty}{\longrightarrow} 0 .
\end{aligned}
$$

Então,

$$
\int_{\Omega_{q}}(\tilde{v}(x, y, 0))^{p+1} d x d y=a,
$$

portanto $\tilde{v} \in H_{q, a}$ e assim temos que

$$
I_{q}(\tilde{v}) \geq \inf \left\{I_{q}(\tilde{w}) \mid \tilde{w} \in H_{q, a}\right\}=m .
$$

Por outro lado, a função $f: \mathbb{R}^{3} \longrightarrow \mathbb{R}$ tal que $f(a, b, c)=a^{2}+b^{2}+c^{2}$, para qualquer $(a, b, c) \in \mathbb{R}^{3}$, é uma função convexa, então pelo Teorema 13., vale que

$$
\frac{1}{2}\left|\nabla \tilde{v}_{j k}\right|^{2} \geq-\frac{1}{2}|\nabla \tilde{v}|^{2}+\nabla \tilde{v} \cdot \nabla \tilde{v}_{j k},
$$

ou seja,

$$
I_{q}\left(\tilde{v}_{j k}\right) \geq-I_{q}(\tilde{v})+\int_{0}^{\infty} \int_{\Omega_{q}} \nabla \tilde{v} \cdot \nabla \tilde{v}_{j k} d x d y d z
$$

Como $\tilde{v}_{j k} \rightarrow \tilde{v}$ em $H_{q}$, temos que $\nabla \tilde{v}_{j k} \rightarrow \nabla \tilde{v}$ em $L^{2}\left(\Omega_{q} \times(0, \infty) ; \mathbb{R}^{3}\right)$, logo

$$
\int_{0}^{\infty} \int_{\Omega_{q}} \nabla \tilde{v} \nabla \tilde{v}_{j k} d x d y d z \stackrel{k \rightarrow \infty}{\longrightarrow} \int_{0}^{\infty} \int_{\Omega_{q}}|\nabla \tilde{v}|^{2} d x d y d z
$$

Assim, fazendo $k \rightarrow \infty$ em (5.12), temos que

$$
m \geq I_{q}(\tilde{v})
$$

Portanto, $I_{q}(\tilde{v})=m$, isto é,

$$
I_{q}(\tilde{v})=\min _{\tilde{w} \in H_{q, a}} I_{q}(\tilde{w}) .
$$

Enunciaremos a seguir, o mesmo resultado anterior para o domínio $\Omega_{i}$, cuja demonstração é análoga ao caso $\Omega_{q}$, já que as imersões usadas continuam válidas.

Proposição 12. Existe $\tilde{v} \in H_{i, a}$ tal que

$$
I_{i}(\tilde{v})=\min _{\tilde{w} \in H_{i, a}} I_{i}(\tilde{w})
$$

Com a ajuda do Teorema do Multiplicador de Lagrange em espaços lineares topológicos, vamos 
mostrar a existência de solução fraca não nula do problema não linear (5.1).

Teorema 12. O problema não linear (5.1) admite solução fraca não nula, onde o domínio pode ser $\Omega_{i}$ ou $\Omega_{q}$.

Demonstração. Faremos a demonstração para o caso em que o domínio é $\Omega_{q}$. A demonstração para o domínio $\Omega_{i}$ é análoga.

Considere no Teorema 16. os espaços $E=G=U=H_{q}$, as funções

$$
\begin{aligned}
g: H_{q} & \longrightarrow \mathbb{R} \\
\tilde{w} & \longmapsto \frac{1}{p+1} \int_{\Omega_{q}}(\tilde{w}(x, y, 0))^{p+1} d x d y-\frac{a}{p+1},
\end{aligned}
$$

e

$$
\begin{aligned}
f=I_{q}: H_{q} & \longrightarrow \mathbb{R} \\
\tilde{w} & \longmapsto \frac{1}{2} \int_{0}^{\infty} \int_{\Omega_{q}}|\nabla \tilde{w}|^{2} d x d y d z .
\end{aligned}
$$

Tomemos $x_{0}=\tilde{v} \in H_{q, a}$, encontrado na Proposição 11. e

$$
M=\left\{\tilde{w} \in H_{q} \mid g(\tilde{w})=0\right\}=H_{q, a} .
$$

A demonstração consiste praticamente, em verificar as hipóteses do Teorema do Multiplicador de Lagrange em espaços lineares topológicos.

1. Pela Proposição 11., temos que $f(\tilde{v})$ é um mínimo de $f\left(H_{q, a}\right)$.

2. $f$ é fortemente $H_{q}$-diferenciável em $\tilde{v}$.

De fato, considere $D f(\tilde{v}): H_{q} \longrightarrow \mathbb{R}$ tal que para qualquer $\tilde{\varphi} \in H_{q}$ temos

$$
<D f(\tilde{v}), \tilde{\varphi}>=\int_{0}^{\infty} \int_{\Omega_{q}} \nabla \tilde{v} \nabla \tilde{\varphi} d x d y d z
$$

Logo,

$$
|f(\tilde{v}+\tilde{\varphi})-f(\tilde{v})-<D f(\tilde{v}), \tilde{\varphi}>|=\frac{1}{2}\|\tilde{\varphi}\|_{H_{q}}^{2} .
$$

Assim, dado $\epsilon>0$ tome $\delta \leq \sqrt{2 \epsilon}$, logo

$$
\|\tilde{\varphi}\|_{H_{q}} \leq \delta \Longrightarrow|f(\tilde{v}+\tilde{\varphi})-f(\tilde{v})-<D f(\tilde{v}), \tilde{\varphi}>| \leq \epsilon .
$$

Portanto, $f$ é Fréchet diferenciável em $\tilde{v}$ e assim pelo Lema 19., segue que $f$ é fortemente $H_{q^{-}}$ diferenciável em $\tilde{v}$.

3. $g$ é $H_{q}$-diferenciável em $\tilde{v}$.

De fato, seja $D g(\tilde{v}): H_{q} \longrightarrow \mathbb{R}$ tal que para qualquer $\tilde{\varphi} \in H_{q}$

$$
<D g(\tilde{v}), \tilde{\varphi}>=\int_{\Omega_{q}}(\tilde{v}(x, y, 0))^{p} \tilde{\varphi}(x, y, 0) d x d y
$$


Então,

$$
\begin{aligned}
\left|\frac{g(\tilde{v}+t \tilde{\varphi})-g(\tilde{v})}{t}-<D g(\tilde{v}), \tilde{\varphi}>\right| & =\frac{1}{|t|} \mid \frac{1}{p+1} \int_{\Omega_{q}}(\tilde{v}(x, y, 0)+t \tilde{\varphi}(x, y, 0))^{p+1} d x \\
& -\frac{1}{p+1} \int_{\Omega_{q}}(\tilde{v}(x, y, 0))^{p+1} d x d y \\
& -t \int_{\Omega_{q}}(\tilde{v}(x, y, 0))^{p} \tilde{\varphi}(x, y, 0) d x d y \mid .
\end{aligned}
$$

Sabemos que

$$
p+1=3+\frac{1}{s},
$$

onde $s>1$ ímpar. Então, a função $F: \mathbb{R} \longrightarrow \mathbb{R}$ tal que $F(s)=s^{p+1}$, é 3 -vezes diferenciável. Assim, pela Fórmula de Taylor Infinitesimal de [24], temos para cada $(x, y) \in \Omega_{q}$, que

$$
\begin{aligned}
\frac{1}{p+1} \int_{\Omega_{q}}(\tilde{v}(x, y, 0)+t \tilde{\varphi}(x, y, 0))^{p+1} d x d y & =\frac{1}{p+1} \int_{\Omega_{q}}(\tilde{v}(x, y, 0))^{p+1} d x d y \\
& +t \int_{\Omega_{q}}\left(\tilde{v}(x, y, 0)^{p}\right) \tilde{\varphi}(x, y, 0) d x d y \\
& +\frac{p t^{2}}{2 !} \int_{\Omega_{q}}(\tilde{v}(x, y, 0))^{p-1}(\tilde{\varphi}(x, y, 0))^{2} d x d y \\
& +\frac{p(p-1)}{3 !} \int_{\Omega_{q}}(\tilde{v}(x, y, 0))^{p-2}(\tilde{\varphi}(x, y, 0))^{3} d x d y \\
& +\frac{1}{p+1} \int_{\Omega_{q}} r_{3}(t \tilde{\varphi}(x, y, 0)) d x d y,
\end{aligned}
$$

onde $\lim _{t \rightarrow 0} \frac{r_{3}(t \tilde{\varphi}(x, y, 0))}{(t \tilde{\varphi}(x, y, 0))^{3}}=0$.

Então, em (5.13), segue que

$$
\begin{aligned}
\left|\frac{g(\tilde{v}+t \tilde{\varphi})-g(\tilde{v})}{t}-<D g(\tilde{v}), \tilde{\varphi}>\right| & \leq \frac{p|t|}{2 !} \int_{\Omega_{q}}|\tilde{v}(x, y, 0)|^{p-1}|\tilde{\varphi}(x, y, 0)|^{2} d x d y \\
& +\frac{p(p-1)|t|^{2}}{3 !} \int_{\Omega_{q}}|\tilde{v}(x, y, 0)|^{p-2}|\tilde{\varphi}(x, y, 0)|^{3} d x d y \\
& +\frac{1}{p+1} \int_{\Omega_{q}}\left|r_{3}(t \tilde{\varphi}(x, y, 0))\right| d x d y .
\end{aligned}
$$

Neste caso, usando as seguintes imersões

$$
H_{p e r}^{\frac{1}{2}}\left(\mathbb{R}^{2}\right) \hookrightarrow L^{4}\left(Q^{2}\right), \quad H_{p e r}^{\frac{1}{2}}\left(\mathbb{R}^{2}\right) \hookrightarrow L^{2(p-1)}\left(Q^{2}\right) \quad \text { e } \quad H_{p e r}^{\frac{1}{2}}\left(\mathbb{R}^{2}\right) \hookrightarrow L^{\frac{6(p-1)}{p}}\left(Q^{2}\right)
$$


e aplicando a Desigualdade de Hölder, temos que

$$
\begin{aligned}
\left|\frac{g(\tilde{v}+t \tilde{\varphi})-g(\tilde{v})}{t}-<D g(\tilde{v}), \tilde{\varphi}>\right| & \leq \frac{p|t|}{2 !}\|\tilde{v}(\cdot, \cdot, 0)\|_{L^{2(p-1)}\left(Q^{2}\right)}^{p-1}\|\tilde{\varphi}(\cdot, \cdot, 0)\|_{L^{4}\left(Q^{2}\right)}^{2} \\
& +\frac{p(p-1)|t|^{2}}{3 !}\|\tilde{v}(\cdot, \cdot, 0)\|_{L^{2(p-1)}\left(Q^{2}\right)}^{p-2}\|\tilde{\varphi}(\cdot, \cdot, 0)\|_{L^{\frac{6(p-1)}{p}}\left(Q^{2}\right)}^{\frac{1}{3}} \\
& +\frac{1}{p+1} \int_{\Omega_{q}}\left|r_{3}(t \tilde{\varphi}(x, y, 0))\right| d x d y .
\end{aligned}
$$

Por outro lado,

$$
\lim _{t \rightarrow 0} \frac{r_{3}(t \tilde{\varphi}(x, y, 0))}{(t \tilde{\varphi}(x, y, 0))^{3}}=0,
$$

ou seja, dado $\epsilon>0$, existe $\delta>0$ tal que

$$
|t|<\delta \Longrightarrow\left|r_{3}(t \tilde{\varphi}(x, y, 0))\right|<\epsilon|t|^{3}|\tilde{\varphi}(x, y, 0)|^{3},
$$

então

$$
\int_{\Omega_{q}}\left|r_{3}(t \tilde{\varphi}(x, y, 0))\right|<\epsilon \delta^{3}\|\tilde{\varphi}(\cdot, \cdot, 0)\|_{L^{3}\left(Q^{2}\right)}^{3}
$$

Assim, se $|t|<\delta$, segue que

$$
\begin{aligned}
\left|\frac{g(\tilde{v}+t \tilde{\varphi})-g(\tilde{v})}{t}-<D g(\tilde{v}), \tilde{\varphi}>\right| & \leq \frac{p \delta}{2 !}\|\tilde{v}(\cdot, \cdot, 0)\|_{L^{2(p-1)}\left(Q^{2}\right)}^{p-1}\|\tilde{\varphi}(\cdot, \cdot, 0)\|_{L^{4}\left(Q^{2}\right)}^{2} \\
& +\frac{p(p-1) \delta^{2}}{3 !}\|\tilde{v}(\cdot, \cdot, 0)\|_{L^{2(p-1)}\left(Q^{2}\right)}^{p-2}\|\tilde{\varphi}(\cdot, \cdot, 0)\|_{L^{\frac{6(p-1)}{p}}\left(Q^{2}\right)}^{\frac{1}{3}} \\
& +\frac{\epsilon \delta^{3}}{p+1}\|\tilde{\varphi}(\cdot, \cdot, 0)\|_{L^{3}\left(Q^{2}\right)}^{3},
\end{aligned}
$$

para um dado $\epsilon>0$.

Por outro lado, dado $\epsilon>0$ tome $\bar{\delta}>0$ tal que $P(\bar{\delta})<\epsilon$, onde

$$
\begin{aligned}
P(\bar{\delta}) & =\frac{p \bar{\delta}}{2 !}\|\tilde{v}(\cdot, \cdot, 0)\|_{L^{2(p-1)}\left(Q^{2}\right)}^{p-1}\|\tilde{\varphi}(\cdot, \cdot, 0)\|_{L^{4}\left(Q^{2}\right)}^{2} \\
& +\frac{p(p-1) \bar{\delta}^{2}}{3 !}\|\tilde{v}(\cdot, \cdot, 0)\|_{L^{2(p-1)}\left(Q^{2}\right)}^{p-2}\|\tilde{\varphi}(\cdot, \cdot, 0)\|_{L^{\frac{1}{3}}}^{\frac{6(p-1)}{p}}\left(Q^{2}\right) \\
& +\frac{\epsilon \bar{\delta}^{3}}{p+1}\|\tilde{v}(\cdot, \cdot, 0)\|_{L^{2}(p-1)\left(Q^{2}\right)} .
\end{aligned}
$$

Então, dado $\epsilon>0$ tome $\tilde{\delta}=\min \{\delta, \bar{\delta}\}$, logo

$$
|t|<\tilde{\delta} \Longrightarrow\left|\frac{g(\tilde{v}+t \tilde{\varphi})-g(\tilde{v})}{t}-<D g(\tilde{v}), \tilde{\varphi}>\right|<P(\tilde{\delta})<\epsilon,
$$

portanto, $g$ é $H_{q}$-diferenciável em $\tilde{v}$. 
Observe que podemos tomar $\bar{\delta}>0$ tal que $0<P(\bar{\delta})<\epsilon$, pois

$$
P(\bar{\delta})-\frac{\epsilon}{2} \stackrel{\bar{\delta} \rightarrow \infty}{\longrightarrow} \infty \quad \text { e } P(0)-\frac{\epsilon}{2}=-\frac{\epsilon}{2} .
$$

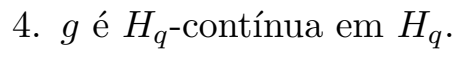

De fato, seja $(\tilde{w}, \tilde{\varphi}) \in H_{q} \times H_{q}$.

Analogamente ao caso anterior, usaremos a Fórmula de Taylor Infinitesimal.

$$
\begin{aligned}
|g(\tilde{w}+t \tilde{\varphi})-g(\tilde{w})| & \leq|t| \int_{\Omega_{q}}|\tilde{w}(x, y, 0)|^{p}|\tilde{\varphi}(x, y, 0)| d x d y \\
& +\frac{p|t|^{2}}{2 !} \int_{\Omega_{q}}|\tilde{w}(x, y, 0)|^{p-1}|\tilde{\varphi}(x, y, 0)|^{2} d x d y \\
& +\frac{p(p-1)|t|^{3}}{3 !} \int_{\Omega_{q}}|\tilde{w}(x, y, 0)|^{p-2}|\tilde{\varphi}(x, y, 0)|^{3} d x d y \\
& +\frac{1}{p+1} \int_{\Omega_{q}}\left|r_{3}(t \tilde{\varphi}(x, y, 0))\right| d x d y .
\end{aligned}
$$

Como,

$$
H_{p e r}^{\frac{1}{2}}\left(\mathbb{R}^{2}\right) \hookrightarrow L^{\frac{4 p}{3}}\left(Q^{2}\right) \quad \text { e } \quad H_{\text {per }}^{\frac{1}{2}}\left(\mathbb{R}^{2}\right) \hookrightarrow L^{4}\left(Q^{2}\right)
$$

temos que $(\tilde{w}(\cdot, \cdot, 0))^{p} \in L^{\frac{4}{3}}\left(Q^{2}\right)$ e $\tilde{\varphi}(\cdot, \cdot, 0) \in L^{4}\left(Q^{2}\right)$.

Então, pela Desigualdade de Hölder e a mesma idéia que usamos no item anterior, temos que dado $\epsilon>0$, existe $\delta>0$, onde $\widehat{P}(\delta)<\epsilon$, tal que

$$
|t|<\delta \Longrightarrow|g(\tilde{w}+t \tilde{\varphi})-g(\tilde{w})|<\widehat{P}(\delta)<\epsilon
$$

onde

$$
\begin{aligned}
\widehat{P}(\delta) & =\delta\|\tilde{w}(\cdot, \cdot, 0)\|_{L^{\frac{4 p}{3}}\left(Q^{2}\right)}^{p}\|\tilde{\varphi}(\cdot, \cdot, 0)\|_{L^{4}\left(Q^{2}\right)} \\
& +\frac{p \delta^{2}}{2 !}\|\tilde{w}(\cdot, \cdot, 0)\|_{L^{2(p-1)}\left(Q^{2}\right)}^{p-1}\|\tilde{\varphi}(\cdot, \cdot, 0)\|_{L^{4}\left(Q^{2}\right)}^{2} \\
& +\left(\frac{p(p-1)}{3 !}\|\tilde{w}(\cdot, \cdot, 0)\|_{L^{2(p-1)}\left(Q^{2}\right)}^{p-2}\|\tilde{\varphi}(\cdot, \cdot, 0)\|_{L^{\frac{6(p-1)}{p}}\left(Q^{2}\right)}^{\frac{1}{3}}\right. \\
& \left.+\frac{\epsilon}{p+1}\|\tilde{\varphi}(\cdot, \cdot, 0)\|_{L^{3}\left(Q^{2}\right)}^{3}\right) \delta^{3}
\end{aligned}
$$

Portanto, temos que $g$ é $H_{q}$-contínua em $H_{q}$.

5. $D g(\tilde{v}) \neq 0$.

De fato, suponha que $D g(\tilde{v})=0$, ou seja, para qualquer $\tilde{\varphi} \in H_{q}$, temos que

$$
<D g(\tilde{v}), \tilde{\varphi}>=0
$$


Em particular, para $\tilde{\varphi}=\tilde{v} \in H_{q}$,

$$
0=<D g(\tilde{v}), \tilde{v}>=\int_{\Omega_{q}}(\tilde{v}(x, y, 0))^{p+1} d x d y=a
$$

absurdo, pois $a \neq 0$. Portanto, $D g(\tilde{v}) \neq 0$.

Enfim, pelo Teorema do Multiplicador de Lagrange, temos que existe $\lambda \in \mathbb{R}$, o qual depende de $a$, tal que

$$
\int_{0}^{\infty} \int_{\Omega_{q}} \nabla \tilde{v} \nabla \tilde{\varphi} d x d y d z=\lambda \int_{\Omega_{q}}(\tilde{v}(x, y, 0))^{p} \tilde{\varphi}(x, y, 0) d x d y
$$

para qualquer $\tilde{\varphi} \in H_{q}$.

Para $\tilde{w}=\lambda^{-\frac{1}{1-p}} \tilde{v} \in H_{q}$, temos que

$$
\int_{0}^{\infty} \int_{\Omega_{q}} \nabla \tilde{w} \nabla \tilde{\varphi} d x d y d z=\int_{\Omega_{q}}(\tilde{w}(x, y, 0))^{p} \tilde{\varphi}(x, y, 0) d x d y
$$

para qualquer $\tilde{\varphi} \in H_{q}$. Portanto, o problema não linear (5.1) admite solução fraca.

Vejamos que a solução $\tilde{w}$ encontrada é não nula. Suponha $\tilde{w}=0$, então temos que $\lambda=0$, já que $\tilde{v}$ é uma função não nula, pelo fato de que

$$
\int_{\Omega_{q}}(\tilde{v}(x, y, 0))^{p+1} d x d y=a \neq 0
$$

Logo,

$$
\int_{0}^{\infty} \int_{\Omega_{q}} \nabla \tilde{v} \nabla \tilde{\varphi} d x d y d z=0
$$

para qualquer $\tilde{\varphi} \in H_{q}$. Em particular para $\tilde{\varphi}=\tilde{v} \in H_{q}$, temos que

$$
\begin{aligned}
\int_{0}^{\infty} \int_{\Omega_{q}}|\nabla \tilde{v}|^{2} d x d y d z=0 & \Longrightarrow \nabla \tilde{v}=0 \text { quase sempre } \\
& \Longrightarrow \tilde{v}=C \text { quase sempre }
\end{aligned}
$$

onde $C$ é uma constante não nula.

Mas para qualquer $z \geq 0$, temos que

$$
0=\int_{\Omega_{q}} \tilde{v}(x, y, z) d x=\operatorname{med}\left(\Omega_{q}\right) C \Longrightarrow C=0,
$$

o que é absurdo. Portanto, o problema não linear (5.1) admite solução fraca não nula. 


\section{Apêndice A}

\section{Apêndice}

\section{A.1 Funções Especiais}

Enunciaremos algumas proposições sobre extensões periódicas, pares e ímpares de funções, bem como propriedades de algumas funções especiais.

Proposição 13. ([19]) Seja $f \in C([0, l])$ e suponha que $f(0)=f(l)=0$. Então, a função $\tilde{f} d a d a$ por

$$
\tilde{f}(x)=\left\{\begin{array}{ccccc}
f(x-2 k l) & \text { se } & 2 k l & \leq x \leq & (2 k+1) l \\
-f(-x+2 k l) & \text { se } & (2 k-1) l<x & <k l
\end{array}\right.
$$

onde $k \in \mathbb{Z}$, é a extensão ímpar e 2l-periódica da função $f$ e é contínua em $\mathbb{R}$. Além disso, se $f \in C^{1}([0, l])$, então $\tilde{f} \in C^{1}(\mathbb{R})$ e $\tilde{f}_{x}$ é a extensão par e $2 l$-periódica da função $f_{x} \in C([0, l])$.

Proposição 14. ( [19]) Seja $u \in C([0, l])$. Então, a função $\tilde{u}$ dada por

$$
\tilde{u}(x)=\left\{\begin{array}{cccc}
u(x-2 k l) & \text { se } & 2 k l \leq x \leq & \leq k+1) l \\
u(-x+2 k l) & \text { se } & (2 k-1) l<x<
\end{array},\right.
$$

onde $k \in \mathbb{Z}$, é a extensão par e 2 -periódica da função u e é contínua em $\mathbb{R}$. Além disso, se $u \in C^{1}([0, l])$, então $\tilde{u} \in C^{1}([0, l])$ se, e somente se, $u_{x}(0)=u_{x}(l)=0$. Neste caso $\tilde{u}_{x}$ é a extensão impar e $2 l$-periódica da função $u_{x} \in C([0, l])$.

Definição 19. ([11]) Uma função $f: \mathbb{R}^{n} \longrightarrow \mathbb{R}$ é chamada de convexa se

$$
f(\tau x+(1-\tau) y) \leq \tau f(x)+(1-\tau) f(y)
$$

para qualquer $x, y \in \mathbb{R}^{n}$ e cada $0 \leq \tau \leq 1$.

Teorema 13. ([11]) Seja $f: \mathbb{R}^{n} \longrightarrow \mathbb{R}$ uma função convexa. Então, para cada $x \in \mathbb{R}^{n}$, existe $r \in \mathbb{R}^{n}$ tal que

$$
f(y) \geq f(x)+r \cdot(y-x)
$$

para qualquer $y \in \mathbb{R}^{n}$. Se $f$ for diferenciável em $x$, então $r=D f(x)$.

Observação 7. Temos também que se $f$ é $C^{2}$, então $f$ é convexa se, e somente se, $D^{2} f \geq 0$. 
Vejamos algumas informações importantes sobre as Funções de Bessel. Para um estudo mais detalhado ver [32], [34].

Seja $p \in \mathbb{R}$, a função de Bessel de primeiro tipo de ordem $p$ é dada por

$$
J_{p}(x)=\sum_{m=0}^{\infty} \frac{(-1)^{m}}{\Gamma(m+1) \Gamma(p+m+1)}\left(\frac{x}{2}\right)^{2 m+p} .
$$

Em particular, para $p=k \in \mathbb{Z}_{+}$, temos que

$$
J_{k}(x)=\sum_{m=0}^{\infty} \frac{(-1)^{m}}{m !(k+m) !}\left(\frac{x}{2}\right)^{2 m+p} .
$$

A função de Bessel de segundo tipo de ordem $p$, onde $p$ é fracionário, é dada por

$$
Y_{p}(x)=\frac{J_{p}(x) \cos (p \pi)-J_{-p}(x)}{\sin (p \pi)},
$$

em particular, para $p=n \in \mathbb{Z}$, temos que

$$
\begin{aligned}
Y_{n}(x) & =\frac{2}{\pi} J_{n}(x)\left(\ln \frac{x}{2}+C\right)-\frac{1}{\pi} \sum_{m=0}^{n-1} \frac{(n-m-1) !}{m !}\left(\frac{x}{2}\right)^{2 m-n} \\
& -\frac{1}{\pi} \sum_{m=0}^{\infty} \frac{(-1)^{m}}{m !(n+m) !}\left(\frac{x}{2}\right)^{2 m+n}\left(\sum_{k=1}^{n+m} \frac{1}{k}+\sum_{k=1}^{m} \frac{1}{k}\right)
\end{aligned}
$$

onde $C=0,57721 \ldots$ é chamada de constante de Euler.

Com isso temos para $p \in \mathbb{Z}$, que a combinação linear de $J_{p}$ e $Y_{p}$ é solução geral da equação de Bessel dada por

$$
x^{2} y^{\prime \prime}+x y^{\prime}+\left(x^{2}-p^{2}\right) y=0 .
$$

Lema 14. Seja $p \in \mathbb{R}$. Então,
1. $\frac{d}{d x}\left(x^{p} J_{p}(x)\right)=x^{p} J_{p-1}(x)$.
2. $\frac{d}{d x}\left(x^{-p} J_{p}(x)\right)=-x^{-p} J_{p+1}(x)$.
3. $x J_{p}^{\prime}(x)+p J_{p}(x)=x J_{p-1}(x)$.
4. $x J_{p}^{\prime}(x)-p J_{p}(x)=-x J_{p+1}(x)$.
5. $J_{p-1}(x)-J_{p+1}(x)=2 J_{p}^{\prime}(x)$.
6. $J_{p-1}(x)+J_{p+1}(x)=\frac{2 p}{x} J_{p}(x)$.

O próximo lema, nos revela o comportamento assintótico das funções de Bessel, para ordens e argumentos grandes. Para isto, consideraremos a seguinte notação

$$
f(x) \approx g(x) \text { quando } x \rightarrow a \text { se } \lim _{x \rightarrow a} \frac{f(x)}{g(x)}=1
$$


Lema 15. (Fórmula Assintótica - [5, 29])

1. (Comportameno Assintótico para ordens grandes)

$$
J_{\nu}(x) \approx \frac{1}{\sqrt{2 \pi \nu}}\left(\frac{e x}{2 \nu}\right)^{\nu}
$$

quando $\nu \rightarrow \infty$ e $x$ está fixado.

2. (Comportamento Assintótico para argumentos grandes)

$$
J_{n}(x) \approx\left(\frac{2}{\pi x}\right)^{\frac{1}{2}} \cos \left(x-\left(n+\frac{1}{2}\right) \frac{\pi}{2}\right),
$$

quando $x \rightarrow \infty$ e $n$ está fixado.

As funções de Bessel e suas derivadas possuem infinitos zeros positivos, os quais denotaremos, respectivamente, por $\sigma_{j p}$ e $\mu_{j p}, \operatorname{com} j=1,2, \cdots$.

Lema 16. ([1]) Os zeros das funções de Bessel e de suas derivadas satisfazem

1.

$$
\sigma_{1 p}<\sigma_{1(p+1)}<\sigma_{2 p}<\sigma_{2(p+1)}<\sigma_{3 p}<\cdots
$$

para qualquer $p \in \mathbb{Z}_{+}$.

2.

$$
p \leq \mu_{1 p}<\sigma_{1 p}<\mu_{2 p}<\sigma_{2 p}<\mu_{3 p}<\cdots
$$

para qualquer $p \in \mathbb{Z}_{+}$.

O zeros das funções de Bessel satisfazem uma estimativa dada no lema a seguir.

Lema 17. ( [25])

$$
\sigma_{j p}>\left(\left(j-\frac{1}{4}\right)^{2} \pi^{2}+p^{2}\right)^{\frac{1}{2}}
$$

para qualquer $p \geq 0$.

Lema 18. Considere as funções de Bessel $J_{p}$ e suas derivadas para $p \in \mathbb{R}$.

1. Se $\sigma, \mu$ dois zeros distintos de $J_{p}$, então

$$
\int_{0}^{\pi} x J_{p}\left(\frac{\sigma x}{\pi}\right) J_{p}\left(\frac{\mu x}{\pi}\right) d x=0
$$

O mesmo vale se $\sigma, \mu$ forem dois zeros distintos de $J_{p}^{\prime}$.

2.

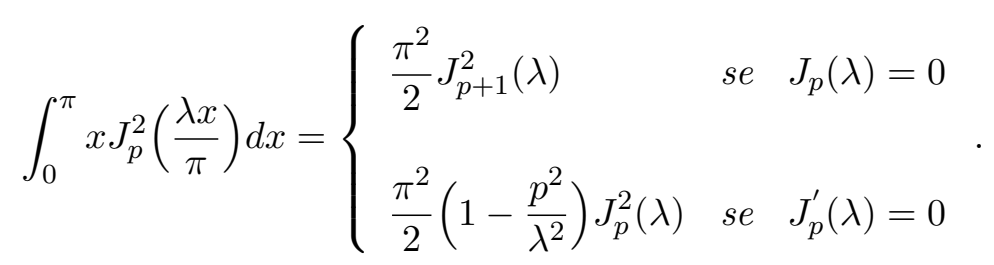


E por fim, temos que dada $f:[0, \pi] \longrightarrow \mathbb{R}$ uma função absolutamente integrável em $[0, \pi]$, sua série de Fourier-Bessel (de primeiro ou segundo tipo) é dada por

$$
c_{1} J_{p}\left(\frac{\lambda_{1 p} x}{\pi}\right)+c_{2} J_{p}\left(\frac{\lambda_{2 p} x}{\pi}\right)+\cdots+c_{j} J_{p}\left(\frac{\lambda_{j p} x}{\pi}\right)+\cdots,
$$

onde

$$
c_{j}=\frac{2}{\pi^{2} J_{p+1}^{2}\left(\lambda_{j p}\right)} \int_{0}^{\pi} x f(x) J_{p}\left(\frac{\lambda_{j p} x}{\pi}\right) d x
$$

quando $J_{p}\left(\lambda_{j p}\right)=0$, para qualquer $j=1,2, \cdots$, neste caso temos a série de Fourier-Bessel de primeiro tipo e

$$
c_{j}=\frac{2 \lambda_{j p}^{2}}{\pi^{2}\left(\lambda_{j p}^{2}-p^{2}\right) J_{p}^{2}\left(\lambda_{j p}\right)} \int_{0}^{\pi} x f(x) J_{p}\left(\frac{\lambda_{j p} x}{\pi}\right) d x,
$$

quando $J_{p}^{\prime}\left(\lambda_{j p}\right)=0$, para qualquer $j=1,2, \cdots$, chamada série de Fourier-Bessel de segundo tipo.

\section{A.2 Operadores}

Vejamos algumas definições, teoremas de operadores setoriais e potência fracionária de operadores.

Definição 20. ( [9]) Seja $0<\phi<\frac{\pi}{2}, M \geq 1$ e a $\in \mathbb{R}$. Dizemos que um operador $A: D(A) \subset H \longrightarrow H$ é setorial se

1. A é um operador fechado, densamente definido.

2. O conjunto resolvente, $\rho(A)$, contém o setor

$$
S_{a, \phi}=\{\lambda \in \mathbb{C}|\phi \leq| \arg (\lambda-a) \mid \leq \pi, \lambda \neq a\}
$$

3. Vale a estimativa

$$
\left\|(\lambda I-A)^{-1}\right\| \leq \frac{M}{|\lambda-a|}
$$

para qualquer $\lambda \in S_{a, \phi}$.

Definição 21. ([9]) Dizemos que um operador $A: D(A) \subset H \longrightarrow H$ é positivo do tipo $M \geq 1$ se

1. A é um operador fechado, densamente definido.

2. $\mathbb{R}_{+} \subset \rho(-A)$.

3. Existe $M \geq 1$ tal que

$$
(1+s)\left\|(s I+A)^{-1}\right\| \leq M
$$

para qualquer $s \in \mathbb{R}_{+}$.

Teorema 14. ([9]) Seja $A: D(A) \subset H \longrightarrow H$ um operador auto adjunto em um espaço de Hilbert $H$ com produto escalar $\langle\cdot, \cdot>$ e norma $\|\cdot\|$. Se A é limitado inferiormente, isto é, existe $m \in \mathbb{R}$ tal que

$$
<A(x), x>\geq m\|x\|^{2}
$$

para qualquer $x \in D(A)$, então $A$ é um operador setorial tal que $\operatorname{Re}(\sigma(A)) \geq m$. Em particular, um operador auto adjunto positivo definido é um operador setorial positivo. 
Teorema 15. ([9]) Seja $A: D(A) \subset H \longrightarrow H$ um operador positivo.

1. Para $z \in \mathbb{C}$ tal que $\operatorname{Re}(z) \geq 0, A^{z}$ é um operador fechado em $H$.

2. Se $z_{1}, z_{2} \in \mathbb{C}$ são tais que $\operatorname{Re}\left(z_{1}\right)>\operatorname{Re}\left(z_{2}\right)>0$, então $D\left(A^{z_{1}}\right) \subset D\left(A^{z_{2}}\right) \subset H$.

3. Para qualquer $n \in \mathbb{N}$ a potência $A^{n}$ coincide com o produto $A A \cdots A$.

4. Se $z \in \mathbb{C}$ tal que $\operatorname{Re}(z) \geq 0$, então $\overline{D\left(A^{z}\right)}=H$.

5. Se $z_{1}, z_{2} \in \mathbb{C}$ tais que $\operatorname{Re}\left(z_{1}\right), \operatorname{Re}\left(z_{2}\right), \operatorname{Re}\left(z_{1}+z_{2}\right) \neq 0$ ou $z_{1}, z_{2} \in \mathbb{R}$, então

$$
A^{z_{1}+z_{2}}(x)=A^{z_{1}} A^{z_{2}}(x)=A^{z_{2}} A^{z_{1}}(x)
$$

para $x \in D\left(A^{w}\right)$, onde $w \in\left\{z_{1}, z_{2}, z_{1}+z_{2}\right\}$ e $\operatorname{Re}(w)=\max \left\{\operatorname{Re}\left(z_{1}\right), \operatorname{Re}\left(z_{2}\right), \operatorname{Re}\left(z_{1}+z_{2}\right)\right\}$.

\section{A.3 Teorema do Multiplicador de Lagrange}

Antes de enunciarmos o Teorema do Multiplicador de Lagrange em espaços lineares topológicos, veremos algumas definições que estão em suas hipóteses.

Sejam $U$ um subconjunto aberto de um espaço vetorial topológico $E, G$ um subespaço linear de $E$ e uma função $f: U \longrightarrow \mathbb{R}$.

Definição 22. Dizemos que

1. fé G-contínua sobre $U$ se, e somente se,

$$
\lim _{t \rightarrow 0} f(x+t h)=f(x)
$$

para qualquer $(x, h) \in U \times G$.

2. $f$ é $G$-diferenciável em $x$ se, e somente se, existe uma aplicação linear $D f(x): G \longrightarrow \mathbb{R}$ tal que

$$
\lim _{t \rightarrow 0} \frac{f(x+t h)-f(x)}{t}=\operatorname{Df}(x)(h)
$$

para qualquer $h \in G$.

3. $f$ é fortemente G-diferenciável em $x$ se, e somente se, existe uma aplicação linear $D f(x): G \longrightarrow \mathbb{R}$ tal que

$$
\lim _{(s, t) \rightarrow 0} \frac{f(x+s t h+s k)-f(x)}{s}=D f(x)(k)
$$

para qualquer $h, k \in G$.

Lema 19. Sejam $U \subset E$ aberto, $E$ um espaço linear normado, $x \in U$ e $f: U \longrightarrow \mathbb{R}$ uma aplicação Fréchet Diferenciável em $x$. Então, $f$ é fortemente $G$-diferenciável em $x$ para qualquer subespaço linear $G$ de $E$. 
Teorema 16. (Teorema do Multiplicador de Lagrange em espaçoes lineares topológicos - [4]) Sejam $U$ um subconjunto aberto de um espaço vetorial topológico $E, G$ um subespaço linear de $E, x_{0} \in U$, $r \in \mathbb{R}$ e $f, g$ funções de $U$ em $\mathbb{R}$. Considere $M=\{x \in U \mid g(x)=r\}$. Assuma que

1. $f\left(x_{0}\right)$ é um mínimo (ou máximo) de $f(M)$.

2. $f$ é fortemente $G$-diferenciável em $x_{0}$ e g é G-diferenciável em $x_{0}$.

3. $g$ é $G$-contínua sobre $U$ e $D g\left(x_{0}\right) \neq 0$.

Então, existe $\lambda \in \mathbb{R}$ tal que

$$
D f\left(x_{0}\right)(h)=\lambda D g\left(x_{0}\right)(h),
$$

para qualquer $h \in G$. 


\section{Referências Bibliográficas}

[1] Abramowitz, M.; Stegun, I. A. Handbook of mathematical functions with formulas, graphs, and mathematical tables. New York: Dover Publications, 1965. 1046 p.

[2] Adams, R. A. Sobolev Spaces. New York: Academic Press, 1975. v.65. (Pure and applied mathematics series)

[3] Amann, H. Nonhomogeneous Linear and Quasilinear Elliptic and Parabolic Boundary Value Problems: in Function Spaces, Differential Operators and Nonlinear Analysis. Teubner, Stuttgart, Leipzig, 9-126, 1993.

[4] An, L. H.; Du, P. X.; Duc, D. M.; Tuoc P. V. Lagrange Multipliers for Functions Derivable along Directions in a Linear Subspace. Proceedings of the American Mathematical Society, v.133, n.2, p. 595-604, 2004.

[5] Bell, W. W. Special Funtions for Scientists and Engineers. Canada: D. Van Nostrand Company Ltda, 1970. 247 p.

[6] Brézis, H. Análisis functional Teoría y aplicaciones. Madrid: Alianza Editorial, S.A., 1984. 233 p.

[7] Cabré, X.; Tan, J. Positive solutions of nonlinear problems involving the square root of the Laplacian. Advances in Mathematics, v.224, p. 2052-2093, 2010.

[8] Caffarelli, L.; Silvestre, L. An extension problem related to the fractional Laplacian. Comm. in Partial Differential Equations, v.32, p. 1245-1260, 2007.

[9] Czaja, R. Differential Equations with Sectorial Operator. Katowice: Wydawnictwo Uniwersytetu Slaskiego, 2002. 120 p.

[10] Engel, K. J.; Nagel, R. A Short Course on Operator Semigroups. New York: Springer Science, Business Media, LLC, 2006. 256 p.

[11] Evans, L. C. Partial Differential Equations. United States of America: American Mathematical Society, 1998. v.19.

[12] Figueiredo, D. G. Análise de fourier e equações diferenciais parciais. Rio de Janeiro: IMPA, 2003. 274 p. (Projeto Euclides)

[13] Figueiredo, D. G. Análise I. Rio de Janeiro: LTC, 1996. 256 p. 
[14] Henrot, A. Extremum problems for eigenvalues of elliptic operators. Berlin: Birkhäuser Verlag, 2006. 202 p.

[15] Henry, D. Geometric Theory of Semilinear Parabolic Equations. Berlin: Springer-Verlag, 1981. 348 p.

[16] Hormander, L. Linear Partial Differential Operators. Berlin: Springer, 1969. 285 p.

[17] Iório R.; Iório, V. M. Fourier Analysis and Partial Differential Equations. New York: Cambridge University Press, 2001. 411 p.

[18] Iório R.; Iório, V. M. Equações diferenciais parcias: uma introdução. Rio de Janeiro: IMPACNPQ, 1988. 366 p. (Projeto Euclides)

[19] Iório, V. EDP, um curso de graduação. Rio de Janeiro: IMPA, 2005. 246 p. (Coleção matemática universitária)

[20] Kozlov, V. A.; Maz'ya, V. G.; Rossmann, J. Elliptic Boundary value problems in domains with point singularities. Providence, R. I.: American Mathematical Society, 1997. 414 p. (Mathematical surveys and monographs)

[21] Kreyszig, E. Introductory Functional Analysis with Applications. New York: John Wiley \& Sons, 1989. 688 p.

[22] Kufner, A.; John, O.; Fuécík, S. Function Spaces. Leyden: Noordhoff International Publishing, 1977. $454 \mathrm{p}$.

[23] Lima, E. L. Curso de Análise, Volume 1. Rio de Janeiro: IMPA, 2006. 431 p. (Projeto Euclides)

[24] Lima, E. L. Curso de Análise, Volume 2. Rio de Janeiro: IMPA, 2000. 557 p. (Projeto Euclides)

[25] Maccann, R. C. Lower Bounds for the Zeros of Bessel Functions. Proceedings of the American Mathematical Society, v.64, n.1, p. 101-103, 1977.

[26] Melo, R. A. A Teoria de Semigrupo Aplicada às Equações Diferenciais Parciais. 2006. $183 f$. Dissertação (Mestrado em Matemática) - Centro de Ciências e Tecnologia, Universidade Federal de Campina Grande, Campina Grande. 2006.

[27] Naylor, A. W.; Sell, G. R. Linear operator theory in engineering and science. New York: SpringerVerlag, 1982. $624 \mathrm{p}$.

[28] Pazy, A. Semigroups of Linear Operators and Applications to Partial Differential Equations. New York: Springer-Verlag, 1983. 279 p.

[29] Polyanin, A. D. Handbook of Linear Partial Differential Equations for Engineers and Scientists. Boca Raton: Chapman \& Hall/CRC, 2002. 781 p.

[30] Protter, M. H.; Morrey, C. B. Intermediate Calculus. New York: Springer-Verlag, 1985. 555 p. 
[31] Rabinowitz, P. H. Minimax methods in critical point theory with applications to differential equations. Providence, R. I.: American Mathematical Society, 1986. (Regional conference series in mathematics)

[32] Tolstov, G. P. Fourier Series. New Jersey: Prentice-Hall, 1962. 336 p.

[33] Triebel, H. Interpolation theory, function spaces, differential operators. Amsterdam: NorthHolland Publishing Company, 1978. 528 p.

[34] Watson, G. N. A Treatise on the Theory of Bessel Functions. New York: Cambridge University Press, 1945. 804 p. 


\section{Índice Remissivo}

cone, 9

domínio tipo, 10

equação

Bessel, 100

espaço

Sobolev das funções periódicas, 15

das distribuições temperadas, 10

Hölder, 12

Schwartz, 10

Slobodeckij em $\mathbb{R}^{n}, 10$

Slobodeckij em $U, 11$

Sobolev em $\mathbb{R}^{n}, 10$

Sobolev em $U, 11$

fórmula assintótica, 101

função

G-contínua, 103

$G$-diferenciável, 103

Bessel

primeiro tipo, 100

segundo tipo, 100

convexa, 99

fortemente $G$-diferenciável, 103

imersão

compacta, 13

contínua, 12

operador

limitado inferiormente, 24

positivo, 102

setorial, 102

traço, 19

série

Fourier-Bessel, 102

teorema

Multiplicador de Lagrange, 104 